Order Number 9507759

Neurological evidence in support of a specialized phonetic processing module

Gokcen, Jeanne Marie, Ph.D.

The Ohio State University, 1992

Copyright @1992 by Gokcen, Jeanne Marie. All rights reserved.

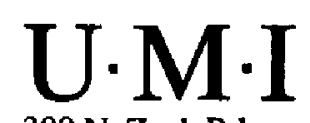

300 N. Zeeb Rd.

Ann Arbor, MI 48106 


\title{
NEUROLOGICAL EVIDENCE IN SUPPORT OF A SPECIALIZED PHONETIC PROCESSING MODULE
}

\section{DISSERTATION}

\author{
Presented in Partial Fulfillment of the Requirements for \\ the Degree Doctor of Philosophy in the Graduate \\ School of the Ohio State University
}

By

Jeanne Marie Gokcen, B. S., M. A.

标虫中

The Ohio State University

1992

Dissertation Committee:

Approved by

Robert A. Fox

Michael W. Torello

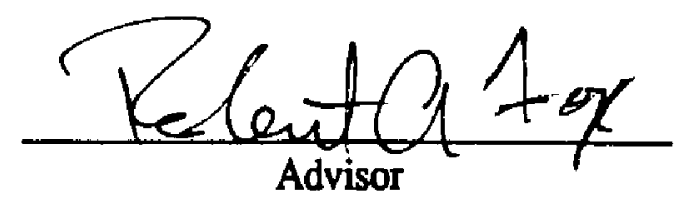

Lida G. Wall

Division of Speech and Hearing Science 
Copyright by

Jeanne Marie Gokcen

1992 
This dissertation is lovingly dedicated to my husband, Sedat, to my daughter, Ajda,

and to my parents, Albert and Rita Holloway. 


\section{ACKNOWLEDGMENTS}

First, I would like to express my heartfelt appreciation to Dr. Robert A. Fox, without whose insistence I would not have come to attend the fine Speech Science program at The Ohio State University. In his capacity as my advisor and as the chair of my dissertation committee, Dr. Fox has continually provided invaluable guidance throughout my program and my research. In addition, he is responsible for my receipt of a number of awards at this institution, for which I am deeply grateful. I express sincere appreciation and admiration to Dr. Michael Torello, one of my committee members, for his insights and for his amazing ability to completely grasp concepts that are outside of his considerable area of expertise. I also thank Dr. Torello for his constant consideration of my program during a tumultuous period. I am grateful to Dr. Lida Wall for agreeing to become a member of my committee at a late date and for her suggestions for the manuscript. I would like to thank Dr. Amy Wohlert for providing me with guidance which initiated this project and for her comments on the manuscript. I value highly the friendships that I have developed with my committee members. Dr. Mary Beckman's observations about the manuscript are appreciated. Also, I thank Dr. Marlin Languis of Excellence in Learning, Inc. for the use of his equipment and his assistance in completing part of my analyses. 
I would like my daughter, Ajda, to know that her development, both before and after her birth, has provided unspeakable joy and motivation to me to complete this project. I thank my parents, Albert and Rita Holloway, for their encouragement since I was young to always strive to attain my highest goals and for their pride that I can feel from hundreds of miles away. Finally, and most importantly, I express my deepest love and gratitude to my husband, Sedat, for his unflagging faith in my ability to see this project through to its completion. He has been my inspiration, my sounding board, and has been a partner not only by being the "designated parent" more often than not, but by working by my side to help me finish this dissertation. I literally could not have done this without him. 


\section{VITA}

March 30, 1960

Born - Chicago, Illinois

1982

B. S., Ohio University, Athens, Ohio

1984

M. A., Ohio University, Athens, Ohio

1984-1985

Speech-Language Pathologist, InSpeech, Inc., Lima, Ohio

1985-1988

Speech-Language Pathologist, Children's Hospital, Columbus, Ohio

\section{PUBLICATIONS}

Fox, R. A., Wall, L. G., and Gokcen, J. (1992). Age-related differences in processing dynamic information to identify vowel quality. Journal of Speech and Hearing Research, 35, 892-902.

\section{FIELDS OF STUDY}

Major Field: Speech and Hearing Science Dr. Robert A. Fox, Advisor 
TABLE OF CONTENTS

DEDICATION .........................................................................................ii

ACKNOWLEDGEMENTS …............................................................ii

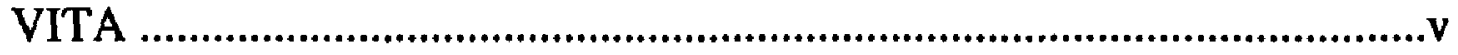

LIST OF TABLES ...................................................................................viii

LIST OF FIGURES ……………………...................................................

$\begin{array}{ll}\text { CHAPTER } & \text { PAGE }\end{array}$

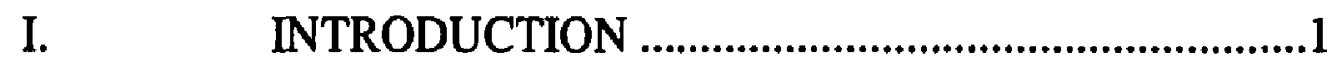

II. METHODOLOGY ...................................................4

III. RESULTS AND DISCUSSION .................................61

IV. CONCLUSIONS AND FUTURE DIRECTIONS ......90 


\section{APPENDICES}

A. Instructions given to subjects prior to each condition .96

B. Representative waveforms of three subjects for each stimulus within each condition

C. Grand average waveforms for each stimulus within each condition 125

BIBLIOGRAPHY 136 


\section{LIST OF TABLES}

TABLE

PAGE

1. Parameter values of an exemplar [da]

2. Parameter values of an exemplar [ga] .48

3. Mean latency values and standard deviations of

$\mathrm{N} 1$ across subjects in Conditions 1 and 2 68

4. Mean latency values and standard deviations of $\mathrm{P} 1$ across subjects in Conditions 1 and 2

5. Mean latency values and standard deviations of $\mathrm{N} 2$ across subjects in Conditions 1 and 2

6. Mean amplitude values and standard deviations of $\mathrm{N} 1$ across subjects in Conditions 1 and 2 ...........................72

7. Mean amplitude values and standard deviations of P1 across subjects in Conditions 1 and 2

8. Mean amplitude values and standard deviations of N2 across subjects in Conditions 1 and 2

9. Mean latency values and standard deviations of $\mathrm{N} 1$ across subjects in Conditions 3 and 4 .77

10. Mean latency values and standard deviations of $P 1$ across subjects in Conditions 3 and 4 .78 
11. Mean latency values and standard deviations of N2 across subjects in Conditions 3 and 4

12. Mean amplitude values and standard deviations of N1 across subjects in Conditions 3 and 4

13. Mean amplitude values and standard deviations of

P1 across subjects in Conditions 3 and 4

14. Mean amplitude values and standard deviations of

N2 across subjects in Conditions 3 and 4

15. Mean latency values and standard deviations of

$\mathrm{N} 1, \mathrm{P} 1$, and $\mathrm{N} 2$ across subjects in Condition 5

16. Mean amplitude values and standard deviations of $\mathrm{N} 1, \mathrm{P} 1$, and $\mathrm{N} 2$ across subjects in Condition 5 


\section{LIST OF FIGURES}

FIGURES

PAGE

1. A schematic spectrogram of [da] showing formant transitions.

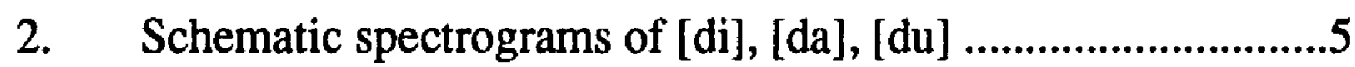

3. Schematic spectrograms of $[\mathrm{ba}]$, [da], [ga] ..........................6

4. Schematic representation of the syllables [da] and [ga] .........22

5. Schematic of ERP averaging process. Multiple trials are averaged together and background EEG not related to the stimulus cancels itself out, resulting in a waveform with more distinctive peaks

6. International 10-20 System of Electrode Placement. The figure provides the alphanumeric designations of electrode placement on the scalp for EEG recordings 54

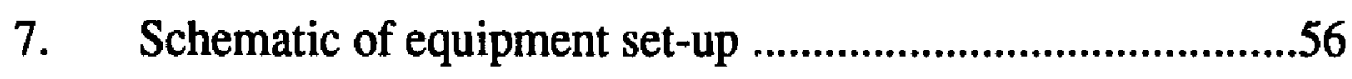

8. Sample waveform referencing significant measurement points used in this study

9. Sample waveform from Subject 4. This waveform represents only the responses to plain [da] in Condition 1 (plain [da] vs. duplex [da]) .63 
10. Sample waveform from Subject 4. This waveform represents only the responses to duplex [da] in Condition 1 (plain [da] vs. duplex [da])

11. Sample waveform from Subject 13. This waveform represents only the responses to plain [da] in Condition 1 (plain [da] vs. duplex [da])

12. Sample waveform from Subject 13. This waveform represents only the responses to duplex [da] in Condition 1 (plain [da] vs. duplex [da])

13. Sample waveform from Subject 4. This waveform represents only the responses to plain [ga] in Condition 2 (plain [ga] vs. duplex [ga])

14. Sample waveform from Subject 4. This waveform represents only the responses to duplex [ga] in Condition 2 (plain [ga] vs. duplex [ga])

15. Sample waveform from Subject 4. This waveform represents only the responses to duplex [da] in Condition 3 (duplex [da] vs. duplex [ga])

16. Sample waveform from Subject 4. This waveform represents only the responses to duplex [ga] in Condition 3 (duplex [da] vs. duplex [ga]

17. Sample waveform from Subject 4. This waveform represents only the responses to plain [da] in Condition 4 (plain [da] vs. plain [ga]).

18. Sample waveform from Subject 4. This waveform represents only the responses to plain [ga] in Condition 4 (plain [da] vs. plain [ga]) 
19. Sample waveform from Subject 4. This waveform represents only the responses to tone glide [da] in Condition 5 (tone glide [da] vs. tone glide [ga])

20. Sample waveform from Subject 4. This waveform represents only the responses to tone glide [ga] in Condition 5 (tone glide [da] vs. tone glide [ga]

21. Sample waveform from Subject 13. This waveform represents only the responses to plain [ga] in Condition 2 (plain [ga] vs. duplex [ga])

22. Sample waveform from Subject 13. This waveform represents only the responses to duplex [ga] in Condition 2 (plain [ga] vs. duplex [ga])

23. Sample waveform from Subject 13. This waveform represents only the responses to duplex [da] in Condition 3 (duplex [da] vs. duplex [ga])

24. Sample waveform from Subject 13. This waveform represents only the responses to duplex [ga] in Condition 3 (duplex [da] vs. duplex [ga])

25. Sample waveform from Subject 13. This waveform represents only the responses to plain [da] in Condition 4 (plain [da] vs. plain [ga])

26. Sample waveform from Subject 13. This waveform represents only the responses to plain [ga] in Condition 4 (plain [da] vs. plain [ga])

27. Sample waveform from Subject 13. This waveform represents only the responses to tone glide [da] in Condition 5 (tone glide [da] vs. tone glide [ga]) 
28. Sample waveform from Subject 13. This waveform represents only the responses to tone glide [ga] in Condition 5 (tone glide [da] vs. tone glide [ga])

29. Sample waveform from Subject 19. This waveform represents only the responses to plain [da] in Condition 1 (plain [da] vs. duplex [da])

30. Sample waveform from Subject 19. This waveform represents only the responses to duplex [da] in Condition 1 (plain [da] vs. duplex [da])

31. Sample waveform from Subject 19. This waveform represents only the responses to plain [ga] in Condition 2 (plain [ga] vs. duplex [ga])

32. Sample waveform from Subject 19. This waveform represents only the responses to duplex [ga] in Condition 2 (plain [ga] vs. duplex [ga])

33. Sample waveform from Subject 19. This waveform represents only the responses to duplex [da] in Condition 3 (duplex [da] vs. duplex [ga])

34. Sample waveform from Subject 19. This waveform represents only the responses to duplex [ga] in Condition 3 (duplex [da] vs. duplex [ga])

35. Sample waveform from Subject 19. This waveform represents only the responses to plain [da] in Condition 4 (plain [da] vs. plain [ga])

36. Sample waveform from Subject 19. This waveform represents only the responses to plain [ga] in Condition 4 (plain [da] vs. plain [ga]) 
37. Sample waveform from Subject 19. This waveform represents only the responses to tone glide [da] in Condition 5 (tone glide [da] vs. tone glide [ga])

38. Sample waveform from Subject 19. This waveform represents only the responses to tone glide [ga] in Condition 5 (tone glide [da] vs. tone glide [ga])

39. Grand average waveform across all subjects in Condition 1 (plain [da] vs. duplex [da]. This waveform represents only the responses to plain [da]

40. Grand average waveform across all subjects in Condition 1 (plain [da] vs. duplex [da]. This waveform represents only the responses to duplex [da]

41. Grand average waveform across all subjects in Condition 2 (plain [ga] vs. duplex [ga]). This waveform represents only the responses to plain [ga]

42. Grand average waveform across all subjects in Condition 2 (plain [ga] vs. duplex [ga]). This waveform represents only the responses to duplex [ga]

43. Grand average waveform across all subjects in Condition 3 (duplex [da] vs. duplex [ga]). This waveform represents only the responses to duplex [da]

44. Grand average waveform across all subjects in Condition 3 (duplex [da] vs. duplex [ga]). This waveform represents only the responses to duplex [ga]

45. Grand average waveform across all subjects in Condition 4 (plain [da] vs. plain [ga]). This waveform represents only the responses to plain [da] 
46. Grand average waveform across all subjects in Condition 4 (plain [da] vs. plain [ga]). This waveform represents only the responses to plain [ga]

47. Grand average waveform across all subjects in

Condition 5 (tone glide [da] vs. tone glide [ga]).

This waveform represents only the responses

to tone glide [da]

48. Grand average waveform across all subjects in Condition 5 (tone glide [da] vs. tone glide [ga]). This waveform represents only the responses to tone glide [ga] 


\section{CHAPTER I INTRODUCTION}

Human speech perception is a process which deceptively appears to be effortless because it occurs rapidly and unconsciously, while, in truth, it is so complex that it has defied complete explanation despite the efforts of scores of scientists over the course of many years. In attempts to answer the intriguing question of how humans perceive speech, scientists have postulated many theories of speech perception over which there has been, and continues to be, much controversy.

Any account of the speech perception process must contend with the inherent characteristics of the acoustic speech signal. These characteristics have been problematic because the acoustic cues signalling particular phonetic segments vary depending on the phonemes that surround it. Although instinctively it would seem that humans hear words that are separate from each other, in actuality it has been shown repeatedly that the speech signal is a continuous flow of sound that is acoustically complex and continuously varying, and it is difficult to distinguish segments and individual words given just the acoustic structure. Within their models of speech perception, theorists have tried to address the issues of invariance, linearity, segmentation, and units of perceptual analysis. 
Before the basic issues of speech perception are discussed, the characteristics of the acoustic signal should be described. In the majority of speech sounds the vibrations of the air in the passages of the mouth, throat, and nose (which are collectively known as the vocal tract) serve as the movements which initiate sound waves. In general the vocal tract is considered to be like a tube that is closed at one end by the vocal folds and open at the other end through the lips and nose; thus it forms a resonating chamber of a complex shape. The air in the vocal tract will vibrate, or resonate, in different ways when the articulators (teeth, lips, tongue, etc.) are in different positions (Ladefoged, 1962). Resonances of the vocal tract are known as formants. Formants are concentrations of energy around certain frequencies, depending on the shape of the vocal tract.

The acoustic signal can be represented visually on a spectrogram. On a spectrogram, it can be seen that a main aspect of the signal that varies from one phoneme to another is a pattern consisting of three formants that are numbered from low to high frequencies, known as F1 (the first formant), F2 (the second formant) and F3 (the third formant). As the articulators move from the physical configuration required for the production of one phoneme to that required for another (e. g., from $/ \mathrm{d} /$ to $/ \mathrm{a} /$ ), there is a change in frequency over time called a formant transition (Figure 1) (Pickett, 1980).

There are also many other characteristics of an acoustic signal that contribute to the distinction of different phonemes, such as voicing, frication noise, nasality, etc., but these will not be described here since the formants and the formant transitions are the characteristics which are manipulated in this dissertation. Somehow the acoustic signal represented by these formants 


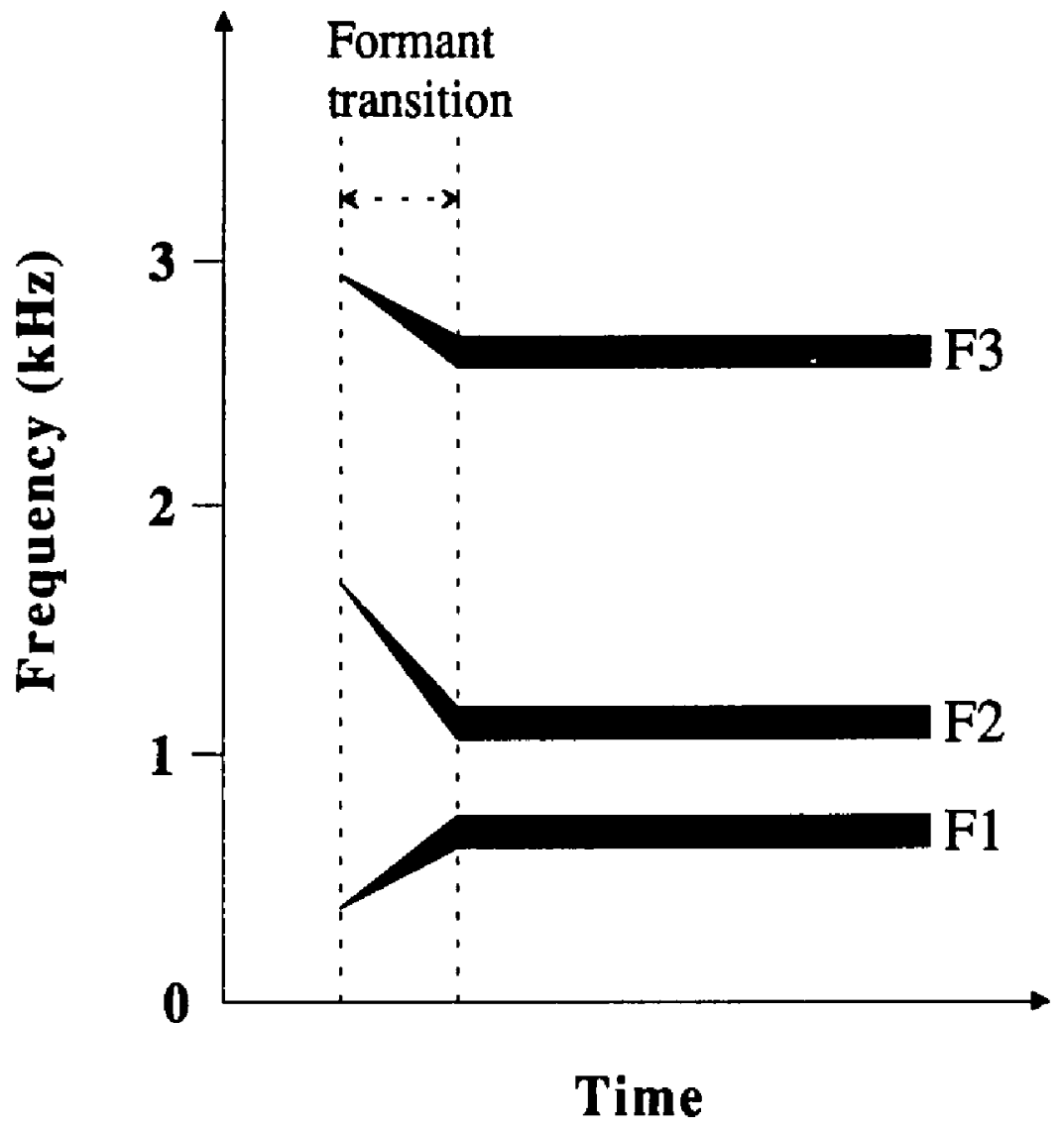

FIGURE 1. A schematic spectogram of [da] showing formant transitions. 
and formant transitions (and other acoustic characteristics) is converted by the listener into discrete linguistic units and then formed into words and sentences which convey the message intended by the speaker.

Now that the acoustic speech signal has been described, the problems involved in explaining the process of speech perception, that is, invariance, linearity, segmentation, and units of perceptual analysis, can be more clearly understood. The principle of invariance was presented by Chomsky and Miller in 1963. It states that for every phoneme $X$ there must be a set of features which uniquely identify that phoneme such that whenever that phoneme is present, those features must be present, and when that phoneme is absent, then so should those features. This principle seems to be violated by the acoustic signal because features and transitions from adjacent phonemes overlap, so that features from one phoneme can be observed in the signal of the preceding and following phonemes (which is known as coarticulation). This especially seems to be the case for vowels influencing the acoustic characteristics of consonants. For example, the three $/ d / s$ in the syllables [di], [da], and [du] display different formant transitions. The transition from $/ \mathrm{d} /$ to $/ \mathrm{i} /$ shows a rising frequency for both $\mathrm{F} 1$ and $\mathrm{F} 2$; from /d/ to /a/ the F1 transition rises moderately and the $F 2$ transition falls moderately; from $/ d$ / to $/ \mathrm{u} /$ the $\mathrm{F} 1$ transition rises slightly and the $\mathrm{F} 2$ transition falls sharply (Figure 2). The case is also true, however, of consonants influencing vowels, as in [ba], [da], and [ga] (Figure 3). The apparent variance of the acoustic signal makes it difficult to segment the signal into phonemes which are free of other phonemic influences. In 


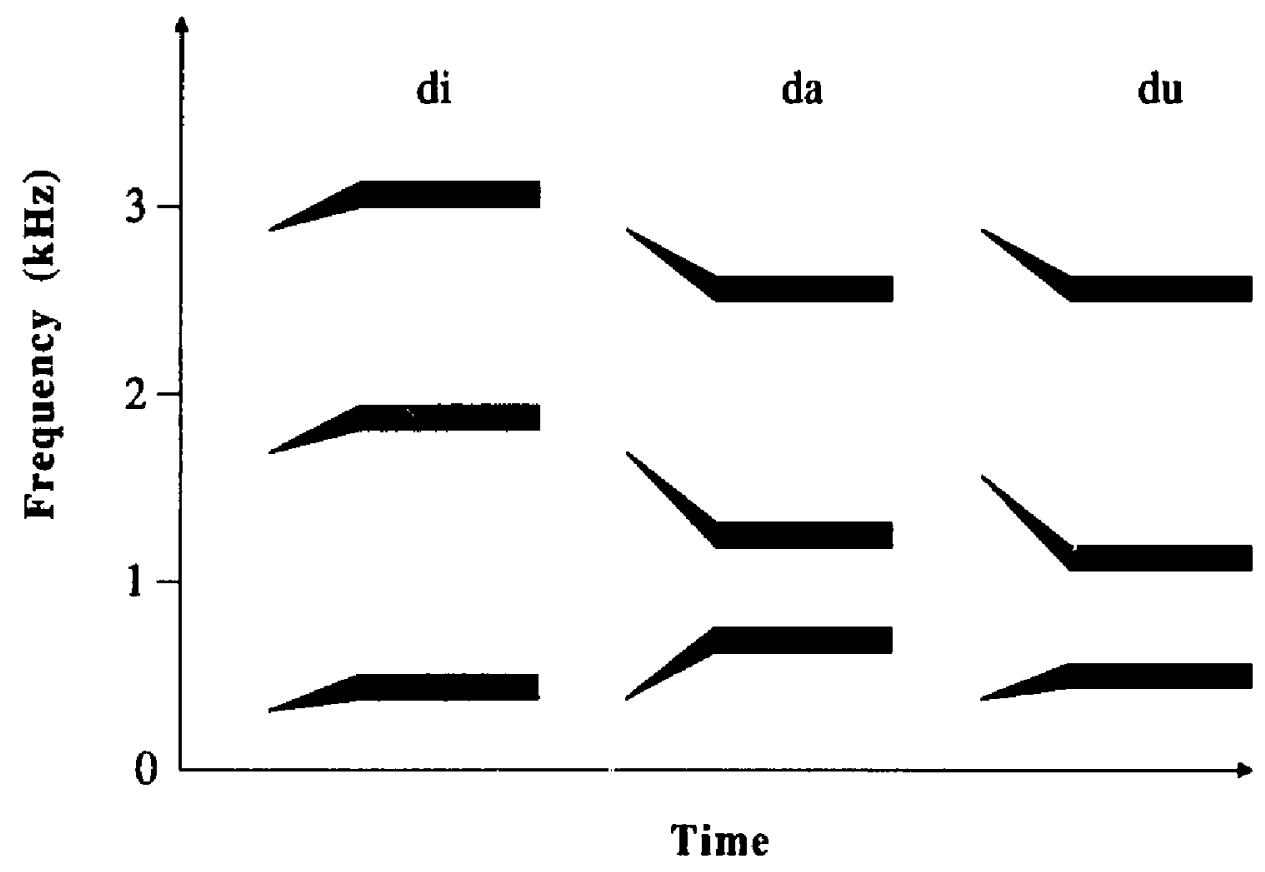

FIGURE 2. Schematic spectograms of [di], [da], [du]. 


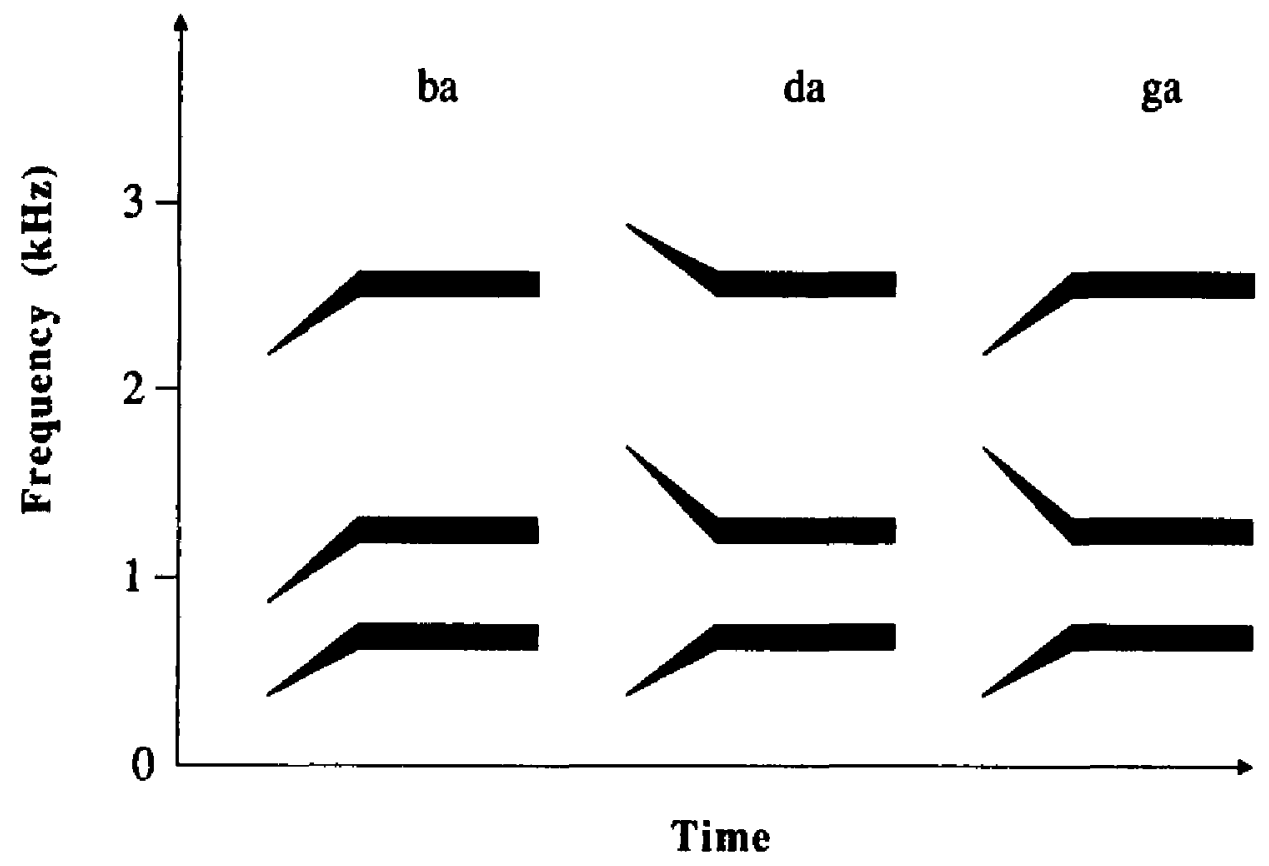

FIGURE 3. Schematic spectograms of [ba], [da], [ga]. 
addition, contextual influences are not limited to adjacent phonemes, but also can come from phonemes farther away in the word.

Another problem in dealing with the speech signal involves the issue of linearity. The linearity condition states that for each perceived phoneme there should be a unique corresponding stretch of sound in the utterance. Furthermore, if phoneme $\mathrm{X}$ is followed by phoneme $\mathrm{Y}$ in the phonemic representation, the portion of sound corresponding to phoneme $\mathrm{X}$ must precede the portion of sound corresponding to phoneme $\mathrm{Y}$ in the physical signal (Chomsky and Miller, 1963). As in the case of invariance mentioned above, because of coarticulation and other contextual effects, acoustic features for adjacent phonemes can be seen across other phonemes in the speech waveform. This results in stretches of the speech waveform in which acoustic features of more than one phoneme are present. Therefore, not only is there rarely a particular stretch of sound that corresponds uniquely to a given phoneme, it is also rare that the acoustic features of one phoneme always completely precede or follow the acoustic features of adjacent phonemes in the physical signal (Luce and Pisoni, 1987).

Because, as some would argue, the speech signal apparently violates the principles of invariance and linearity, it is extremely difficult to segment the speech signal into phonemes, syllables, and words. The issue of segmentation gives rise to the question of specifying the nature of the basic units of perceptual analysis, that is, what minimal segment of the speech signal is obligatory in the perceptual processing of speech? Various suggestions have been offered including the phonetic feature, the phoneme, the syllable, the word, context-sensitive allophones, and context-sensitive 
spectra (Cole and Scott, 1974; Studdert-Kennedy, 1974; Wicklegren, 1969; Klatt, 1980a), but conclusive evidence that singularly supports any one of these proposed units is lacking. There are data, however, suggesting that each of these separate units may be important at some level (Pisoni and Sawusch, 1975).

As mentioned previously, there are many theories of speech perception, too many to discuss all of them in any detail here. A representative sampling of these theories will be presented. First of all, there is a common view of speech perception that involves stages of analysis in converting the speech signal to the intended message (Fant, 1967; Studdert-Kennedy, 1974; Pisoni and Sawusch, 1975). In this view there are four proposed conceptual stages of analysis: (1) auditory, (2) phonetic, (3) phonological, and (4) lexical, syntactic, and semantic. Recently, a fifth stage has been added to this model (Kewley-Port, 1980; Kewley-Port and Luce, 1984; Searle, Jacobson, and Rayment, 1979, Luce and Pisoni, 1987), that of peripheral auditory processing, which is actually a subcomponent of the first stage (auditory analysis).

During peripheral auditory processing, the earliest transformations of the speech signal are performed by the peripheral auditory system, which include the physical components of the system such as the tympanic membrane, the ossicles, the cochlea, the hair cells, and the auditory nerve fibers. In the auditory stage, acoustic information about spectral structure, fundamental frequency, overall intensity, duration of the signal, and amplitude onsets and offsets is extracted and coded by the auditory system. In this stage an acoustic feature analysis is carried out which provides 
"cues" to phonetic classification. At the next level, the phonetic stage, acoustic features are mapped onto phonetic features such as voicing, tenseness, and nasalization. At the level of phonological analysis, the phonetic segments are converted into phonemes such as /b/ and /i/. After the phonological analysis, several higher order levels of analysis are carried out on the speech signal. During lexical analysis word recognition and lexical access occur. Then syntactic, semantic, and prosodic information is processed in order to interpret the utterance. The amount of interaction within and between each stage varies according to the particular speech perception model.

One model of speech perception that represents a highly interactive speech perception model is the TRACE model by Elman and McClelland (1986). In the TRACE model initial peripheral auditory processing leads to a neural spectrogram, which is a mental picture of the speech signal that provides information about the changing frequencies and intensities over time. There are three levels of processing with associated layers of nodes for: (1) feature detection; (2) phoneme detection; and (3) word detection. Within each level are many processing units, each of which stands for a hypothesis about a particular perceptual object occurring at a particular point in time defined relative to the beginning of the spoken signal. At the feature level are several banks of feature detectors, one for each of several dimensions of speech sounds. At the phoneme level are detectors for each of the phonemes. At the word level are detectors for each word. These levels form a completely interconnected network in which any level of processing can influence any other level of processing at any time. This 
model thus allows for both bottom-up and top-down processing.

Processing at any of the levels can occur simultaneously, indicating a parallel distributed processing system. Influences among the neurons can be excitatory or inhibitory, but with some constraints. Interconnections between levels can be excitatory only, so feature detectors can excite phoneme detectors or word detectors and vice versa, but not inhibit them. Interconnections within levels are inhibitory only, so if the detector for the phoneme $/ \mathrm{b} /$ is highly activated, it can inhibit the detector for the phoneme /s/, but cannot inhibit any feature detectors. Information from the initial peripheral auditory analysis is available for processing at all the levels and pertinent nodes are activated. As more sensory information becomes available, the detectors with stronger activation will inhibit weaker detectors within their level. Eventually, the word node with the highest activation will be recognized as the intended word. The entire network of units is called "the Trace," because the pattern of activation left by a spoken input is a trace of the analysis of the input at each of the three processing levels. This Trace is dynamic, since it consists of activations of processing elements, and these processing elements continue to interact as time goes on. The TRACE model processes information from all levels of the incoming acoustic input; it therefore claims to have no one basic unit of perception. TRACE accounts for contextual effects by allowing input from all sources.

Another speech perception theory is the cohort theory offered by Marslen-Wilson (1980). In the cohort theory there are two stages of processing and the basic unit of processing is the feature. The first stage is autonomous and acoustic information at the beginning of the word (e. g., the 
initial consonant cluster and vowel) activates all the nodes representing words which begin with these same letters. All of these words form the "cohort" for the input word. The second stage is interactive and allows all possible sources of knowledge (e. g., syntactic, semantic, etc.) to influence the selection of a cohort. As acoustic information continues to arrive and information from other sources narrow the possibilities, cohort members that no longer fit do not receive any further activation and so they decay. When only a single cohort member remains, then that word is recognized as the correct word. The cohort theory accounts for contextual effects by allowing influence from syntactic and semantic sources, which can contribute to the activation of a cohort. If no words remain in the cohort, the target stimulus is considered a nonword.

Klatt's (1980a) Lexical Access from Spectra (LAFS) model assumes direct, noninteractive access of lexical entries based on context-sensitive spectral sections. Adults store a spectral representation of all lawful diphone (i. e., two-phoneme) sequences which overcome much contextual variability because both the spectral characteristics of the phonemes and the transitions from one phoneme to another are stored. The listener computes spectral representations of an input word and compares them with the spectral representations stored in memory. Word recognition occurs when a best match is found between the input spectra and the diphone representations. Typically, word recognition is direct with no levels involving segments.

Each theory presented above has generated interest among speech perception theorists, and they have had at least some influence in the manner 
in which the process of speech perception is viewed; however, an extremely influential theory is the Motor Theory of speech perception first introduced by Liberman, Cooper, Shankweiler, and Studdert-Kennedy in 1967, and it is upon this theory that this dissertation will focus. The Motor Theory has endured despite much controversy and some revision. The basic premise of the motor theory of speech perception is that speech is perceived in terms of speech production. Listeners interpret the auditory patterns in terms of the articulatory patterns that would produce auditory patterns like those received. This implies that speech perception and speech production share the same set of invariant articulatory patterns. The premise of the Motor Theory is not necessarily incompatible with the other theories of speech perception discussed previously.

The Motor Theory especially deals with the issue of invariance by maintaining that, although the peripheral articulatory movements and the resulting acoustic speech signal vary, the remote underlying gestures that control the movements are invariant, and that is what allows for recovery of the intended gestures, words, message, etc. of the speaker by the listener. Liberman, et al. maintain that since they share the same set of invariants, speech perception and speech production must be intimately linked through an innate specialized mode that is different from the auditory mode. This speech mode is responsible not only for the perception of the gestures but also for the production of phonetic structures, and is part of the larger specialization for language.

The authors of the original Motor Theory were influenced by work published by Fodor (1983) in which he described the concept of modules. A 
module is a cognitive system that is "domain specific", that is, specialized within the confines of a specific area, using computations that are special to its domain; it is computationally autonomous (so that different systems do not compete for resources, which allows for rapid processing) and is associated with a distinct neural substrate. This substrate does not necessarily have to be one specific location in the brain; it could also be a certain fixed neural network within the brain that consistently acts together to accomplish a specific purpose that makes up a module. Also, modules tend to be "informationally encapsulated", which means that not all of the information being inputted may be actually available to a module, thus keeping the module from being biased by other inputted information. Fodor provides the example of not being able to keep from blinking when a friend, who you know would not hurt you, jabs his finger near your eye. This suggests that the blink reflex has no access to what you know about a person's character.

Largely affected by the concept of "modules," given their previous claims of a "speech mode" of processing, Liberman and Mattingly revised the Motor Theory in 1985. In the revised theory it is claimed that humans possess a biologically inherent neurological module that is specialized to process speech - that is, phonetic elements such as consonants and vowels - which is separate from a more generalized auditory module that processes auditory stimuli. This separate phonetic module is capable of analyzing the variable speech input and it uses this information to discern the speaker's intended gestures. Evidence for specialized neural architecture for linguistic perception goes back to Broca and Wernicke in the 1860s and 
1870s. More recently, Kimura $(1964,1967)$ demonstrated that speech sounds were processed primarily in the left hemisphere, while nonspeech sounds were either processed primarily in the right hemisphere or were not lateralized at all. Ojemann (1983) performed electrical stimulation of the brain and found indications of common cortical areas in the left hemisphere for motor and language functions.

Given the evidence for specialized neural architecture for linguistic processing and the concept of modules as described by Fodor (1983), the revised Motor Theory makes two main claims:

- The objects of speech perception are the intended phonetic gestures of the speaker. Gestures are represented in the brain as remote structures that direct the articulators to move in patterns that are linguistically significant.

- Speech perception and speech production are intimately linked through the specialized phonetic module, such that the module is responsible for both the perception and production of phonetic structures.

This hypothetical perceptual process is automatic and not open to introspection. Listeners perceive phonetic structures directly via rules of speech production, not through several stages of analysis before the combination of all the components of a phoneme occurs, thereby allowing for recognition of a specific phoneme.

Browman and Goldstein (1985) have further investigated the articulatory gesture. They contend that underlying articulatory gestures act as phonological primitives, as well as for analyzing the activity of the vocal 
tract articulators. They consider gestures to be coordinated articulatory movements that are abstract, discrete, and dynamic. In addition, they are invariant across different contexts. Yet, because the gestures are also spatiotemporal, it is possible for them to overlap in time, which results in coarticulation. This system is highly constrained by both the physical system (e. g., the vocal tract) and the dynamics of the articulatory task (e. g., mass, oscillations, position, etc.). Motor Theory views that it is likely that gestures are used as units of perception.

Other theorists consider motor or active theories to be unsubstantiated by the evidence provided, and do not adhere to the idea of separate auditory and phonetic processing modules. Even at an early point in the development of the Motor Theory, Fant (1967) argued that the evidence provided in support of active theories would just as well support passive, sensory-based theories where the decoding process occurs without involving the activation of speech motor centers. He offers an auditory model in which the motor and sensory functions become increasingly involved as the stimulus is processed from the peripheral to the central stages of analysis. Fant assumes that the final destination is the concept of a "message" which is made up of brain centers common to both perception and production. There are separate sensory (auditory) and motor (articulatory) branches, although there is opportunity for communication between the two branches. Auditory input is first processed by the ear and a primary auditory analysis. These incoming auditory signals are then submitted to some kind of direct encoding into distinctive auditory features. Auditory features are then combined in some way to form phonemes, syllables, morphemes, and 
words. This model is very vague and does not account for many of the issues discussed previously, including invariance and segmentation.

Pisoni and Sawusch (1975) advocate an information processing model of speech perception in which speech perception is organized into a hierarchical sequence of events involving structures for storage and processes for the transformation of information over time. During these stages information is transformed, reduced, elaborated, stored, recovered, and used to make various types of decisions. Their stages differ from the stages discussed above in that some of the stages can be in operation simultaneously. Also their model contains mechanisms for short- and longterm storage. When an auditory stimulus is presented it first undergoes a preliminary auditory analysis, then it is processed within a "recognition device" which contains storage areas for sensory information, short-term memory, and long-term memory. Within this device the input stimulus goes through an acoustic feature analysis and a phonetic feature analysis. The features are stored in a buffer, and are then combined to form a phoneme and released as the final output. Phonological, syntactic, and semantic analyses are performed in parallcl and the information can be utilized by lower levels of analysis.

Fowler (1986, 1989) and Fowler and Rosenblum (1992) support a direct-realist theory of speech perception. This theory agrees with the Motor Theory in that Fowler considers the objects of speech perception to be vocal tract activities (gestures). However, in her view, phonetic segments are physical activities of the vocal tract that have linguistic significance and she contends that these vocal tract activities are directly 
perceived by the listener, not through first accessing the motor speech system. Fowler discusses two concepts which help to clarify how phonetic segments are physical activities of the vocal tract: (1) coordinative structure; and (2) phonetic gesture. A coordinative structure is a temporary organization of the articulators that ensures the achievement of the production of an intended phoneme. A phonetic gesture is similar to that described by Browman and Goldstein (1986), that is, phonetic segments of language are composed of phonetic gestures of the vocal tract.

One of the recurring differences in the theories of speech perception presented above is whether speech perception is modular. If one suggests such a strict separation of perceptual functions (auditory vs. specialized phonetic processing), then it is logical to assume the existence of some type of biological neurological substrate for such a division. This is consistent with Fodor's (1983) idea of a module. This dissertation is focused on determining if evidence, in the form of neural activity, of such a neurological separation can be discovered. The idea of a separate neurologically based module for specific function is not so far-fetched, because there are examples in the animal kingdom of such specialized modules. For example, it is thought that biologically coherent systems underlie echolocation in bats (Suga, 1984) and song in birds (Marler, 1970; Thorpe, 1958). There is even evidence in humans that such modularity exists in terms of visual depth perception (Julesz, 1960, 1971; Poggio, 1984).

In order to understand what kind of stimuli might need to be processed within a phonetic module (which would hopefully be observable in the neural activity), it is necessary to describe the linguistic cues that are 
currently thought to compose a phonetic segment. There are a number of acoustic cues which might underlie phonetic distinctions, that is, distinctions among spoken elements such as $/ b /$ and $/ d /$. These acoustic cues that are important for speech perception may also be cues for nonspeech stimuli. For example, it is known that formant transitions are important cues for consonant perception (Liberman, Delattre, Cooper, \& Gerstman, 1954). As was mentioned earlier, a formant is a concentration of energy around certain frequencies, depending on the shape of the vocal tract. A formant transition is a change in frequency over time that occurs as the articulators move from the one articulatory shape to another. Transitions occur rapidly; for example, a formant transition from a stop consonant to a vowel may last for 30 to $70 \mathrm{msec}$.

Mattingly, Liberman, Syrdal, and Halwes (1971) demonstrated that formant transitions were heard differently, depending on whether they were heard within an acoustic speech signal or in isolation. Transitions of the second formant of a two-formant synthesized syllable are sufficient cues for the place distinctions among stop consonants. In isolation, that is, without the following vowel, these formant transitions sound like tone glides or birdlike chirps, that is, completely nonspeech-like. However, when they are embedded in synthetic syllables, unique linguistic events are heard: [bae], [dae], and [gae], with no chirp-like sounds heard at all. Mattingly, et al. found that the synthetic syllables [bae], [dae], and [gae], which contained the second formant transition, showed discrimination functions that were similar to those in response to natural speech, with high peaks at phonetic boundaries and deep troughs within phonetic classes. The formant transition 
heard in isolation, however, showed a discrimination function that was just above chance level. Mattingly, et al. concluded that when an acoustic signal, such as a formant transition, is processed in the phonetic mode, it cues a "nonchirpy" consonant. But when it is processed in the auditory mode, it sounds like a chirp. In other words, when speech is the intended signal, the transitions provide linguistic information, but at other times, as when listening to birdsong, the transitions provide nonspeech information.

If the same acoustic signal can provide information to two different modules, a question arises regarding how this information is shared by the two modules. Liberman and Mattingly (1985) contend that the phonetic module and the auditory module compete for acoustic cues. Whalen and Liberman (1987) further suggest that processing for the phonetic module has priority over processing for general auditory perception.

Although the studies presented above that demonstrate stimuli that evoke two different stimuli or "duplex perception" seem to provide evidence for the existence of a separate speech mode of processing, it could be argued that the stimuli are not being presented in a comparable manner, which could account for the apparent differences in processing. In the above comparisons of speech vs. nonspeech modes of processing, the acoustic context was not held constant; the formant transition was presented in isolation in one case, but as part of a larger acoustic pattem in another. However, it has been demonstrated that the same results are achieved when the acoustic context is held constant, as in the case of a phenomenon known as duplex perception. Duplex perception was first reported by Rand (1974). To evoke a duplex percept, all of an acoustic syllable (e. g., [da] vs. [ga]) 
except only the formant transition that decides between two different consonants (the second or third formant transition), is presented to one ear, and simultaneously the isolated second or third formant transition for either the $/ \mathrm{d} /$ or $/ \mathrm{g} /$ is presented to the other ear. Listeners hear two simultaneous percepts: a [da] or [ga], depending on which formant transition was presented, and a chirp.

To elaborate, consider the following example: There are three formants (representing the resonance frequencies of a human vocal tract) in the acoustic patterns necessary to synthesize a consonant-vowel (CV) syllable. First there is a formant transition (the articulatory movement from the consonant to the vowel) followed by a steady-state frequency (the vowel). As was mentioned previously, formant transitions are important to consonant perception because they help to identify which consonant is being produced. Although differences between two naturally produced $\mathrm{CV}$ syllables typically depend on many acoustic variables, suitable synthetic speech tokens can be created in which all other aspects of the syllable are held constant ${ }^{1}$, with only the second or third formant (F2 or F3) transition providing the distinguishing information between two consonants. In the case of the syllables [da] and [ga], an ambiguous base, consisting of the complete first and second formants and the steady-state portion only of the third formant, tends to be heard mostly as [da], sometimes as [ga]. The addition of a rising F3 transition will be heard unambiguously as [ga], while the addition of a falling F3 transition will be heard unambiguously as [da].

\footnotetext{
${ }^{1}$ Normally, information about the place of articulation in natural tokens are provided by formant transitions as well as burst cues, but in this study, the formant transitions are the acoustic cues being manipulated to create the intended duplex perception.
} 
Both of these F3 transitions, heard in isolation, sound like chirps or glissandi, without any linguistic relevance. However, when these transitions are combined with the syllable base, no chirp is heard (Mattingly, Liberman, Syrdal, \& Halwes, 1971) (Figure 4).

The idea that the same acoustic event can evoke two different percepts, depending on which module processes it, provides a mechanism for examining the claim of a separate phonetic module. Among studies which support the Motor Theory, Mann and Liberman (1983) used this duplex perception paradigm to investigate discrimination functions for this type of stimulus. They systematically varied the onset frequency of the third formant transition across the continuum from $/ \mathrm{d} / \mathrm{to} / \mathrm{g} /$ in eight equal steps. They used the AXB method of discrimination, where A and B are the two stimuli to be discriminated; $X$ is one or the other. The subject's task is to decide if $\mathrm{X}$ is more like $\mathrm{A}$ or more like B. Subjects listened to sets of randomized sequences of six possible combinations of stimuli separated by three steps along the continuum (i. e., by stimulus number; 1 vs. 4,2 vs. 5,3 vs. 6, etc.), with the third formant transition being presented in one ear and the ambiguous base being presented in the other ear. Using the same stimulus tapes, the task was to discriminate either speech sounds amidst detractor nonspeech sounds, or nonspeech sounds amidst detractor speech sounds. In other words, the stimuli were the same, unbeknownst to the subjects, and subjects were given different contexts in which to perform the discrimination tasks. Mann and Liberman found that subjects yielded discrimination functions for these simultaneous duplex stimuli that were very 


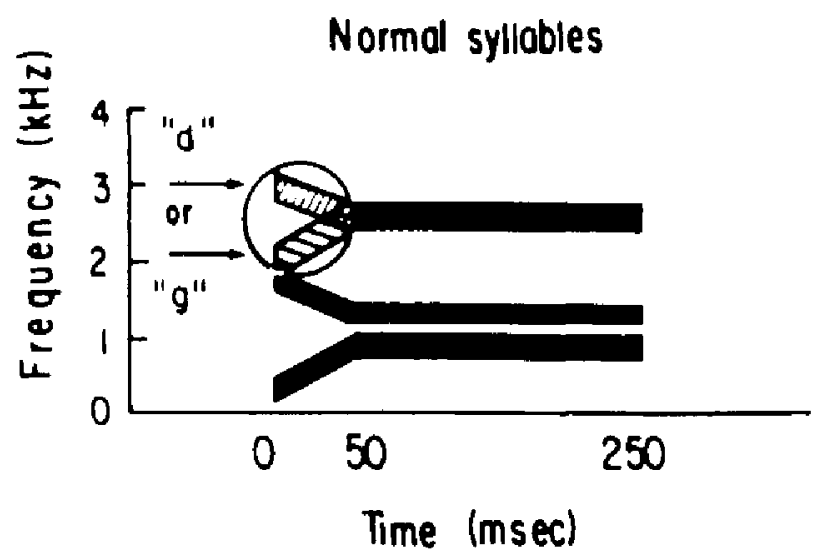

Duplex-producing syllables
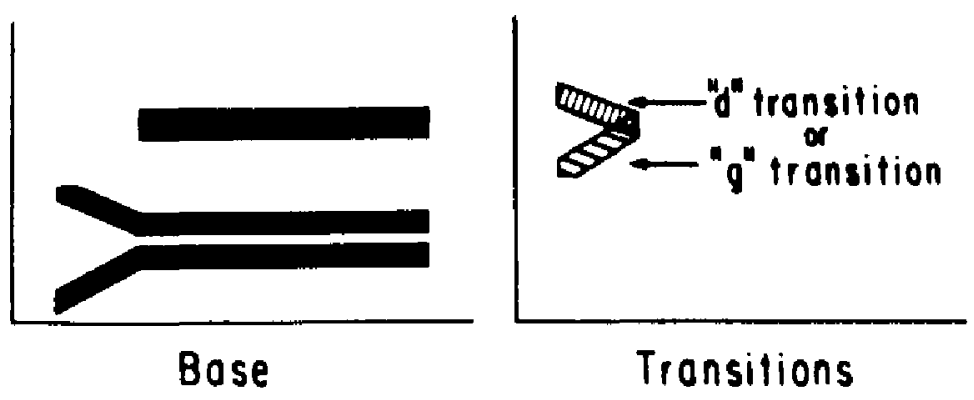

FIGURE 4. Schematic representation of the syllables [da] and [ga], (Whalen and Liberman, 1987). 
similar to those produced separately for (1) the isolated transition, and (2) the whole syllable found by Mattingly, et al. (1971), depending on the context in which subjects were asked to make discriminations, that is, speech or nonspeech.

When subjects identify the syllables during the simultaneous dichotic presentation of the acoustic segments, they are not making a conscious decision to combine the two sets of input to create the single perception of one of the consonants $(/ \mathrm{d} /$ or $/ \mathrm{g} /)$. Mann and Liberman thus claim that because the [da] or [ga] is heard entirely in one ear even though the critical transition cue is presented to the other ear, integration of the acoustic information must be occurring at an automatic perceptual level, rather than at a later, more conscious level of analysis. Both Mann and Liberman (1983) and Whalen and Liberman (1987) thus concluded that these discrimination differences reflect the separate properties of auditory and phonetic perceptual modules.

Questions arise regarding the use of dichotic presentation of the stimuli and the resulting perceptions, such as exactly where and when in the auditory pathway the stimuli are integrated, and what other neural interactions might be affecting the perceptions due to the nature of the stimuli (i. e., in terms of the acoustic environment in which they are presented). Whalen and Liberman (1987) created a form of duplex perception in which the acoustic input was held constant, that is, both percepts (i. e., speech and nonspeech segments) occurred within the same acoustic pattern, as did Mann and Liberman (1983). However, Whalen and Liberman were able to evoke duplex perception by increasing the intensity 
of the third formant transition only in relation to the base. In other words, the third formant transition and the base were not separated and presented to different ears, as in Mann and Liberman's study (1983). Rather the tokens were whole and were presented to both ears simultaneously. Thus, in some of the tokens all of the components were presented at the same intensities and subjects perceived only the CV syllable. In other tokens, the intensity of the F3 transition was increased relative to the base and subjects perceived both the C.V syllable and a tone glide. At relatively low intensities, the transitions serve only to cue the intended consonant. But at higher intensities, the $\mathrm{F} 3$ transition performs the dual task of providing a linguistic cue in the perception of the consonant and a nonlinguistic cue in the perception of a nonspeech chirp.

Whalen and Liberman also used sinusoids to represent the F3 transitions. This was done to reduce the likelihood that any linguistic information could be obtained from the F3 transitions in isolation. Sinusoids are simple pure-tone sine waves or tone glides, unlike formants in natural speech which are complex combinations of different harmonics. Duplexity thresholds (the lowest intensity level of the F3 transition at which both a syllable and tone glide were detected) were determined for both syllables. Both parts of the signal were presented to both ears, and when a token was presented that had the third formant transition intensity increased, listeners simultaneously heard a tone glide and a whole unambiguous syllable.

Presenting all parts equally to both ears avoids some of the problems discussed previously that may be involved with dichotic presentation. Whalen and Liberman maintain that since this duplex perception occurs 
when the acoustic input is held constant, it cannot be attributed to any auditory interactions arising from changes in acoustic context or to a shifting of attention between two forms of an ambiguous signal. They concluded that their results also support the existence of separate auditory and phonetic modes of perception. In addition, they claim that their results indicate that phonetic module processes have priority over the auditory module. Since a tone glide is not perceived until the intensity of the third formant transition is increased relative to the base, they surmise that the incoming signal is processed first in the phonetic module for its linguistic information and that is why a syllable is heard without a tone glide. The remainder is then forwarded to the auditory module for processing, at which point a tone glide is heard.

Fowler and Rosenblum (1992) interpret Liberman and Mattingly's (1985) and Whalen and Liberman's (1987) results differently. They argue that Whalen and Liberman's interpretation, namely that the phonetic mode takes precedence over the auditory mode, provides a very different perspective from earlier duplex perception studies. Earlier reports of duplex perception maintained that the same acoustic signal was being perceived in two ways simultaneously, that is, the third formant transition was being perceived as a nonspeech chirp concurrently with being a specific $\mathrm{CV}$ syllable. What Whalen and Liberman are now maintaining is that part of the formant transition integrates with the syllable and the remainder is heard as a chirp. Fowler and Rosenblum (in press) conducted a study in which they looked for the phenomenon of duplex perception using nonspeech sounds. They predicted that duplex perception would occur whenever two conditions 
were met: (1) a pair of acoustic fragments is presented that, when integrated, represent a natural occurring event; and (2) one of the fragments is unnaturally intense. Under these conditions, the integrated event should take precedence over the isolated fragment and the intense fragment should be duplexed regardless of the type of natural sound-producing event that is involved (speech vs. nonspeech). They chose an event which occurs naturally in the environment, but one which could not, biologically and developmentally, be argued to have a module: a slamming metal door. They digitized a recording of a metal door slamming and then fragmented the signal such that there was a base that sounded like a wooden door being slammed, and an isolated segment (the equivalent of the isolated third formant transition in the speech studies) which sounded "like a can of rice being shaken." When combined, of course, the signal sounded like a metal door slamming. There were three ranges of intensity: (1) the intensity was well below the natural intensity relationship of the chirp to the base; (2) the intensity was within the range of the natural intensity of the relation; and (3) the intensity was well above it. They presented the stimuli to subjects in a random order and subjects were to write what they heard on answer sheets.

The results showed that at the low intensity levels, subjects heard the base only (a wooden door slamming). At normal intensity levels, subjects mostly heard a metal door slamming. At increased intensity levels, subjects heard both the metal door slamming and a separate shaking sound. Because subjects heard two different percepts given one signal (albeit, at an increased intensity level) Fowler and Rosenblum consider the nonspeech percept to be duplex. Because the separate shaking sound was heard only at 
the increased intensity level, they consider the natural event of the metal door slamming to take precedence over the chirp portion. Fowler and Rosenblum suggest that processing precedence occurs when a chirp fills a "hole" in a simultaneously presented acoustic signal so that together the two parts of the signal specify some sound-producing natural event in the environment. If anything is left over after the hole is filled, the remainder is heard as separate. They maintain that this evidence argues against the hypothesis that there is a speech module that is biologically significant since the same duplex perception phenomenon and processing precedence occurs with nonspeech stimuli.

Again, if a phonetic module exists that is separate from an auditory module, then it should be instantiated in different patterns of neural activity. It would be helpful in gaining more insight into this issue if there were neurological evidence which demonstrated the distinction between an auditory module and a phonetic module. One method of monitoring online, in vivo neural functions utilizes electroencephalograms (EEGs). EEGs are recorded from the scalp and reflect the fluctuating voltages in electrical activity from neurons in response to both environmental stimuli and stimuli within an individual. When there is a change in the electrical activity of neurons that is temporally associated with physical stimuli or psychological processes, it is known as an event-related potential (ERP). ERPs can be classified as exogenous or endogenous. Exogenous potentials are largely determined by the physical parameters of a stimulus, such as the modality in which the stimulus is presented, while endogenous potentials can occur independently of external stimuli and are mostly affected by psychological 
parameters, for example, improbable stimuli. ERPs have a number of components; early components are usually exogenous and later components are usually more endogenous. ERPs are usually too small to be distinguishable from the background EEG. Electrical recordings collected at the same point in time after a repeated stimulus event are averaged together. Signal averaging of multiple trials allows ERPs to become more readily apparent. Background EEG not related to the stimulus is mostly random and tends to cancel itself during the averaging process. Thus, ERPs, being temporally consistent for every event, will remain constant during each trial. The ERPs will therefore become more salient compared to the background EEG during the averaging process (Figure 5) (Picton \& Stuss, 1984).

ERPs are an effective technique for monitoring cognitive processes involved in speech and language processing because they are a reflection of the underlying neural events that occur as a result of cognitive processing. Cognitive processes occur rapidly (in milliseconds) and ERPs have the temporal resolution to track this rapid processing. A number of components have been identified in an ERP which are a reflection of various psychophysiological and psychological processes. For example, there is a component known as the $\mathrm{P} 300$ that has been associated with task relevance and expectancy (Picton and Stuss, 1980). The notation commonly used to describe components should be clarified: the letter (e. g., "N" or "P") refers to polarity, either negative or positive, and the number (e. g., "300") refers to the number of milliseconds until a peak is reached following the taskrelated stimulus. This number is not exact in that it reflects an average of a range of time in which a response occurs. (Another way that is used to 


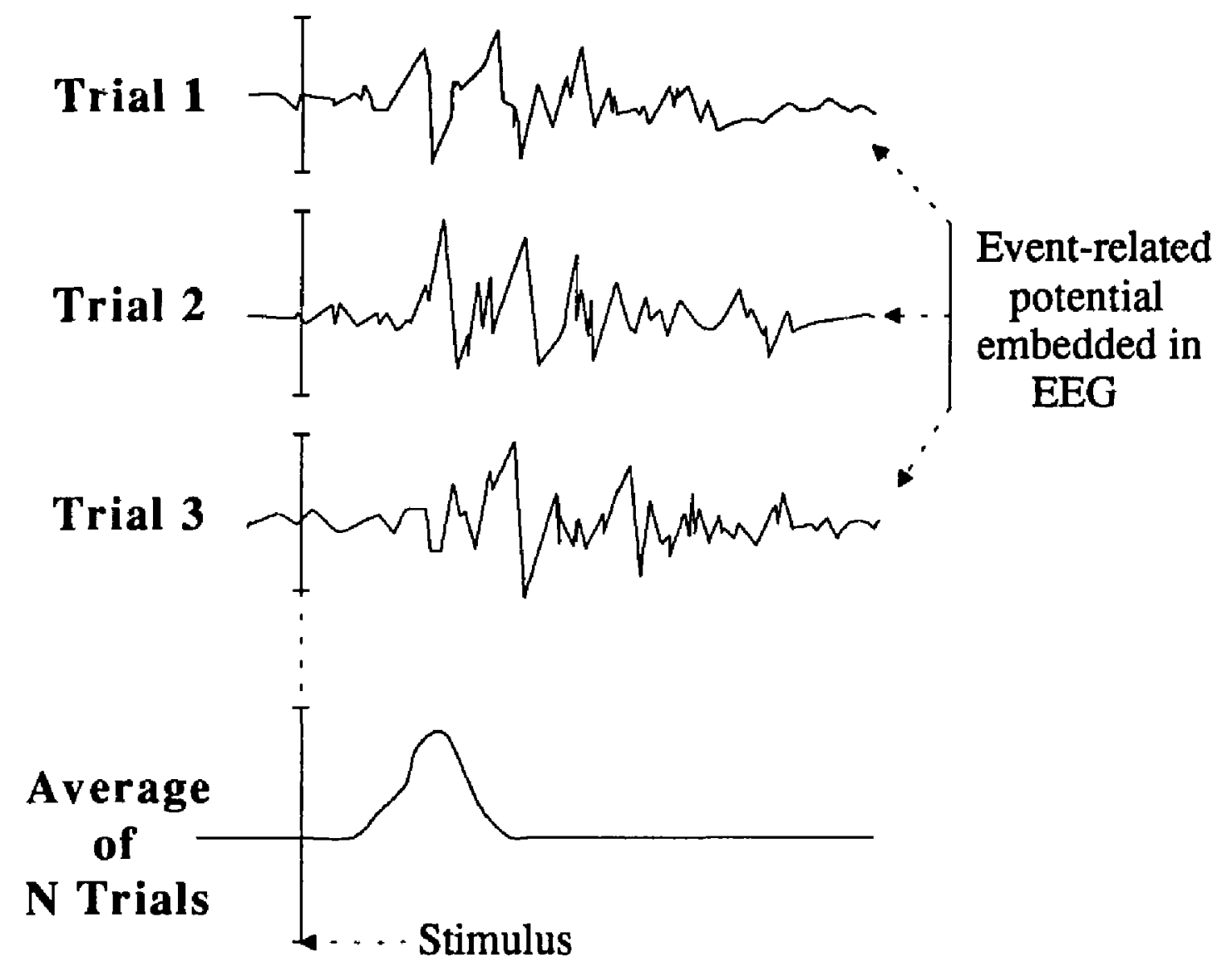

FIGURE 5. Schematic of ERP averaging process. Multiple trials are averaged together and background EEG not related to the stimulus cancels itself out, resulting in a waveform with more distinctive peaks. 
describe the components is to refer to the peaks in the order in which they occur. For example, the first negative peak is labeled "N1", the first positive peak as "P1", the second negative peak as "N2", etc. This is the terminology that will be used in this study). Thus the P300 is a positive peak that occurs about $300 \mathrm{msec}$ after stimulus onset. Typically the P300 is evoked utilizing what is known as an "oddball" paradigm. In this paradigm one stimulus (say a $1000 \mathrm{~Hz}$ tone) is presented $80 \%$ of the time and another stimulus (a $2000 \mathrm{~Hz}$ tone) is presented $20 \%$ of the time. When subjects' ERPs are recorded, their waveforms contain a positive peak that occurs approximately $300 \mathrm{msec}$ following the presentation of the less frequent (oddball) tone. This peak does not occur following the presentation of the frequent tone. Since subjects for the most part hear the frequent tone, the occurrence of the infrequent tone is unexpected, so the P300 is thought to represent neural activity involved in processing unexpected stimuli (Picton and Stuss, 1980).

The N400 has been associated with various semantic relationships among words in a sentence or a list of words (Picton \& Stuss, 1980). Much work has been done using ERPs in several areas of speech and language processing, especially in the area of semantic language processing (Kutas and Hillyard, 1980-1989; Boddy, et al., 1981; Fischler, et al., 1982; Stuss, et al., 1983; Molfese, 1983, 1984, 1988; Garnsey, et al., 1989). The work has mostly utilized visual evoked potentials (VEPs). Kutas and Hillyard (1980) recorded an N400 following a semantically incongruous word occurring at the end of a sentence (e. g. "I take coffee with cream and dog."). Molfese and Schmidt (1983) used auditory stimuli to investigate consonant 
perception in different vowel environments and upon performing a Principal Components Analysis, found a number of components that differentiated between various aspects of the stimuli.

No studies have been done in which ERPs were used to investigate the existence of separate auditory and phonetic modules. Some studies have used ERPs to examine processing of phonetic segments. Wall, Fox, Moenter, and Dalebout (1991) examined the latency and amplitude of the P300 in young adult (19-25 years) and older subjects (61-75 years) while they listened to tones, CV syllables, and isolated vowels. Latencies of the elderly subjects were longer for the CV syllables and vowels than for the tones. The latency differences among the vowel stimuli seemed to reflect not only a category distinction based on phonemic differences between two vowels, but acoustic differences as well. Wall, et al. suggest that these data reflect the operation of an acoustic level of perceptual processing and a phonetic level of perceptual processing. They also found that P300 amplitudes were greater for the tone stimuli than for the speech stimuli. These findings lend support to the idea that ERPs may provide evidence as to the difference in auditory and phonetic processing.

Some early studies attempted to differentiate the ERPs to speech vs. nonspeech stimuli, mostly in terms of whether one cerebral hemisphere processed one type of stimulus more than another type of stimulus (Tobin, 1968; Greenberg, 1970; Roth, et al., 1970;), but there were conflicting results. Cohn (1971) recorded ERPs to clicks and consonant-vowelconsonant (CVC) words from 4 electrodes placed over the left and right side of the head, one pair located $2 \mathrm{~cm}$ anterior to the external meatus and a 
second pair located $2 \mathrm{~cm}$ from the midsagital line in the vertical coronal plane. For the word stimuli, 17 adult subjects produced larger left hemisphere (LH) ERPs. A large positive component was reported over the right hemisphere $(\mathrm{RH})$ in all 37 subjects in response to the clicks. Ratliff and Greenberg (1972) compared the ERPs of spoken words and comparable nonlinguistic noise. They found differences in latency and polarity of components for each type of stimulus. They identified the components in order of their occurrence P0, N1, P1, N2, etc., according to polarity. For the linguistic stimuli they identified a N1, P1, N2, P2, N3, P3, N4, and P4. For the nonlinguistic stimuli they identified a P0, N1, P1, N2, P2, N3, P3, and N4. The ERPs to nonlinguistic stimuli contained an early positive component ( $\mathrm{P} 0$, with a mean latency of $23.1 \mathrm{msec}$ ) and a late negative component ( $\mathrm{N} 4$, with a mean latency of $456.2 \mathrm{msec}$ ) not observed in the linguistic stimuli. Other component latencies in the ERPs to nonlinguistic stimuli were generally later than those for linguistic signals. ERPs to linguistic stimuli were more similar to each other than to the ERPs from their matched nonlinguistic equivalents. There was no description of scalp topographical electrical activity distribution.

The observation that most of the components of the linguistic stimuli tended to have shorter latencies led Ratliff and Greenberg to conjecture that there is a "language-prionity system" for evaluation of auditory stimuli, in which linguistic stimuli are identified and processed rapidly at higher centers. Nonlinguistic stimuli would take longer to process because the system is trying to assign some meaning to the signals. Ratliff and Greenberg consider that there may be a speech processing mode and a 
nonspeech processing mode. A shorter latency for linguistic stimuli is the opposite of the results obtained by Wall, et al. (1991), in which linguistic stimuli had longer latencies than nonlinguistic stimuli. In their study, however, Ratliff and Greenberg (1972) did not control for noncerebral activity such as eye movement, which could have confounded the results.

The results from Wall, et al. (1991) and Ratliff and Greenberg (1972) that the linguistic and nonlinguistic stimuli appeared to be processed differently in terms of latency lends some support to the Motor Theory's claim regarding two different modules for processing auditory signals, one for speech and another for nonspeech signals.

Other investigators have included a behavioral task while recording ERPs so that the subject's attention and involvement during testing can be controlled. Neville (1974) found that the P1-N1 amplitude of the response to dichotic verbal stimuli was larger over the left hemisphere than over the right, whereas there was no significant asymmetries for dichotic clicks. The dichotic verbal stimuli elicited shorter latencies in the $\mathrm{N} 1, \mathrm{P} 1$, and $\mathrm{N} 2$ components of the ERPs from the left hemisphere. The verbal stimuli were recordings of the monosyllabic digits from one to ten, each having a duration of $100 \mathrm{msec}$. The clicks each had a duration of $10 \mathrm{msec}$. Neville contends that these results lend support for the hypothesis of asymmetry of hemispheric functioning; however, there currently is much controversy regarding left and right ear advantage studies, and other studies have not been able to replicate Neville's results (Galambos, et al., 1975).

Hillyard and Woods (1979) increased task demands and recorded ERPs during the processing of high information content stimuli and tones. 
Twelve subjects listened to monotone speech stimuli as part of a coherent, ihymed poem. Subjects were instructed that they would be required to answer difficult questions about the content later. Tones of varying frequencies $(400 \mathrm{~Hz}, 1000 \mathrm{~Hz}$, and $4000 \mathrm{~Hz})$ were presented at the same rate as the words and were matched with the words in range of loudness, interstimulus interval, and rise/decay times. Electrodes were placed over the vertex, at a left scalp region over Wernicke's area, and its RH homologue. The speech stimuli elicited a $34 \%$ larger N1 component, and a larger (18\%) negative potential from 100 to $500 \mathrm{~ms}$ relative to the tone stimuli. The $\mathrm{N} 1$ response from the speech stimuli was largest over the LH, but no such differences were noted for the tone stimuli.

In regard to the innateness of speech perception, Novak, et al. (1989) obtained auditory ERPs to synthesized syllables ([da] and [ta]) and to the isolated formants $\mathrm{F} 1, \mathrm{~F} 2$, and $\mathrm{F} 3$ of [da] at monthly intervals from children from birth to three months and at six months. They used a bilateral array of 16 active electrodes, which allowed for topographic analysis. They found a maturational sequence in terms of ERP waveforms which coincided with maturation of the auditory cortex, but found no difference in maturation of ERPs in terms of the different center frequencies (i. e., the steady-state portion of the formant that occurs after the transition) of the formant stimuli. Since auditory ERPs may be manifestations, in part, of cortical synaptic activity elicited by auditory stimuli, the pattem of anatomical maturation of the auditory cortex should be related to the developmental sequence of the cortical auditory ERP. The differential maturational pattern in response to the full syllables over midline and lateral scalp suggests temporally 
overlapping contributions from at least two bilateral cortical regions, which they consider to be located in the superior temporal plane and in the lateral surface of the superior temporal gyrus, respectively. A systematic topographical difference in the ERPs of the individual formants (F1, F2, F3) of [da] was not observed. They observed certain consistent patterns of hemispheric asymmetry at three and six months for the full syllables [da] and [ta], and not so much for the individual formants, which were not present during the first two months of life. However, there was considerable individual variation among subjects, so group differences were not highly significant. No observations were made regarding any other differences between the speech and nonspeech stimuli. They also did not find any topographic difference in the ERPs elicited by each of the three formants, which had different center frequencies.

Molfese, Freeman, and Palermo (1975) investigated changes in ERP responses over different developmental periods to speech and nonspeech stimuli. They recorded ERPs from T3 and T4 of ten infants one week to ten months, eleven children four to eleven years old, and ten adults 23 to 29 years old. The natural speech stimuli consisted of CV syllables and monosyllable words. A piano chord and white noise burst served as the nonspeech stimuii. In general, larger LH amplitudes were found for the speech stimuli in all groups of subjects while larger RH amplitudes were noted for the nonspeech material. Molfese and his colleagues concluded that such results indicate that from infancy onward ERP can distinguish speech from nonspeech stimuli. 
In another study using speech and nonspeech stimuli, Molfese \& Molfese (1988) investigated auditory ERPs from preschool children to temporal cues to speech ([ka] and [ga]) and nonspeech stimuli (two-formant tones) (Voice Onset Time and Tone Onset Time). The speech stimuli contained three formants and had variable VOT values of $0-,+20-,+40-$, and +60 -msec, $(0-$ and +20 -msec VOTs were identified by subjects as [ga]; +40 - and +60 -msec VOTs as [ka]). The nonspeech stimuli had the lower frequency sinusoidal tone delayed by the same values as the VOTs above, relative to the higher frequency sinusoidal tone. Molfese and Molfese performed a Principal Components Analysis (PCA), an analysis procedure that yields a set of components contributing independently to the experimental variance of the ERP waveform. They found that temporal delays for both speech and nonspeech auditory materials appear to be processed in the right hemisphere temporal lobe. The PCA revealed components in the right hemisphere around $180 \mathrm{msec}$ and $400 \mathrm{msec}$ poststimulus onset that discriminated the 0 - and $20-\mathrm{msec}$ stimuli ([ga]) from the 40- and 60-msec stimuli ([ka]). Although this study used speech and nonspeech stimuli, the object of interest was the nonphonetic element of temporal delay. There is no discussion of differences in processing of the actual speech and nonspeech stimuli.

Molfese and Schmidt (1983) evaluated ERPs to consonants within different vowel environments. The twelve different stimuli consisted of two sets of CV syllables: [bi], [bae], [b ], [gi], [gae], and [g ]. The six syllables of the first set contained normal speech-like formant structure. The second set contained stimuli with sinewave formant structure. No nonspeech 
stimuli were included. Upon performing Principle Components Analysis, a number of components were identified that apparently reflected various aspects of the stimulus parameters. They found that one component around $170 \mathrm{msec}$ post-stimulus reflected changes over both hemispheres in response to consonant changes only. Another component reflected changes over only the left hemisphere to different consonants regardless of their vowel environment. A third component reflected change for the different consonants depending on their vowel environment. A number of components were identified that were sensitive to the formant structure variable. For example, one component occurring at $245 \mathrm{msec}$. seemed to reflect activity over both hemispheres which discriminated between normal and sinewave formant structures, while another later-occurring component reflected left hemisphere activity only that distinguished between the normal and the sinewave formant stimuli. Molfese and Schmidt suggest that this indicates that consonant perception initially involves mechanisms over both hemispheres which are sensitive to acoustic information, while other, more lateralized, mechanisms involve the further processing of linguistic information.

The results obtained in the above studies are inconsistent from study to study. These inconsistencies may be explained by several factors. First of all, most of these studies did not control adequately for noncerebral artifact such as eye movement, and such artifact could have contaminated the data. Secondly, because there are so many features which characterize the difference between speech and nonspeech stimuli, it is impossible to specify exactly what factors produce these effects. Another problem with 
most (if not all) of these early studies is that the speech and nonspeech stimuli were not matched in terms of such acoustical characteristics as frequency, formant structure and duration, number of formants, frequency transitions, etc. This makes it more difficult to ascertain differences on the basis of linguistic characteristics of the speech stimuli vs. the nonspeech stimuli, or to the multiple acoustic differences which separate the two classes of stimuli.

As a whole, the ERP studies outlined above demonstrate the utility of using ERPs as probes of speech processing in the brain. The study by Molfese and Schmidt (1983) could even be said to provide support for the idea of stages of auditory processing, with bilateral processing of acoustic information occurring early, followed by later left-lateralized processing of linguistic information. The duplex perception studies lend support to one of the claims of the motor theory of speech perception: that there exists a specialized module which processes linguistic signals that is separate from an auditory module which processes general auditory signals. However, there has been no cohesive work that has exploited the unique phenomenon of duplex perception while simultaneously observing the resulting associated brain electrophysiological activity. Such a study could contribute more definitive evidence regarding this intriguing question and it is toward this end that this study is directed.

To examine this issue, scalp electrical activity could be monitored during a duplex perception task, and data could then be analyzed for a) differences associated with speech vs. nonspeech stimuli in scalp electrical activity at a single electrode location across time; and b) differences 
associated with speech vs. nonspeech stimuli among electrodes across the scalp at any given time.

This dissertation will attempt to answer the following general question:

Is there neurological evidence supporting the existence of separate cognitive processing modules (as proposed in the literature, Mattingly and StuddertKennedy, 1991): one devoted to the analysis of "nonspecialized" auditory signals, and one devoted to the extraction of linguistic information?

Results from studies previously discussed which investigated the issue of speech/nonspeech processing were weakened because equivalent speech and nonspeech stimuli were not used, and limited because few electrode sites were recorded. To avoid these limitations, the methodology in this study seeks to:

1. Develop a highly controlled set of stimuli that represent equivalent speech and nonspeech stimulus tokens such that the differences are limited to a single acoustic parameter: amplitude.

2. Use these ideal stimuli in an ERP study to determine whether they could be used reliably to elicit ERP differences. 
3. Set the stage for further studies by collecting data from not just one electrode site, but from a full complement of electrodes, which would provide scalp topographical information.

Simply, brain ERPs will be recorded while subjects listen to and silently identify stimulus tokens designed to elicit duplex perception by having a third formant transition with increased amplitude. The independent variable will be the stimulus token. The dependent variables will be the amplitude of the maximum excursion in microvolts and the time of the maximum excursion in milliseconds. Assuming the existence of a specialized phonetic module, as specified by the Motor Theory, and assuming that the operations of this module are neurologically differentiable from those of general auditory processing, then the following hypotheses can be made:

1. There will be a significant difference in the ERP waveforms of speech and nonspeech stimuli in terms of their latencies:

A. CV syllables with equal formant amplitudes (speech only tokens) will have shorter latencies than CV syllables with an increased amplitude of only the F3 transition (speech + nonspeech tokens) (i. e., [da] vs. [da] + tone glide; [ga] vs. [ga] + tone glide). 
B. Comparison of $\mathrm{CV}$ syllables with an increased amplitude of only the F3 transition (speech + nonspeech tokens) should indicate relatively equal latency values related to stimulus type. Any differences found would likely be in response to the different initial consonant since all other factors are held constant. The [da] + tone glide would be compared to the $[\mathrm{ga}]+$ tone glide.

C. Comparison of $\mathrm{CV}$ syllables with equal formant amplitudes (speech only tokens) should indicate relatively equal latency values related to stimulus type. Any differences found would likely be in response to the different initial consonants because all other factors are held constant. The [da] would be compared with the [ga].

D. Comparison of the tone glide stimuli (nonspeech only tokens) should indicate relatively equal latency values related to stimulus type. Any differences found would be in response to the different tone contour since all other factors are held constant. The isolated [da] tone glide would be compared with the isolated [ga] tone glide. 
2. There will be a significant difference in the ERP waveforms of speech and nonspeech stimuli in terms of their base-topeak amplitudes.

In Hypothesis 1A, the speech tokens are compared with the speech + nonspeech tokens. Given this situation, it would be expected that different neural activity would be observed for speech vs. nonspeech tokens. It is expected that speech only tokens would have shorter latencies than the speech + nonspeech tokens because, as Whalen and Liberman (1987) maintain, the latter token types would first have the linguistic portion of the signal processed by the phonetic module, followed by the processing of the remaining nonlinguistic portion by the auditory module. Pure speech tokens, on the other hand, only have a linguistic portion to be processed, so processing should not take as long. Specifically, [da] and [ga] should have shorter latencies than [da] + tone glide and [ga] + tone glide.

In Hypotheses 1B, 1C, and 1D, it would be expected that latency values would generally be relatively equal since the tokens being compared contain the same components, that is, pure speech vs. pure speech, speech + nonspeech vs. speech + nonspeech, and nonspeech only vs. nonspeech only. Any differences would have to be a reflection of the differential processing required for the different initial consonants $(/ \mathrm{d} /$ and $/ \mathrm{g} /)$ in Hypotheses $1 \mathrm{~B}$ and $1 \mathrm{C}$, and for the different tone contours in Hypothesis 1D.

In Hypothesis 2, it is difficult to predict a direction in which such differences might occur given the different interpretations applied to amplitude values. Increased amplitudes could be an indication of more 
resources (cells being activated) being utilized for a given process. On the other hand, if one assumes that amplitude is a reflection of the number of cells being activated for a given process (the more cells that are activated, the higher the amplitude), then decreased amplitudes could be an indication of more ongoing activity occurring in a given cortical area such that any new stimulus arriving at that area would find fewer cells that are not already busy with another activity to activate. With fewer cells being activated for a given activity, ERP amplitudes would not be so high (Grabow, Aronson, Rose, and Greene, 1980). Therefore, it is only hypothesized that there will be some kind of difference between the amplitudes of the speech and nonspeech stimuli, but nothing more specific can be conjectured.

If there are significant differences in the ERP waveforms of the speech and nonspeech stimuli, this will support the contention of the motor theory of speech perception that there is a separate neurological module for processing linguistic signals. If significant differences are not obtained, the ERP evidence could be argued to support those theories which do not so radically separate the processing of speech signals from nonspeech signals. However, a failure to obtain such differences may simply reflect the limitations of the current research design, or the technology used. 


\section{CHAPTER II METHODOLOGY}

In this study responses to a duplex perception task were monitored via brain electrophysiological activity (event-related potentials). Neural activity was searched for that would indicate that the processing associated with general auditory stimuli and linguistic stimuli is significantly different. Such a result would support the claim of the motor theory of speech perception that there is a specialized speech processing module that is separate from a nonspeech processing module.

Subjects

Twenty-one female subjects ages 19-39 years (mean age: 22.5 years) with at least a high school education participated in this study. Undergraduate and graduate students who were majoring in speech and hearing sciences or were enrolled in an introductory speech and hearing disorders course were solicited to become subjects. Recently some evidence has been presented which suggests that brain potentials in women fluctuate during their menstrual cycles, (Johnston \& Wang, 1991), although Fleck and Polich (1988) found no relation between the P300 and the menstrual cycle. To control for this possible fluctuation, subjects were 
tested during their menses. It is during this time frame that they would have more similar hormone profiles (Dr. Elizabeth Kennard, Reproductive Endocrinologist, personal communication, January, 1992). Also, since many studies acknowledge that brain potentials can change according to circadian rhythm (Broughton, et al., 1988), subjects were tested within the same period during the day, between 12:00 and 6:00 p.m. By performing testing at the same relative time for each subject, fluctuations in hormone levels and/or body function timing should occur in all subjects similarly. All subjects had normal hearing in both ears with pure tone air conduction thresholds of $25 \mathrm{dBHL}$ or better at frequencies $500,1000,2000,3000$, and $4000 \mathrm{~Hz}$. Subjects had no history of head injury with an associated loss of consciousness and were taking no medication or substance that is known to affect neurological functioning (e.g., antihistamines, etc.). They were tested for handedness using a handedness inventory (adapted from Oldfield, 1971), and only those subjects who were determined to be right-handed with no immediate family members (i.e., parents and siblings) who were left-handed were chosen to participate. All subjects were native speakers of English. Three subjects' data could not be included in the study because there was too much artifact and no distinctive peaks could be discerned.

\section{Stimuli}

The stimuli were based on those created in Whalen and Liberman's (1987) study. The stimuli represented synthesized versions of the consonant-vowel (CV) tokens [da] and [ga] which were $250 \mathrm{msec}$ in total 
duration. Two sinusoids representing the F3 transitions for [da] and [ga], 50 msec in duration, were created. The CV tokens consist of two parts: 1) a base, which is the same for all tokens, consisting of the first two formants and the steady-state portion of the third formant and 2) a third formant transition in the form of a time-varying sinusoid that follows the center frequencies, that also varies in amplitude (see Figure 4). The base was created using the Klatt software synthesizer (as modified by Y-Y. Qi and K. Johnson in the Linguistics Lab at The Ohio State University, 1988) implemented on an IBM-compatible computer, using the parallel model rather than the cascade model (Klatt, 1980). The sinusoids were created with a different software synthesizer (written in $\mathrm{C}$ by Chien-Yeh Hsu in the Division of Speech and Hearing Sciences at The Ohio State University, 1991) that generates pure tones and tone glides. Parameter values which represent frequencies, bandwidths, and amplitudes of an exemplar [da] and [ga] (Tables 1 and 2) were inputted. All parameter values were based on those suggested by Klatt (1978).

There is enough information in the release burst of a stop consonant to identify the consonant, and the release bursts of $/ \mathrm{d} /$ and $/ \mathrm{g} /$ are very different, so in order to prevent the identification of the tokens based on release burst information, there was no stop release burst. Fundamental frequency (F0) of the base began at $120 \mathrm{~Hz}$ and dropped linearly to $110 \mathrm{~Hz}$ by the end of the token, which produced a more natural sounding speech token. The base and one of the transitions were combined in a timesynchronous manner to produce a single stimulus token. These tokens were outputted directly from the computer to headphones. This direct output was 
TABLE 1. Parameter values of an exemplar [da] (formant frequencies, bandwidths, and amplitudes).

\begin{tabular}{cccccccccccc}
\hline \multicolumn{10}{c}{ Synthesis Parameter Values } \\
\hline $\begin{array}{c}\text { time } \\
\text { (msec) }\end{array}$ & f0 & F1 & b1 & F2 & b2 & F3 & b3 & a1 & a2 & a3 \\
\hline 0 & 1100 & 374 & 70 & 1705 & 95 & 2800 & 180 & 60 & 60 & 60 \\
35 & 1200 & 627 & 70 & 1327 & 79 & 2644 & 167 & 60 & 60 & 60 \\
45 & 1200 & 700 & 70 & 1220 & 74 & 2600 & 163 & 60 & 60 & 60 \\
55 & 1200 & 700 & 70 & 1220 & 70 & 2600 & 160 & 60 & 60 & 60 \\
250 & 1200 & 700 & 70 & 1220 & 70 & 2600 & 160 & 60 & 60 & 60 \\
\hline
\end{tabular}


TABLE 2. Parameter values of an exemplar [ga] (formant frequencies, bandwidths, and amplitudes).

\begin{tabular}{cccccccccccc}
\hline \multicolumn{10}{c}{ Synthesis Parameter Values } \\
$\begin{array}{cccccccccccc}\text { time } \\
\text { (msec) }\end{array}$ & f0 & F1 & b1 & F2 & b2 & F3 & b3 & a1 & a2 & a3 \\
\hline & 1100 & 374 & 70 & 1705 & 95 & 2175 & 180 & 60 & 60 & 60 \\
35 & 1200 & 627 & 70 & 1327 & 79 & 2505 & 167 & 60 & 60 & 60 \\
45 & 1200 & 700 & 70 & 1220 & 74 & 2600 & 163 & 60 & 60 & 60 \\
55 & 1200 & 700 & 70 & 1220 & 70 & 2600 & 160 & 60 & 60 & 60 \\
250 & 1200 & 700 & 70 & 1220 & 70 & 2600 & 160 & 60 & 60 & 60 \\
\hline
\end{tabular}


accomplished by having each synthesized waveform in digital form converted to an analog signal using a 12-bit digital-to-analog converter (DT2801) running at $10 \mathrm{kHz}$. Output was low pass filtered at $4.5 \mathrm{kHz}$ between the D/A converter and the headphones. Subjects heard the tokens binaurally at $75 \mathrm{dBSPL}$, with an interstimulus interval (ISI) of $1646 \mathrm{msec}$. Level of signal was measured using a B \& K type 2209 Sound Level Meter with its associated 4152 artificial ear and 1613 filter, using a B \& K 4144 microphone.

The base, when presented in isolation, was heard as an ambiguous CV syllable, mostly as [da]. When the sinusoids were presented in isolation, they were heard as nonspeech tone glides. When one of the sinusoids was combined with the base at an amplitude equal to the base, the stimulus token was heard unambiguously as the $\mathrm{CV}$ syllable corresponding to whichever sinusoid was included, i.e., either the [da] or [ga] F3 transition. Under these same conditions, with the exception that the amplitude of the sinusoid only was increased, the appropriate CV syllable and a tone glide were both heard, simultaneously. The above perceptions were obtained through informal testing from the committe chair and two pilot subjects. Thus, the following six types of tokens were presented to subjects within various experimental conditions:

1. A base + sinusoidal F3 transition for [da] with equal amplitude of all formants (this will produce the pure speech token [da]).

2. A base + sinusoidal F3 transition for [ga] with equal amplitude of all formants (this will produce the pure speech token [ga]). 
3. A sinusoidal F3 transition for [da] presented at the same increased amplitude as in \#5 and \#6 below (this will produce a pure nonspeech tone glide).

4. A sinusoidal F3 transition for [ga] presented at the same increased amplitude as in \#5 and \#6 below (this will produce a pure nonspeech tone glide).

5. A base + sinusoidal F3 transition for [da] with increased amplitude of F3 transition, relative to the amplitude of the base (this will produce the simultaneous speech token [da] + the nonspeech tone glide).

6. A base + sinusoidal F3 transition for [ga] with increased amplitude of F3 transition, relative to the amplitude of the base (this will produce the simultaneous speech token [ga] + the nonspeech tone glide).

Procedure

Electrophysiologic activity from subjects was monitored while subjects listened and silently identified each token as it occurred. This is unlike the typical oddball paradigm in which subjects make some type of overt decision regarding the stimuli (e. g., by stating the number of infrequent stimuli they heard). There were five conditions:

1. [da] vs. [da] + tone glide: the CV base combined with a [da] F3 sinusoidal transition of equal amplitude was paired with the $\mathrm{CV}$ 
base combined with a [da] F3 sinusoidal transition of increased amplitude. Subjects were asked to silently identify each token as being either a plain [da] or a [da] with a chirp. This condition was intended to provide data about processing a pure speech stimulus vs. a simultaneous speech + nonspeech stimulus.

2. [ga] vs. [ga] + tone glide: the CV base combined with a [ga] F3 sinusoidal transition of equal amplitude was paired with the $\mathrm{CV}$ base combined with a [ga] F3 sinusoidal transition of increased amplitude. Subjects were asked to silently identify each token as being either a plain [ga] or a [ga] with a chirp. This condition was intended to provide data about processing a pure speech stimulus vs. a simultaneous speech + nonspeech stimulus.

3. [da] + tone glide vs. [ga] + tone glide: the CV base combined with a [da] F3 sinusoidal transition of increased amplitude was paired with the CV base combined with a [ga] F3 sinusoidal transition of increased amplitude. Subjects were asked to silently identify each token as being either a [da] with a chirp or a [ga] with a chirp. This condition was intended to provide data about processing a simultaneous speech + nonspeech stimulus.

4. [da] vs. [ga]: the CV base combined with a [ga] F3 sinusoidal transition of equal amplitude was paired with the $\mathrm{CV}$ base combined with a [da] F3 sinusoidal transition of equal amplitude. Subjects were asked to silently identify each token as being either a [da] or a [ga]. This condition was intended to provide data about processing only speech stimuli. 
5. [da] tone glide vs. [ga] tone glide: the [da] F3 sinusoidal transition only was paired with the [ga] sinusoidal transition only, both presented at an increased amplitude. Subjects were asked to silently identify each token as being either [da] or [ga]. This condition was intended to provide data about processing only nonspeech stimuli.

The conditions were presented to each subject in the same order, as listed above, so that subjects would not become exposed to the tone glides as nonspeech tokens prior to their exposure to the tone glides within the context of speech. Within each condition, there were 50 occurrences of the two token types, for a total of 100 tokens presented in pseudorandom order.

In order to control the subject's attention and involvement during testing, a simple behavioral task was included while the ERPs were being recorded: Subjects were to identify each token as it occurred; there was no target stimulus token in any of the conditions. Prior to testing, subjects participated in a training session (which included multiple exposures to all the stimulus tokens within a thirty minute session) in which they were required to correctly identify $80 \%$ of the practice stimuli in order to be included in the experiment. Subjects first were exposed to the different stimulus types. They heard five repetitions of each of the six stimulus types. Then they participated in a training and practice session prior to each test condition. Subjects first heard two each of the two stimulus types to be identified in the upcoming condition, followed by five each of those same two stimulus types, presented in random order, and they were asked to identify each token aloud. Subjects had to obtain a score of $80 \%$ correct to continue with the test, and they could repeat the practice and were given 
feedback until they reached the criterion level (see Appendix A for instruction sheets for each condition). During the actual test, items were presented in a pseudorandom order. Subjects were also instructed to refrain from making any eye movements or blinks or muscle movements during the test portion. To minimize eye movement, subjects were asked to focus on a dot placed on a flat surface at eye level. There was a five minute rest period between conditions. Therefore, the entire testing session took approximately three hours, including set-up with electrodes. A mean of 32 trials out of 50 per subject were averaged to obtain waveforms, with a range of 10 to 49 trials.

Brain electrophysiological responses were obtained from fifteen active gold cup electrodes placed on a subject's scalp, following the International 10-20 convention (Jasper, 1958), at Fz, Cz, Pz, F3, C3, P3, F4, C4, P4, F7, T3, T5, F8, T4, and T6, each referred to linked ears (Figure 6). Fpz on the forehead served as a ground. Eye movements and blinks were monitored via electrodes placed at the right external canthus and above the left supraorbital ridge. The acceptable impedance level was $5 \mathrm{kOhms}$ or below at each electrode. The difference in interelectrode impedance levels was no more than $3 \mathrm{kOhms}$. The electrodes were connected to a 20 -channel electrophysiological data acquisition and analysis system (Brain Atlas, Biologic Systems Corp.), which was used to collect and analyze the data. 


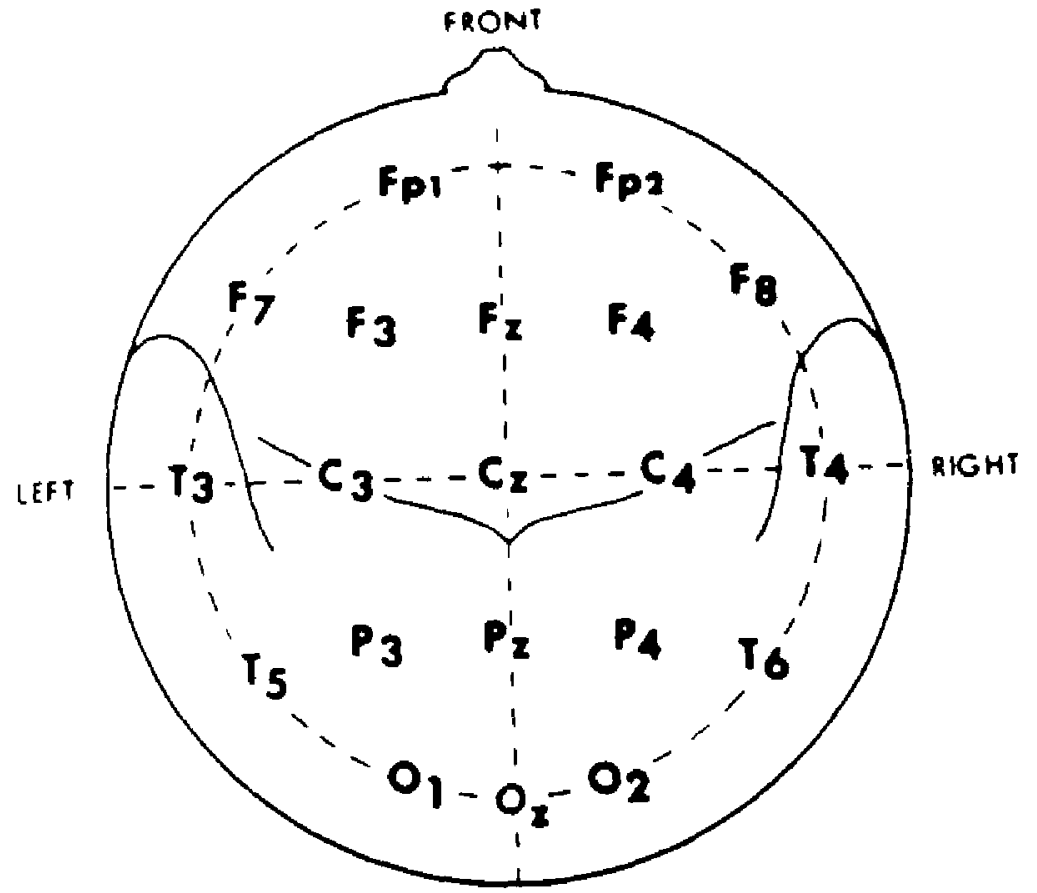

FIGURE 6. International 10-20 System of Electrode Placement. The figure provides the alphanumeric designations of electrode placement on the scalp for EEG recordings. 
Equipment

An AT\&T Personal Computer was used which contained two 12-bit Digital-to-Analog (D/A) boards (DT2801). Having two D/A boards allowed for four output channels, of which three were necessary for this study. One channel outputted the stimulus tokens directly to headphones (TDH-49) binaurally, while two other channels each outputted a 5 volt trigger pulse directly to the Biologic system (Figure 7). The trigger pulse directed the Biologic system to begin ERP collection and to store the data in different memory banks, with all of the data for one stimulus type going to one memory bank, and all of the data for the other stimulus type going to another memory bank. In this way, the incoming raw EEG data for each of the two types of stimulus tokens being presented in one condition could be summed and averaged separately. Brain activity was collected for a $1024 \mathrm{msec}$ epoch of data during the presentation of the stimulus tokens. Collection began $128 \mathrm{msec}$ prior to stimulus onset to obtain a pre-stimulus baseline. A sampling rate of 256 samples per second was used. A bandpass filter was used with a bandwidth .3 to $30 \mathrm{~Hz}$, which filters out signals below $.3 \mathrm{~Hz}$ and above $30 \mathrm{~Hz}$. A $60 \mathrm{~Hz}$ notch filter was in place to remove 60 cycle interference.

\section{Artifact-Handling}

In order to insure that artifacts did not confound the results, several steps were taken to control for them (Torello and McCarley, 1986): 


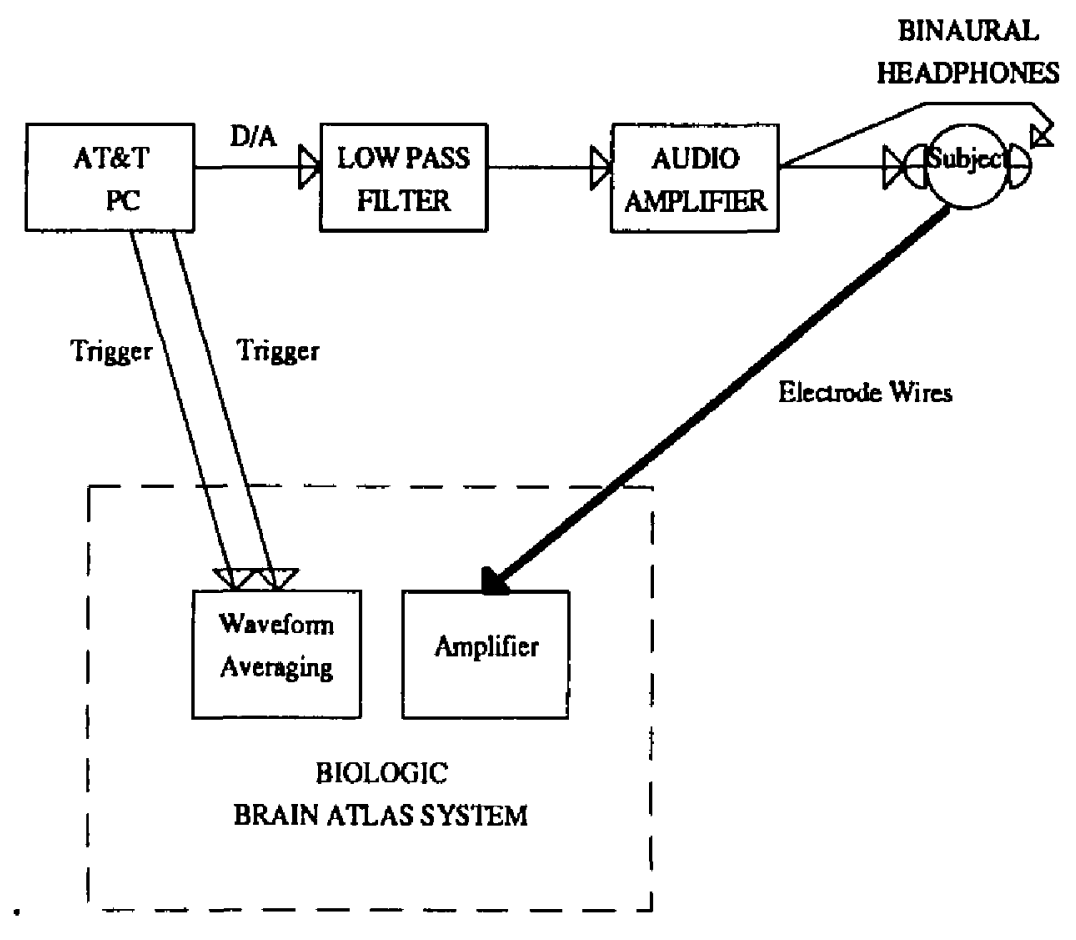

FIGURE 7. Schematic of equipment set-up. 
1. Use of additional electrodes to monitor eye movements, blinks, and muscle activity (i.e., the electrodes around the eyes previously mentioned);

2. Asking subjects to minimize muscle and eye movement during the experiment;

3. Having the experimenter also monitor during the experiment (1) the signals from the additional electrodes and (2) watch subjects for blinks, etc.;

4. Having the computer automatically reject any EEG epoch containing artifact that exceeds $+/-75$ microvolts;

5. Use of the artifact detection electrodes as references during analysis to detect any possible low level artifacts.

Frequent rest periods were provided to facilitate data acquisition. By using these control procedures, it is more likely that the ERP measures reflect relevant brain activity, rather than unrelated extra-cerebral sources (Nunez, 1981).

Waveform Measurement and Analysis

Most analyses utilized subjects as their own controls. For the purposes of this dissertation, only one channel was analyzed, $\mathrm{Cz}$, because typically it has a high signal-to-noise ratio. This is a typical technique used in ERP studies. After consulting the literature, it became evident that there is no previous ERP study which has used the type of stimuli used in this 
study. Therefore, there was no reference for the type of waveforms that might occur. Since an expected family of waveforms was unknown for this type of stimuli, a set of criteria to select and measure the waveforms had to be created. First, three pilot subjects' waveforms were viewed to get an idea of any common patterns that might exist. Then for the subjects used in the experiment, the following criteria for waveform measurement were used: the amplitude values of all peaks in the $128 \mathrm{msec}$ prestimulus baseline were measured from peak-to-peak. These values were multiplied by two. The highest of these values was used as the reference to which all poststimulus peaks were compared. A poststimulus peak was considered to be salient if its baseline-to-peak amplitude was at least twice the value of the prestimulus baseline amplitude, and the peak had to occur in at least $50 \%$ of the subjects. In this study, the salient peaks occurred minimally in $60 \%$ of the subjects, and at least half of the subjects showed all waveform peaks in every condition. The baseline reference value had to be determined separately for each subject, based on each subject's own baseline amplitudes. All relevant waveforms were identified, noting polarity, and measuring baseline-to-peak amplitudes and peak latencies. All waveform measurements were made at the maximum baseline-to-peak excursion. If a maximum excursion amplitude did not change for several milliseconds (a broad peak wave), then the average between the low latency at that level and the high latency at that level was computed. If multiple peaks with the same amplitude occurred, the average between the first peak latency and the last peak latency at that level was computed. All measurements were made by the experimenter. ANOVAs were performed on these data within each 
condition. The first positive peak that passed all the criteria of significance was labelled as P1; the first negative peak as N1. The second positive peak was labeled as $\mathrm{P} 2$, and the second negative peak as $\mathrm{N} 2$, and so on. Waveforms that had no discemible peaks or were contaminated with too many artifacts were discarded. As mentioned before, three subjects were eliminated entirely from the analysis because there was too much artifact ( $50 \%$ or more of the trials had been rejected as artifact), and each salient waveform was seen in at least $60 \%$ of the subjects that were included in the analysis. Prior to measurement, all waveforms first underwent baselinecorrection, which controls for low frequency noise, using a standard software provided by Biologic. The prestimulus period was used as a baseline for this procedure. In addition, each waveform was smoothed one time using a three-point smoothing algorithm (contained in software provided by Biologic), which controls for high frequency noise. Later the Grand Average waveforms were examined and they confirmed that our criteria were appropriate. Figure 8 provides a sample waveform that shows significant measurement points. 


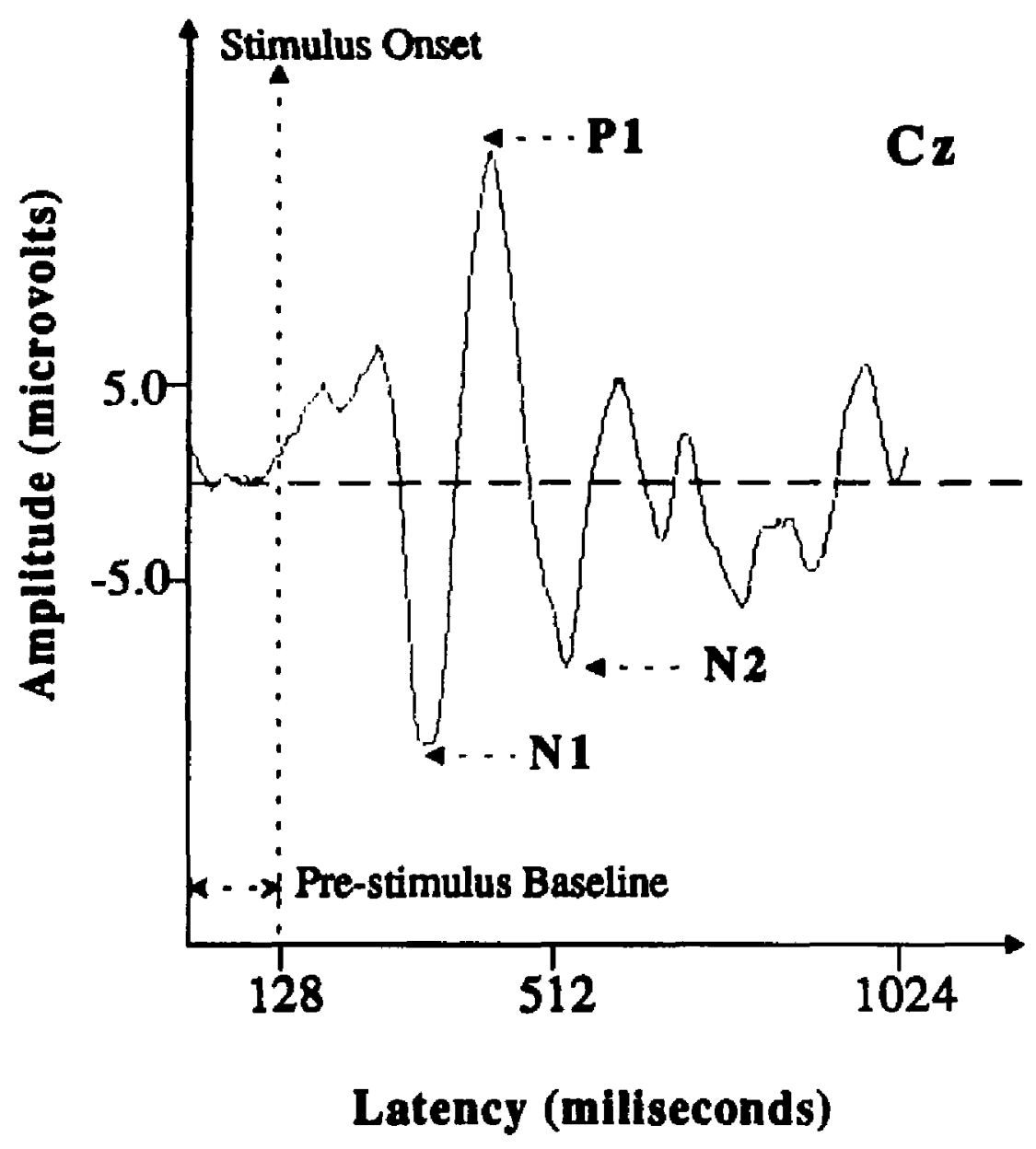

FIGURE 8. Sample waveform referencing significant measurement points used in this study. 


\section{CHAPTER III RESULTS AND DISCUSSION}

For the initial analysis, data from the $\mathrm{Cz}$ electrode only was analyzed, and latency and baseline-to-peak amplitude values were measured. As was explained in the Methodology section, this is a typical technique used in ERP studies because $\mathrm{Cz}$ usually has a high signal-to-noise ratio, and if significant results were not found at this electrode then it would be less likely that significant results would be found at other electrode sites. In subsequent studies analyses of other electrode sites will be performed, from which topographic information can be obtained, but that is beyond the scope of the present dissertation.

For this discussion $N 1, P 1$, and $N 2$ refer to the first negative peak, the first positive peak, and the second negative peak, respectively. They are not intended to refer necessarily to the $\mathrm{N} 100$ (a negative peak occurring generally around $100 \mathrm{msec}$ after a stimulus has been presented), P100 (a positive peak that immediately follows the $\mathrm{N} 100$, occurring generally around $100 \mathrm{msec}$ after a stimulus has been presented), or N200 (a negative peak occurring generally around $200 \mathrm{msec}$ after a stimulus has been presented), as is sometimes done. Since there are no previous studies that have used ERPs to monitor responses to the type of stimuli used in this study, it is difficult to definitively identify the peaks that occur in this data as being one 
of the commonly identified peaks (N100, P100, N200), especially given the current interpretation that some of them represent certain cognitive furctions (N100 - attentiveness, N200-stimulus detection, P100 is usually seen in visual ERPs). The $\mathrm{N} 1$ in this study occurs, on average, around $215 \mathrm{msec}$; the P1, around $302 \mathrm{msec}$; and the N2, around $396 \mathrm{msec}$. These values may be within a range (albeit, at the far end of those ranges) that is acceptable for the N100, P100, and N200, and therefore, the peaks in this study may be related to those peaks; however, more investigation must be done before this claim can be made. Representative waveforms for each type of stimulus within each condition are shown in Appendix B. Figures 9-12 present sample waveforms from two subjects during Condition 1 ([da] vs. [da] + tone glide). Figures 9 and 11 present the waveforms representing the average of the responses to just the plain [da] stimuli for the two subjects. Figures 10 and 12 present the waveforms representing responses to duplex [da] only for the two subjects. Appendix C includes Grand Average waveforms for each stimulus type within each condition.

By viewing the sample waveforms in Figures 9-12, it can be seen that there was considerable variability across subjects, especially in the amplitude values. Latency values were typically more consistent across subjects.

Conditions 1 and 2

In Condition 1 the pure speech token [da] was presented with the duplex token [da] and in Condition 2 the pure speech token [ga] was 


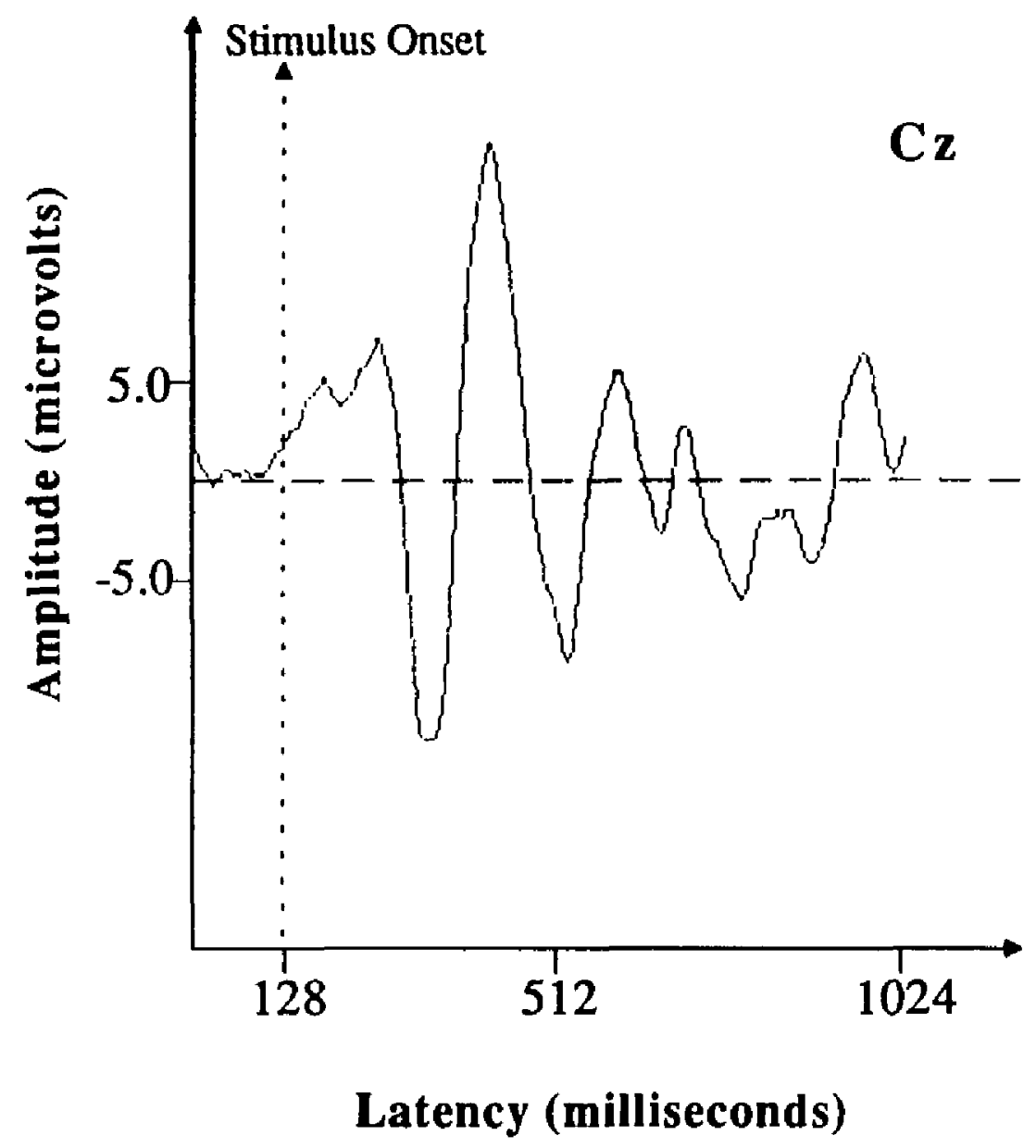

FIGURE 9. Sample waveform from Subject 4. This waveform represents only the responses to plain [da] in Condition 1 (plain [da] vs. duplex [da]). 


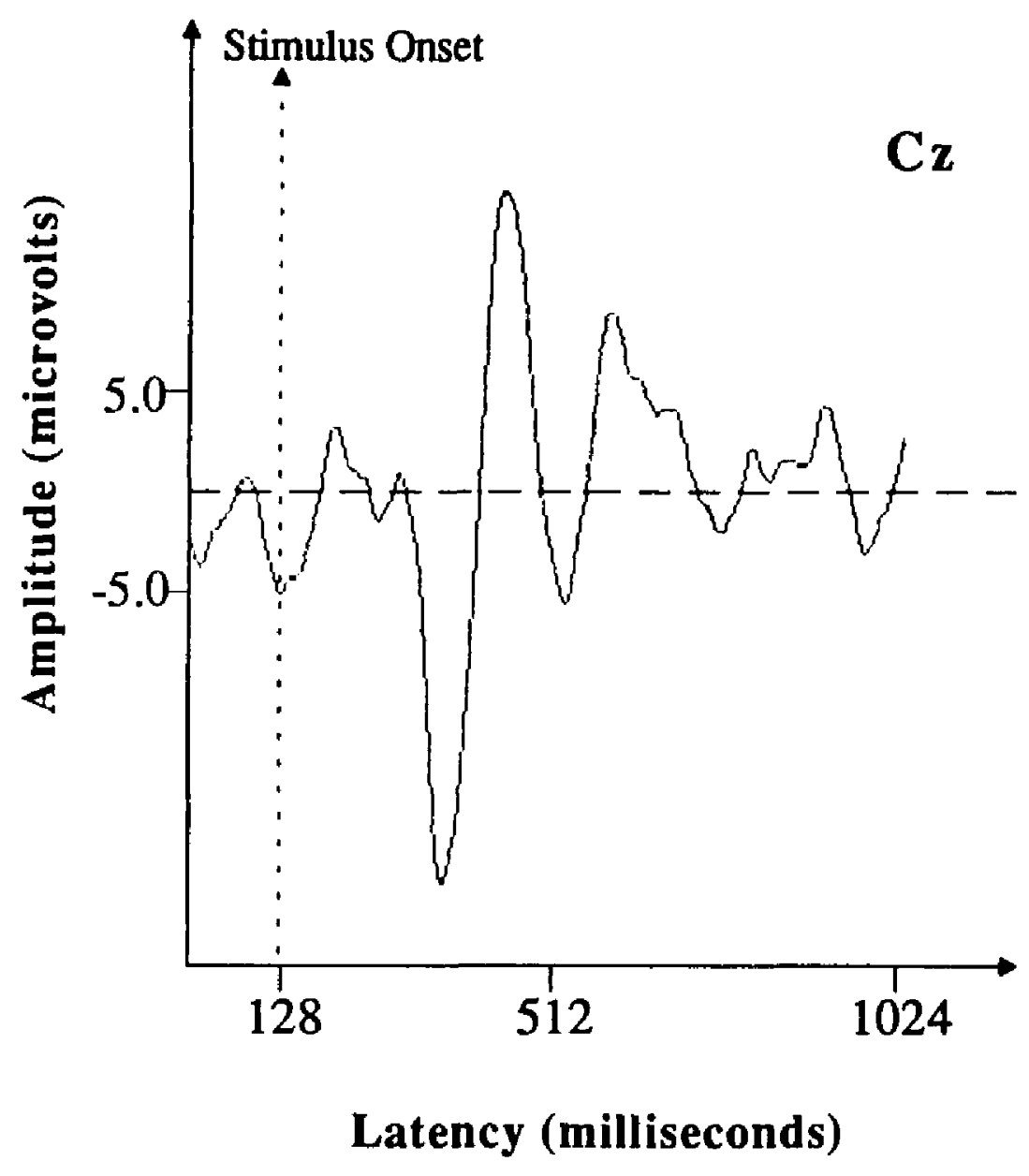

FIGURE 10. Sample waveform from Subject 4. This waveform represents only the responses to duplex [da] in Condition 1 (plain [da] vs. duplex [da]). 


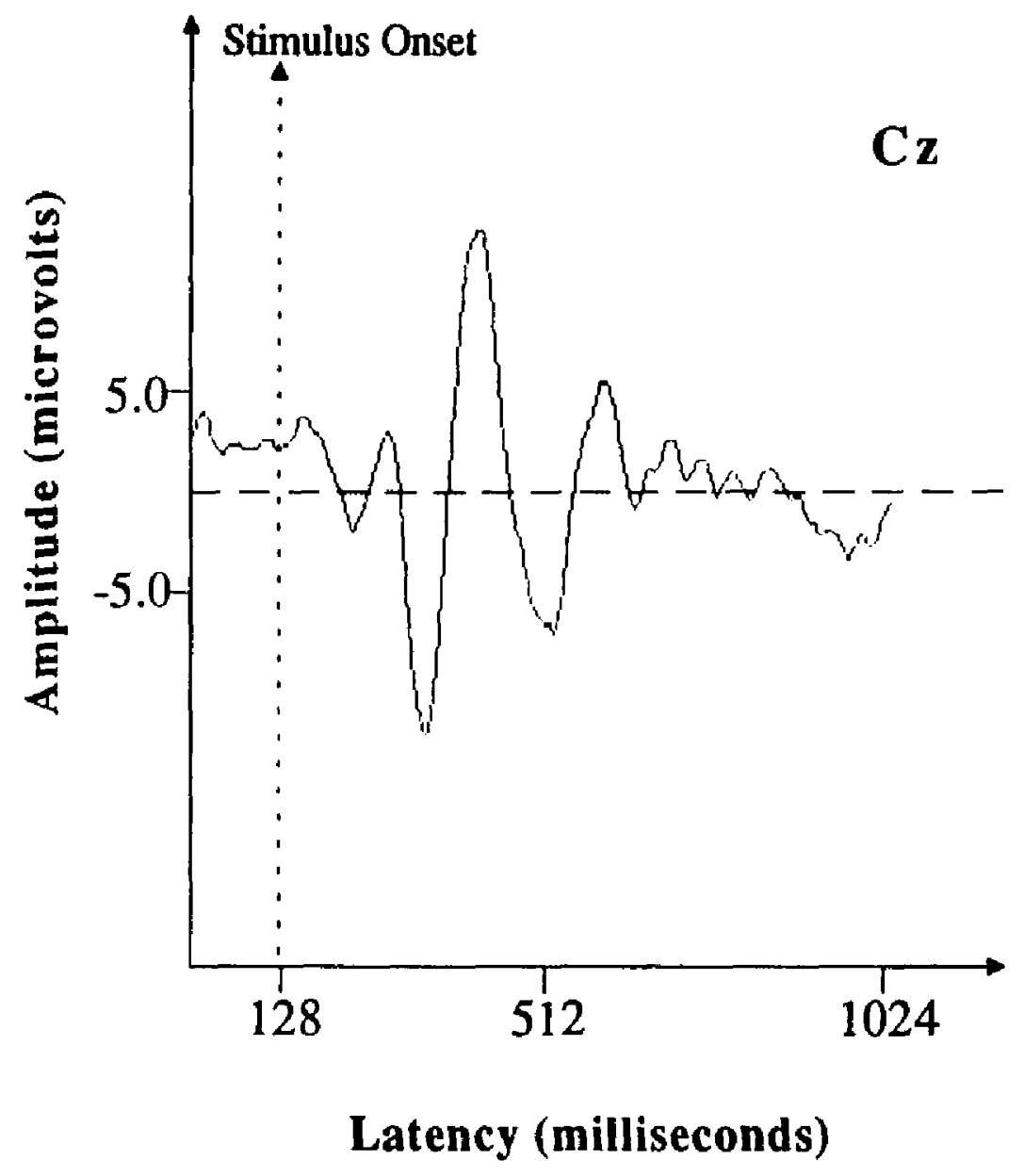

FIGURE 11. Sample waveform from Subject 13. This waveform represents only the responses to plain [da] in Condition 1 (plain [da] vs. duplex [da]). 


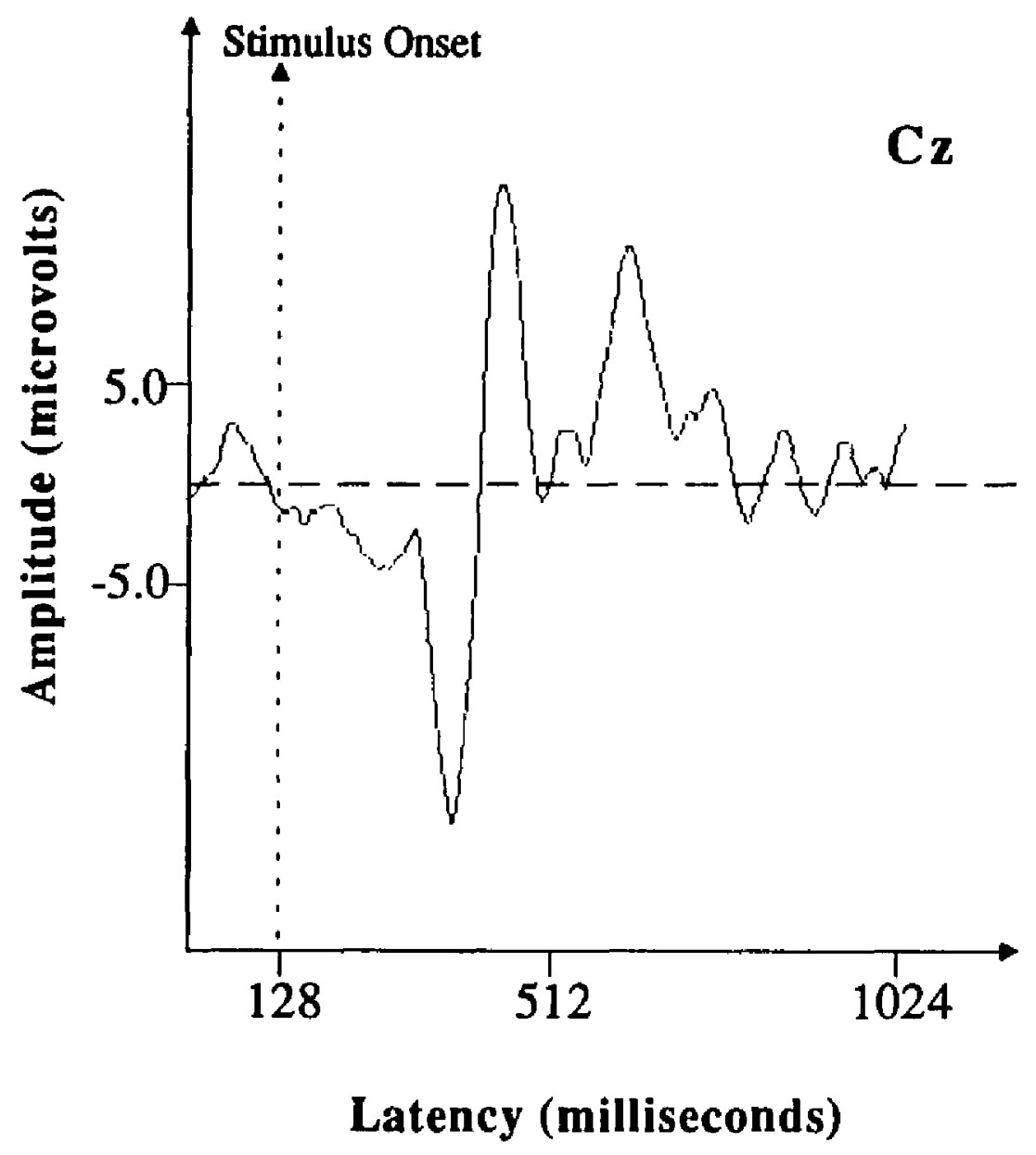

FIGURE 12. Sample waveform from Subject 13. This waveform represents only the responses to duplex [da] in Condition 1 (plain [da] vs. duplex [da]). 
presented with the duplex token [ga]. The subject's task was to silently identify each token as it occurred. These two conditions could be collapsed across each other to perform the analyses since there was no syllable effect, as is discussed below. In general, the duplex stimuli for both [da] and [ga] had significantly longer N1, P1, and N2 latencies than the plain stimuli in Conditions 1 and 2. The mean latencies and standard deviations of $\mathrm{N} 1$ in Conditions 1 and 2 are presented in Table 3. A two-way repeated-measures analysis of variance of the latency values using Geisser-Greenhouse correction with the within-subject factors stimulus type (plain vs. duplex) and syllable ([da] vs. [ga]) showed significant main effects of stimulus type for $\mathrm{N} 1[\mathrm{~F}(1,15)=253.56, \mathrm{p}<.000]$. There was no main effect of syllable for $\mathrm{N} 1[\mathrm{~F}(1,15)=.45, \mathrm{p}<.513]$, nor was there any interaction between stimulus type and syllable $[F(1,15)=.10, p<.754]$.

Table 4 presents the mean latencies and standard deviations of P1 in Conditions 1 and 2. There was a significant main effect of stimulus type for P1 $[F(1,16)=119.09, \mathrm{p}<.000]$. However, the factor syllable was not significant $[F(1,16)=.04, p<.848]$. The interaction between stimulus type and syllable was also not significant $[F(1,16)=.14, p<.713]$.

The N2 component's mean latencies and standard deviations in Conditions 1 and 2 are listed in Table 5. The factor stimulus type was significant for $\mathrm{N} 2[\mathrm{~F}(1,14)=15.40, \mathrm{p}<.002]$. There was no main effect of syllable $[F(1,14)=2.00, p<.179)$. The interaction effect between stimulus type and syllable was marginally significant $[F(1,14)=3.86, p<.069]$.

The longer latencies that occurred for the duplex stimuli across all measures (N1, P1, N2) in Conditions 1 and 2 indicate that additional 
TABLE 3. Mean latency values (in msec) and standard deviations of $\mathrm{N} 1$ across subjects in Conditions 1 and 2

\begin{tabular}{cccc}
\hline & \multicolumn{3}{c}{ Stimulus Type } \\
\cline { 2 - 4 } Syllable & Plain & Duplex & Mean \\
\hline [da] & $202(10.5)$ & $234(9.2)$ & $218(9.9)$ \\
[ga] & $204(11.5)$ & $234(8.3)$ & $219(10.1)$ \\
Mean & $203(11.0)$ & $234(8.8)$ \\
\hline
\end{tabular}


TABLE 4. Mean latency values (in msec) and standard deviations of Pl across subjects in Conditions 1 and 2

\begin{tabular}{cccc}
\hline & \multicolumn{3}{c}{ Stimulus Type } \\
\cline { 2 - 4 } Syllable & Plain & Duplex & Mean \\
\hline [da] & $294(10.6)$ & $319(11.6)$ & $307(11.1)$ \\
[ga] & $292(12.7)$ & $319(9.3)$ & $306.5(11.0)$ \\
Mean & $293(11.7)$ & $319(10.5)$ \\
\hline
\end{tabular}


TABLE 5. Mean latency values (in msec) and standard deviations of N2 across subjects in Conditions 1 and 2

\begin{tabular}{cccc}
\hline & \multicolumn{3}{c}{ Stimulus Type } \\
\cline { 2 - 4 } Syllable & Plain & Duplex & Mean \\
\hline [da] & $393(29.3)$ & $404(20.4)$ & $398.5(24.9)$ \\
[ga] & $378(16.1)$ & $407(19.9)$ & $392.5(18.0)$ \\
Mean & $387.5(22.7)$ & $404.5(20.2)$ \\
\hline
\end{tabular}


processing of some kind is involved when there is both a speech and a nonspeech component within a stimulus. There are a number of ways that these results may be interpreted. It may be that the speech portion of the stimulus is processed first, and the remaining nonspeech portion of the signal is then processed, perhaps by a different module from that applied to the speech portion, as suggested by Whalen and Liberman (1987). Another interpretation may be that there is, in fact, two different modules in which the different portions of the duplex stimulus are processed, as suggested by Liberman and Mattingly (1985). Because the signal must travel to these two modules or just by having to be processed in two separate modules, latency might be increased.

In general, amplitude values in Conditions 1 and 2 reflected some effects of stimulus type and syllable: N1 and P1 amplitude values were larger for the duplex stimuli than for the plain stimuli, and the P1 amplitude value was larger for [da] than for [ga]. Mean amplitudes and standard deviations for $\mathrm{N} 1$ across subjects in Conditions 1 and 2 are shown in Table 6. A two-way repeated-measures analysis of variance of the amplitude values using Geisser-Greenhouse correction with the within-subject factors stimulus type and syllable showed significant main effects of stimulus type for $\mathrm{N} 1[\mathrm{~F}(1,13)=5.10, \mathrm{p}<.042]$. There were no significant differences between amplitude values for [da] and [ga], $[\mathrm{F}(1,13)=2.09, \mathrm{p}<$ $.172]$, nor was there any interaction between stimulus type and syllable $[F(1$, 13) $=.29, \mathrm{p}<.602]$ 
TABLE 6. Mean amplitude values (in $\mu \mathrm{V}$ ) and standard deviations of $\mathrm{N} 1$ across subjects in Conditions 1 and 2

\begin{tabular}{cccc}
\hline & \multicolumn{3}{c}{ Stimulus Type } \\
\cline { 2 - 4 } Syllable & Plain & Duplex & Mean \\
\hline$[\mathrm{da}]$ & $-11.7(4.6)$ & $-13.8(4.7)$ & $-12.75(4.65)$ \\
{$[\mathrm{ga}]$} & $-11.2(4.9)$ & $-12.6(5.5)$ & $-11.9(5.2)$ \\
Mean & $-11.45(4.75)$ & $-13.2(5.1)$ \\
\hline
\end{tabular}


In Table 7, mean amplitude values and standard deviations of P1 in Conditions 1 and 2 are presented. Stimulus type was also significant for P1 $[F(1,15)=5.29, p<.036] . P 1$ was the only peak that showed an effect of syllable $[F(1,15)=21.89, p<.000]$. No interaction effect was apparent $[F(1,15)=2.71, \mathrm{p}<.121]$.

N2 mean amplitudes and standard deviations for Conditions 1 and 2 can be seen in Table 8. N2 showed no significant difference in stimulus type $[\mathrm{F}(1,11)=1.46, \mathrm{p}<.252] . \quad \mathrm{N} 2$ amplitude values were not significantly different between $[\mathrm{da}]$ and $[\mathrm{ga}],[F(1,11)=.00, \mathrm{p}<.960]$, and there were no significant interaction effects between stimulus type and syllable $[\mathrm{F}(1,11)=.05, \mathrm{p}<.828]$.

Overall, the amplitude did not differ significantly between the two stimulus types. The peaks which showed significant differences (N1 and P1 for stimulus type, and P1 for syllable) are early components and may be a reflection of the subject's attention to the stimuli, as early components have sometimes been interpreted (Picton and Stuss, 1984).

In Conditions 1 and 2, the subject's task was to identify the occurrence of a tone glide (a nonspeech stimulus) within the context of a speech stimulus. Therefore, the stimulus type provided information necessary to making identification of a tone glide possible while the syllable did not provide such information. The results indicate that more processing was required at all measures (N1, P1, and N2) for the stimuli that contained both the speech and nonspeech components. The larger amplitude of the earlier peaks for the duplex tokens may be because the increased intensity of the F3 transition made them more noticeable to the subject. 
TABLE 7. Mean amplitude values (in $\mu \mathrm{V}$ ) and standard deviations of $\mathrm{P} 1$ across subjects in Conditions 1 and 2

\begin{tabular}{cccc}
\hline & \multicolumn{3}{c}{ Stimulus Type } \\
\cline { 2 - 4 } Syllable & Plain & Duplex & Mean \\
\hline [da] & $12.2(3.7)$ & $15.0(6.4)$ & $13.6(5.1)$ \\
[ga] & $9.8(3.4)$ & $10.4(2.9)$ & $10.1 \quad(3.2)$ \\
Mean & $11.0(3.55)$ & $12.7(4.7)$ & \\
\hline
\end{tabular}


TABLE 8. Mean amplitude values (in $\mu \mathrm{V}$ ) and standard deviations of $\mathrm{N} 2$ across subjects in Conditions 1 and 2

\begin{tabular}{cccc}
\hline & \multicolumn{3}{c}{ Stimulus Type } \\
\cline { 2 - 4 } Syllable & Plain & Duplex & Mean \\
\hline [da] & $-7.0(4.3)$ & $-5.8(4.3)$ & $-6.4(4.3)$ \\
[ga] & $-7.1(3.8)$ & $-5.7(3.0)$ & $-6.4(3.4)$ \\
Mean & $-7.05(4.1)$ & $-5.75(3.7)$ \\
\hline
\end{tabular}


Conditions 3 and 4

In Condition 3 subjects listened to the two duplex tokens (duplex [da] vs. duplex [ga]) and were to silently each token as it occurred. In Condition 4 subjects listened to the two plain speech tokens (plain [da] vs. plain [ga]) and were to silently identify each token as it occurred. Conditions 3 and 4 could be collapsed across conditions for analysis because stimulus type was not significant, as is discussed below. Generally, latency was longer for [ga], regardless of the stimulus type. Table 9 presents the N1 mean latency values and standard deviations in Conditions 3 and 4. A two-way repeatedmeasures analysis of variance of the latency values using GeisserGreenhouse correction with the within-subject factors stimulus type and syllable revealed significant main effects of syllable for $\mathrm{N} 1[\mathrm{~F}(1,11)=$ $118.22, \mathrm{p}<.000]$, but not of stimulus type $[\mathrm{F}(1,11)=2.01, \mathrm{p}<.184]$. There were no interaction effects between syllable and stimulus type $[F(1,11)=$ $.10, \mathrm{p}<.757]$.

Table 10 shows the mean latencies and standard deviations for $\mathrm{P} 1$ in Conditions 3 and 4. Syllable was significant for $P 1[F(1,11)=168.02, p<$ $.000]$. Stimulus type was not significant $[F(1,11)=.68, p<.427]$, nor was there any interaction between syllable and stimulus type $[F(1,11)=.00, p<$ $1.00]$.

The mean latency values and standard deviations of $\mathrm{N} 2$ in Conditions 3 and 4 can be seen in Table 11. N2 showed a significant main effect of syllable $[F(1,7)=23.90, p<.002]$, and of stimulus type $[F(1,7)=7.32, p<$ .030]. There was no interaction effect $[F(1,7)=2.45, p<.161]$. 
TABLE 9. Mean latency values (in msec) and standard deviations of $\mathrm{N} 1$ across subjects in Conditions 3 and 4.

\begin{tabular}{cccc}
\hline & \multicolumn{3}{c}{ Stimulus Type } \\
\cline { 2 - 4 } Syllable & Plain & Duplex & Mean \\
\hline [da] & $198(8.4)$ & $201(10.7)$ & $199.5(9.6)$ \\
[ga] & $231(11.1)$ & $234(11.0)$ & $232.5(11.15)$ \\
Mean & $214.5(9.8)$ & $217.5(10.9)$ \\
\hline
\end{tabular}


TABLE 10. Mean latency values (in msec) and standard deviations of $P 1$ across subjects in Conditions 3 and 4.

\begin{tabular}{cccc}
\hline & \multicolumn{3}{c}{ Stimulus Type } \\
\cline { 2 - 4 } Syllable & Plain & Duplex & Mean \\
\hline$[\mathrm{da}]$ & $288(10.0)$ & $290(13.8)$ & $289(11.9)$ \\
{$[\mathrm{ga}]$} & $316(12.9)$ & $319(8.6)$ & $317.5(10.5)$ \\
Mean & $302(11.5)$ & $304.5(11.2)$ \\
\end{tabular}


TABLE 11. Mean latency values (in msec) and standard deviations of $\mathrm{N} 2$ actoss subjects in Conditions 3 and 4.

\begin{tabular}{cccc}
\hline & \multicolumn{3}{c}{ Stimulus Type } \\
\cline { 2 - 4 } Syllable & Plain & Duplex & Mean \\
\hline [da] & $372(23.0)$ & $383(17.1)$ & $377.5(20.1)$ \\
[ga] & $403(25.0)$ & $441(56.6)$ & $422(40.8)$ \\
Mean & $387.5(24.0)$ & $412(36.9)$ \\
\end{tabular}


In Conditions 3 and 4, when the same stimulus types were paired together, with the task being to identify a given phoneme, no effect of stimulus type occurred; only a syllable effect was seen. Such a result is not surprising since the only difference between the two stimulus tokens was the initial phoneme. Latency values were consistently longer for [ga] than [da], indicating that more analysis is required for [ga].

In Conditions 3 and 4, amplitude values of $\mathrm{N} 1$ only were larger for [da] and for duplex stimuli. The mean amplitude values and standard deviations of N1 in Conditions 3 and 4 are listed in Table 12. A two-way repeated-measures analysis of variance of amplitude values using GeisserGreenhouse correction with the within-subject factors syllable and stimulus type indicated a significant main effect of syllable for $N 1[F(1,10)=7.25, p$ $<.023]$. A main effect of stimulus type was evident in $\mathrm{N} 1$ only $[\mathrm{F}(1,10)=$ $10.76, p<.008]$. No interaction occurred between syllable and stimulus type $[F(1,10)=.11, \mathrm{p}<.747]$.

Table 13 presents the mean amplitudes and standard deviations of $P 1$ in Conditions 3 and 4. Differences in amplitude values across syllables and stimulus type were not significant for $\mathrm{P} 1[\mathrm{~F}(1,10)=.10, \mathrm{p}<.754]$ and $[\mathrm{F}(1$, $10)=.03, p<.864]$, respectively. No interaction effect occurred between syllable and stimulus type $[\mathrm{F}(1,10)=.01, \mathrm{p}<.944]$.

In Table 14, the mean amplitudes and standard deviations of $\mathrm{N} 2$ are shown. Neither syllable nor stimulus type were significant for $N 2[F(1,6)=$ $1.68, \mathrm{p}<.242],[\mathrm{F}(1,6)=1.68, \mathrm{p}<.242]$, respectively, nor was there any interaction effect $[F(1,6)=.43, p<.538]$. 
TABLE 12. Mean amplitude values (in msec) and standard deviations of $\mathrm{N} 1$ across subjects in Conditions 3 and 4.

\begin{tabular}{cccc}
\hline & \multicolumn{3}{c}{ Stimulus Type } \\
\cline { 2 - 4 } Syllable & Plain & Duplex & Mean \\
\hline [da] & $-13.5(5.0)$ & $-10.6(4.0)$ & $-12.05(4.5)$ \\
[ga] & $-11.5(5.1)$ & $-9.0(4.1)$ & $-10.25(4.6)$ \\
Mean & $-12.5(5.05)$ & $-9.8(4.05)$ \\
\end{tabular}


TABLE 13. Mean amplitude values (in $\mathrm{msec}$ ) and standard deviations of $P 1$ across subjects in Conditions 3 and 4.

\begin{tabular}{cccc}
\hline & \multicolumn{3}{c}{ Stimulus Type } \\
\cline { 2 - 4 } Syllable & Plain & Duplex & Mean \\
\hline [da] & $11.4(4.1)$ & $11.3(3.3)$ & $11.35(3.7)$ \\
[ga] & $11.2(4.9)$ & $11.0(3.7)$ & $11.1(4.3)$ \\
Mean & $11.3(4.5)$ & $11.15(3.5)$ \\
\end{tabular}


TABLE 14. Mean amplitude values (in msec) and standard deviations of $\mathrm{N} 2$ across subjects in Conditions 3 and 4.

\begin{tabular}{cccc}
\hline & \multicolumn{3}{c}{ Stimulus Type } \\
\cline { 2 - 4 } Syllable & Plain & Duplex & Mean \\
\hline [da] & $-5.4(3.4)$ & $-6.0(3.3)$ & $-5.7(3.35)$ \\
[ga] & $-7.1(1.9)$ & $-6.0(2.2)$ & $-6.55(2.1)$ \\
Mean & $-6.3(2.7)$ & $-6.0(2.8)$ & \\
\hline
\end{tabular}


As in Conditions 1 and 2 , the differences in amplitude values in Conditions 3 and 4 were largely insignificant. Amplitude values of N1 were significantly larger for [da] and for duplex stimuli. The N1 component may reflect the increased saliency of these stimuli (see discussion below regarding the possible reduced saliency of [ga]), such that subjects noticed them more than the [ga] and the plain stimuli.

In Conditions 3 and 4 the task was to identify the token as being either a [da] or a [ga], a purely linguistic task. In Condition 3 both speech and nonspeech components were present. In Condition 4 only the speech component was present. The syllable [ga] had consistently longer latencies than [da], indicating more processing occurring for [ga]. This may be due to the manner in which the [ga] and [da] were synthesized. Both tokens were created without a release burst and it may be that the release burst is a more salient percept for identification purposes for [ga] than it is for [da]. Behaviorally, the majority of subjects reported more difficulty identifying [ga] than [da].

N2 latencies in Conditions 3 and 4 were significantly longer for plain tokens than for duplex tokens. At first this may seem to contradict previous results obtained in Conditions 1 and 2 where the duplex tokens had longer latencies than the plain tokens; however, this result is actually what should be expected. The syllable contained the information necessary to identify the target stimulus in both conditions; however, it may be that stimulus type had some effect on identification. Specifically, the increased amplitude of the F3 transition may have made the syllables in the duplex condition more salient than in the plain condition so that subjects identified target stimuli 
more quickly in the duplex condition. In essence, subjects were receiving information about the stimulus simultaneously from two sources in the duplex condition: the speech component and the nonspeech component. More information may have lead to quicker processing. Similarly, the amplitude of N1 may be larger for duplex stimuli because the increased amplitude of the F3 transition makes the duplex stimuli more salient. The N1 amplitude values were also larger for [da] than [ga]. As mentioned previously, the $\mathrm{Nl}$ may reflect the subject's notice paid to the more salient stimulus token ([da]).

Condition 5

In Condition 5 the two isolated tone glides with different contours were presented (a descending sinusoidal tone glide [da] vs. a rising sinusoidal tone glide [ga]). Subjects were to silently make note when they heard either a [da] or [ga] (At first, subjects were very confused and concerned because they reported that they could not hear anything like a [da] or a [ga]. Finally, it became necessary to instruct subjects simply to make a mental note if they heard anything that sounded like a [da] or a [ga], but that it was OK if they did not hear either one.) Latency values were longer for the [ga] tone glide and amplitude values had a tendency to be larger for the [da] tone glide. Table 15 shows the mean latency values and standard deviation scores for all of the components: $\mathrm{N} 1, \mathrm{P} 1$, and $\mathrm{N} 2$, in Condition 5. A one-way analysis of variance of the latency values using Geisser-Greenhouse correction with the within-subject factor tone contour 
showed a significant main effect of tone contour for all measures: $N 1[F(1$, $12)=374.53, \mathrm{p}<.000], \mathrm{P} 1[\mathrm{~F}(1,14)=64.23, \mathrm{p}<.000]$, and $\mathrm{N} 2[\mathrm{~F}(1,10)=$ $45.21, \mathrm{p}<.000]$.

The mean amplitude values and standard deviations of $\mathrm{N} 1, \mathrm{P} 1$, and $\mathrm{N} 2$ in Condition 5 can be seen in Table 16. A one-way analysis of variance of amplitude values using Geisser-Greenhouse correction with the withinsubject factor syllable revealed a significant main effect of syllable for P1 $[\mathrm{F}(1,14)=4.52, \mathrm{p}<.052]$, a marginally significant effect for $\mathrm{N} 1[\mathrm{~F}(1,12)=$ $4.15, \mathrm{p}<.064]$, and no effect for $\mathrm{N} 2[\mathrm{~F}(1,10)=.28, \mathrm{p}<.606]$.

A tone contour effect is not unexpected for the tone glides because the two tone glides are auditorally distinct and it is probable that subjects assigned, at random, a label to the tone glides in order to more easily make note of their occurrence. This result is consistent with those obtained by Whalen and Liberman (1987), in which their subjects reliably labeled the tone glides as being either [da] or [ga], but their overall accuracy was at chance level. About half the subjects were consistently right and the other half were consistently wrong in Whalen and Liberman's study, and these results were mirrored in the current study.

The fact that the latencies for tone glide [ga] were consistently longer than those for tone glide [da] may be because there was a greater extent of frequency change over time for the tone glide [ga] than for the tone glide [da]. The tone glide for [ga] began at $2175 \mathrm{~Hz}$ and moved to $2600 \mathrm{~Hz}$, a change of $425 \mathrm{~Hz}$ within $50 \mathrm{msec}$. The tone glide for [da] began at $2800 \mathrm{~Hz}$ and moved to $2600 \mathrm{~Hz}$, a change of $200 \mathrm{~Hz}$ within $50 \mathrm{msec}$. Subjects may have needed more time to resolve the greater change of the tone glide [ga], 
resulting in longer latencies for this stimulus token. If this is the case, it may be that this is also a factor affecting the latencies for the duplex [ga] and plain [ga] in Conditions 3 and 4, respectively, which were longer than the latencies for the corresponding [da] tokens. 
TABLE 15. Mean latency values (in msec) and standard deviations of N1, P1, and N2 across subjects in Condition 5.

\begin{tabular}{cccc}
\hline & \multicolumn{3}{c}{ Tone Contour } \\
\cline { 2 - 4 } Syllable & Tone glide [da] & Tone glide [ga] & Mean \\
\hline N1 & $189(10.1)$ & $222(11.4)$ & $205.5(10.8)$ \\
P1 & $273(20.1)$ & $308(16.7)$ & $290.5(18.4)$ \\
N2 & $368(15.1)$ & $407(14.1)$ & $387.5(14.6)$ \\
\hline
\end{tabular}


TABLE 16. Mean amplitude values (in $\mu \mathrm{V}$ ) and standard deviations of $\mathrm{N} 1, \mathrm{P} 1$, and N2 across subjects in Condition 5.

\begin{tabular}{clcr}
\hline & \multicolumn{3}{c}{ Tone Contour } \\
\cline { 2 - 4 } Syllable & Tone glide [da] & Tone glide [ga] & Mean \\
\hline $\mathrm{N} 1$ & $-11.8(2.9)$ & $-9.2(4.0)$ & $-10.5(3.5)$ \\
$\mathrm{P} 1$ & $13.5(7.0)$ & $11.3(6.3)$ & $12.4(6.7)$ \\
$\mathrm{N} 2$ & $-3.9(4.5)$ & $-4.7(2.7)$ & $-4.3(3.6)$ \\
\hline
\end{tabular}




\section{CHAPTER IV \\ CONCLUSIONS AND FUTURE DIRECTIONS}

The intent of this study was to search for neurological evidence that supports the existence of separate cognitive processing modules for linguistic and nonlinguistic auditory stimuli. By using carefully synthesized tokens intended to evoke perception of duplex stimuli, more control was exerted over any extraneous components in a stimulus to which a subject could respond. The speech and nonspeech tokens were completely matched in terms of acoustic characteristics, since the nonspeech tone glide was simply the third formant transition contained in the associated speech token, only with increased amplitude. This allows for more objective determination of differences based on true linguistic characteristics of the stimuli.

In fact, this study did demonstrate that the neural activity in response to the stimuli, as measured by the waveform peaks, was different for the two stimulus types. In Hypothesis 1A, it was postulated that, when pure speech tokens are compared with duplex tokens, the pure speech tokens would have shorter latencies because they have only a linguistic portion to be analyzed. In this study, the peaks representing the neural activity in response to the stimuli containing both the linguistic and nonlinguistic components had significantly longer latencies than did the stimuli containing a linguistic 
component only. One interpretation of this result was discussed previously, that of Whalen and Liberman's (1987) in which stimulus tokens which contain both speech and nonspeech components will take longer to process than those containing only a speech component because linguistic components will be processed first, and then any remaining nonlinguistic components will be processed. A stimulus containing just a speech component will not require any additional time for processing a nonspeech component.

Thus these results appear to support Whalen and Liberman's (1987) hypothesis that the phonetic module first utilizes the information it needs to identify the speech component and after that processing is completed, the remaining "extra" nonspeech information is then processed by the general auditory module. Subjects did not report hearing a tone glide, that is, they heard only a CV syllable, until the tone glide's amplitude was increased.

Another interpretation of this result could be that a duplex stimulus takes longer to process because its linguistic and nonlinguistic components have two different areas in the brain to which they must go for processing, and coordinating these two processing sources in order to make an identification of a stimulus takes longer. This would be consistent with the Motor Theory of speech perception's contention of separate modules for phonetic and auditory stimuli. It could be argued that there is only a single area which processes all auditory information, and that the duplex stimuli had more components than the speech only stimuli, thereby taking longer to process. However, I would argue that this is not the case because the pure speech and the duplex stimuli contained identical components and were 
equal in complexity. Only one acoustic parameter varied: the amplitude of the F3 transition.

Another interpretation could be that there is only one module but that it works at different rates for different stimuli. Certainly some studies lend credence to this hypothesis (cf. Wall, et al., 1991) in that speech tokens had longer latencies than nonspeech tokens. Topographical information can provide a better perspective about this view, and this information will be obtained in a follow-up study.

Yet another possible explanation of these results follows Fowler and Rosenblum's (in press) hypothesis that the apparent differential processing of speech duplex perception tokens is not special to speech but is part of a global process in which a component provides information that makes another signal coherent, as the evidence from their slamming metal door duplex perception experiment suggests.

Hypothesis 1B and 1C predict that when duplex tokens are compared with each other and pure speech tokens are compared with each other, respectively, the peak latencies should be relatively equal since the stimulus types are the same and that any differences would likely be a reflection of the different initial consonants. The results indicate that there generally was no main effect of stimulus type, but a strong effect of syllable. The syllable [ga] had longer latencies than [da] regardless of whether it was the plain or duplex [ga]. As was discussed previously, this result may be due to the characteristics of [ga] that are necessary to make it salient, which may not have been included in the synthesized token. However, the important point is the fact that the stimulus type was generally not significant when the same 
stimulus type was compared, and this supports the results obtained in Conditions 1 and 2, which showed a difference when two different stimulus types were compared.

Hypothesis 1D predicted that when the two tone glides are compared with each other, the only effect (if any) should be related to the different tone contours, and, in fact, that is what happened. Subjects were able to differentiate one tone glide from the other; however, they did not consistently identify either tone glide as being associated with their respective consonants. The longer latencies that occurred for the [ga] tone glide than for the [da] tone glide may have been due to the greater amount of change in the [ga] tone glide token which the subjects had to process, as was discussed previously.

It should be noted that the latency values were fairly consistent across subjects. In general, the latency results support the contention of the existence of separate processing modules for phonetic and auditory signals.

Another way in which the results could be viewed is in regard to the task requirements, which were different among the conditions. In Conditions 1 and 2, subjects had a largely nonlinguistic task: to identify tokens which contained a "chirp." In Conditions 3 and 4, subjects had a largely linguistic task: to identify [da] tokens. In Condition 5, subjects also had a linguistic task: to identify the tone glides as either a [da] or [ga]. These task differences were reflected in the results: for Conditions 1 and 2, the factor stimulus type was significant in terms of latency values for all ERP components and syllable was insignificant, while the opposite was true 
for Conditions 3, 4, and 5 (tone contour was significant in the latter condition).

Hypothesis 2 postulated that there would be a difference in the amplitude values between the speech and nonspeech stimuli, but a speculation of the direction of that difference was not made due to unclear interpretations of amplitude differences. First of all, the results of the study indicate that amplitude was highly variable among subjects (see sample waveforms in Figures 9-12, and in Appendix B). Amplitude differences among the stimuli were mostly insignificant, except for some early peaks which probably indicate subjects' notice of a target stimulus. If the assumption is made that amplitude reflects the amount of cell activation involved in a given process, then this result is not surprising. There is no indication that the processing of one type of auditory stimulus, be it speech or nonspeech, involves any more or less neural activity than another.

It should also be noted that no particular ERP component seemed to differentiate speech from nonspeech stimuli either in terms of amplitude or latency. Rather, the pattern of the waveform, especially in terms of the latencies, tended to be consistent for each component (N1, P1, and N2). Thus if the N1 latency was longer for a given condition, the latencies for P1 and $\mathrm{N} 2$ also tended to be longer in that condition.

In the Introduction of this dissertation, it was stated that one of the goals of this study was to prepare for future investigations by recording electrical activity from a full set of electrode sites. These recordings were taken, which provides a direction for future research. Future research will concentrate on analyzing other electrode placements to gain information 
regarding the topographical distribution of the two types of stimuli. Such an analysis should provide insight as to the locations of the two modules, or at least, information about the distribution of neural activity. The different electrode sites will be analyzed for the amount of neural activity that is occurring in an area at a given time and compared to each other. If different neural areas show different levels of activity following the stimulus presentation, then some speculations may be made regarding the location of processing centers for a particular stimulus type.

In addition, it would further strengthen the specialized phonetic module hypothesis if a similar study were done, only utilizing nonspeech stimuli to evoke a duplex percept, as Fowler and Rosenblum (in press) have done with the sounds of a slamming metal door vs. a slamming wooden door. As you may recall, Fowler and Rosenblum found a similar duplex perception effect with these nonspeech stimuli and contend, therefore, that this effect does not reflect a special phonetic module. They suggest that duplex perception, especially processing precedence, is the result of a more global process. Specifically, processing precedence occurs when a chirp provides critical missing information in a simultaneously presented acoustic signal so that together the two parts of the signal specify some soundproducing natural event in the environment. The remainder, after the missing information is provided, is heard as a separate signal. If a study were done in which responses to nonspeech duplex perception stimuli were monitored by ERPs and no significant differences were found in the waveforms of nonspeech duplex stimuli, then it would provide convincing evidence that a specialized phonetic module exists. 
APPENDIX A

INSTRUCTIONS GIVEN TO SUBJECTS PRIOR TO EACH CONDITION 


\section{DUPLEX PERCEPTION STUDY}

Instructions

When testing begins, remain as still as possible, that is, don't move any muscles in your body while a test set is being run. Relax and try not to tense up your muscles. Breathe normally. In front of you is a yellow dot. Stare at that dot throughout each test set and try not to blink or move your eyes.

1. In this condition, either a plain [da] or a [da] + tone glide will be presented. Please listen and silently identify which one you hear.

2. In this condition, either a plain [ga] or a [ga] + tone glide will be presented. Please listen and identify which one you hear.

3. Now either a [da] + tone glide or a [ga] + tone glide will be presented. Please listen and silently identify which one you hear.

4. Finally, either a plain [da] or a plain [ga] will be presented. Please listen and silently identify which one you hear.

5. Two different tone glides will be presented. Please listen and silently identify these as being either [da] or [ga]. 


\section{APPENDIX B}

REPRESENTATIVE ERP WAVEFORMS OF THREE SUBJECTS FOR EACH STIMULUS WITHIN EACH CONDITION 


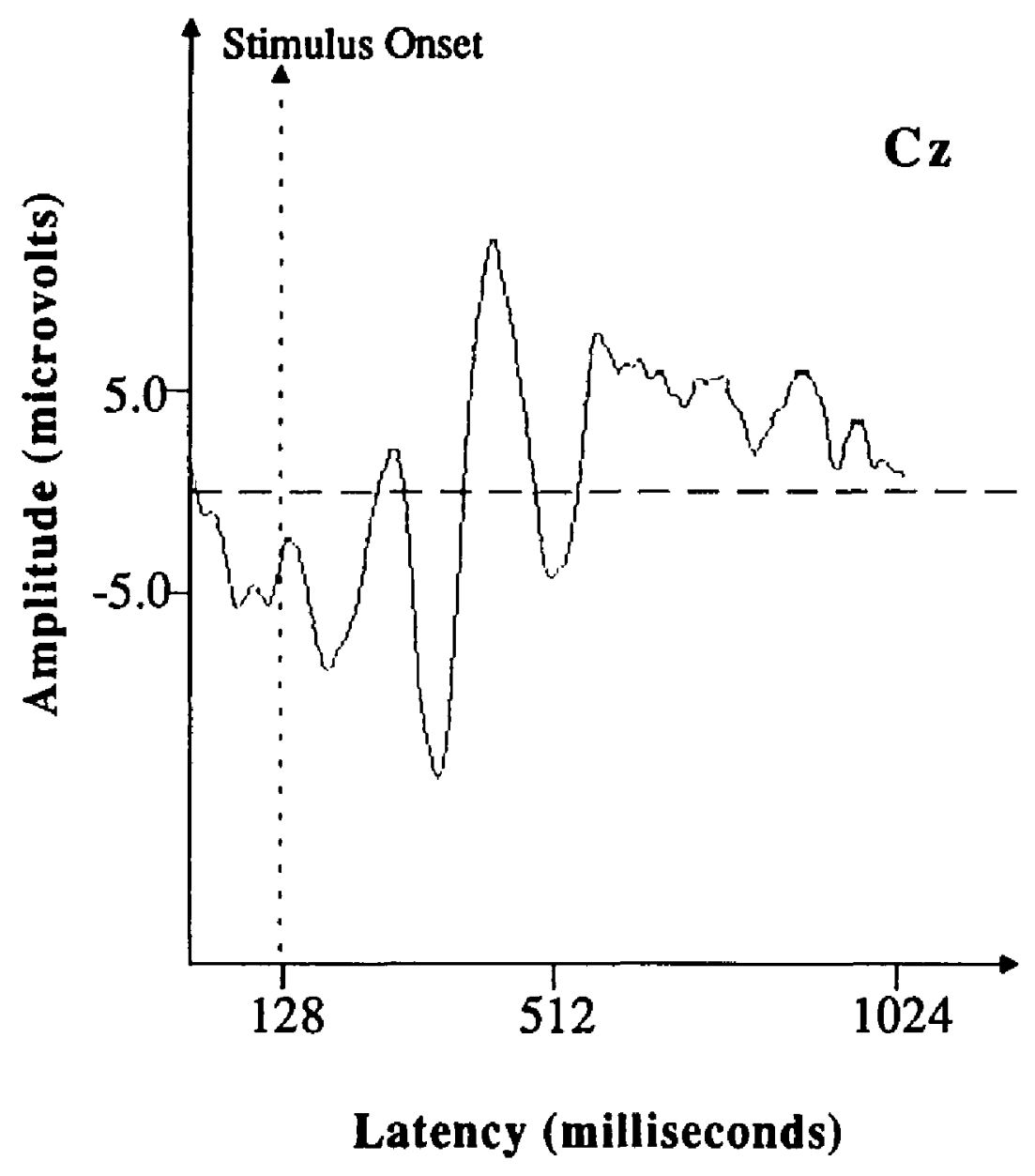

FIGURE 13. Sample waveform from Subject 4. This waveform represents only the responses to plain [ga] in Condition 2 (plain [ga] vs. duplex [ga]). 


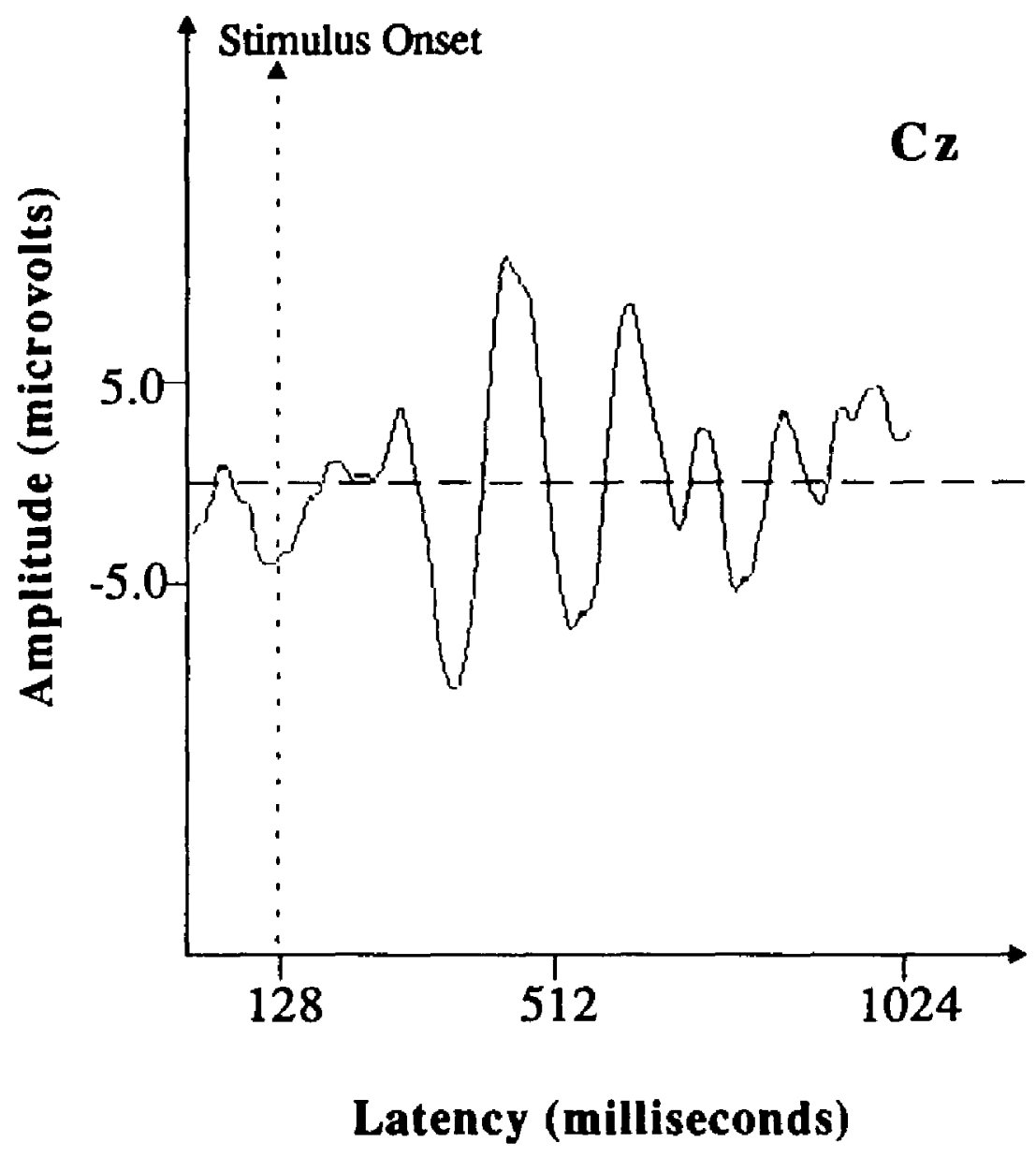

FIGURE 14. Sample waveform from Subject 4. This waveform represents only the responses to duplex [ga] in Condition 2 (plain [ga] vs. duplex [ga]). 


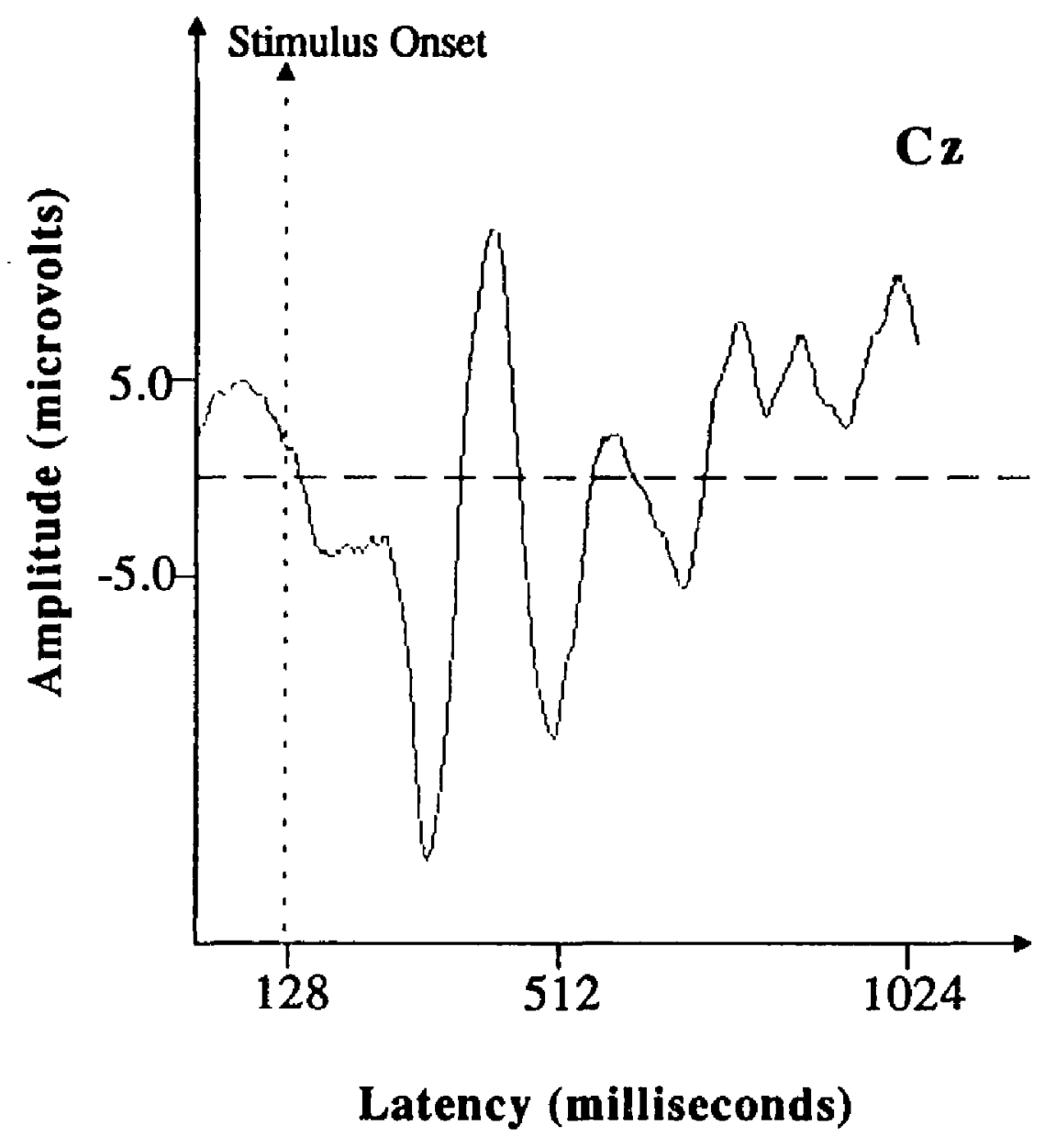

FIGURE 15. Sample waveform from Subject 4. This waveform represents only the responses to duplex [da] in Condition 3 (duplex [da] vs. duplex [ga]). 


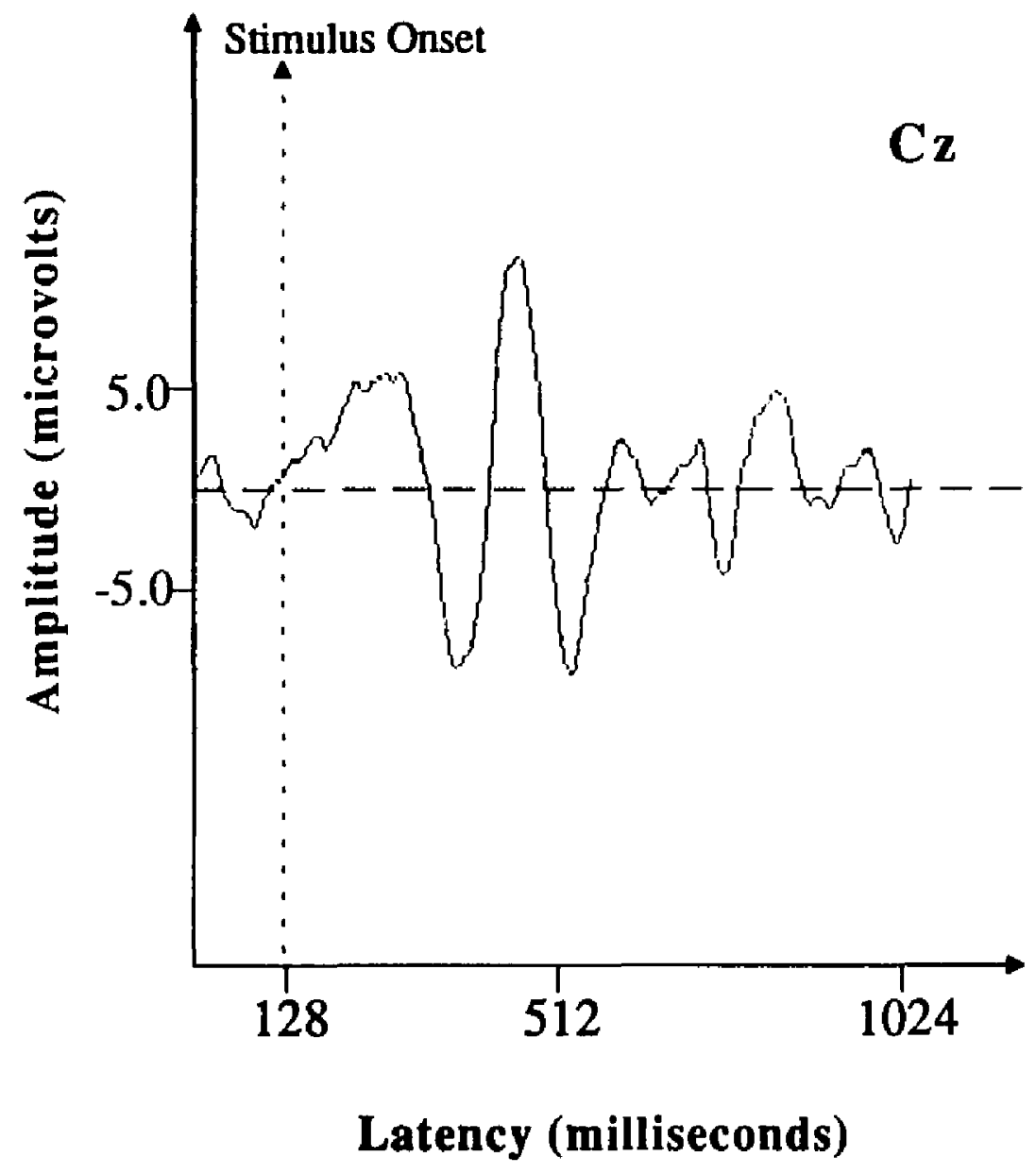

FIGURE 16. Sample waveform from Subject 4. This waveform represents only the responses to duplex [ga] in Condition 3 (duplex [da] vs. duplex [ga]). 


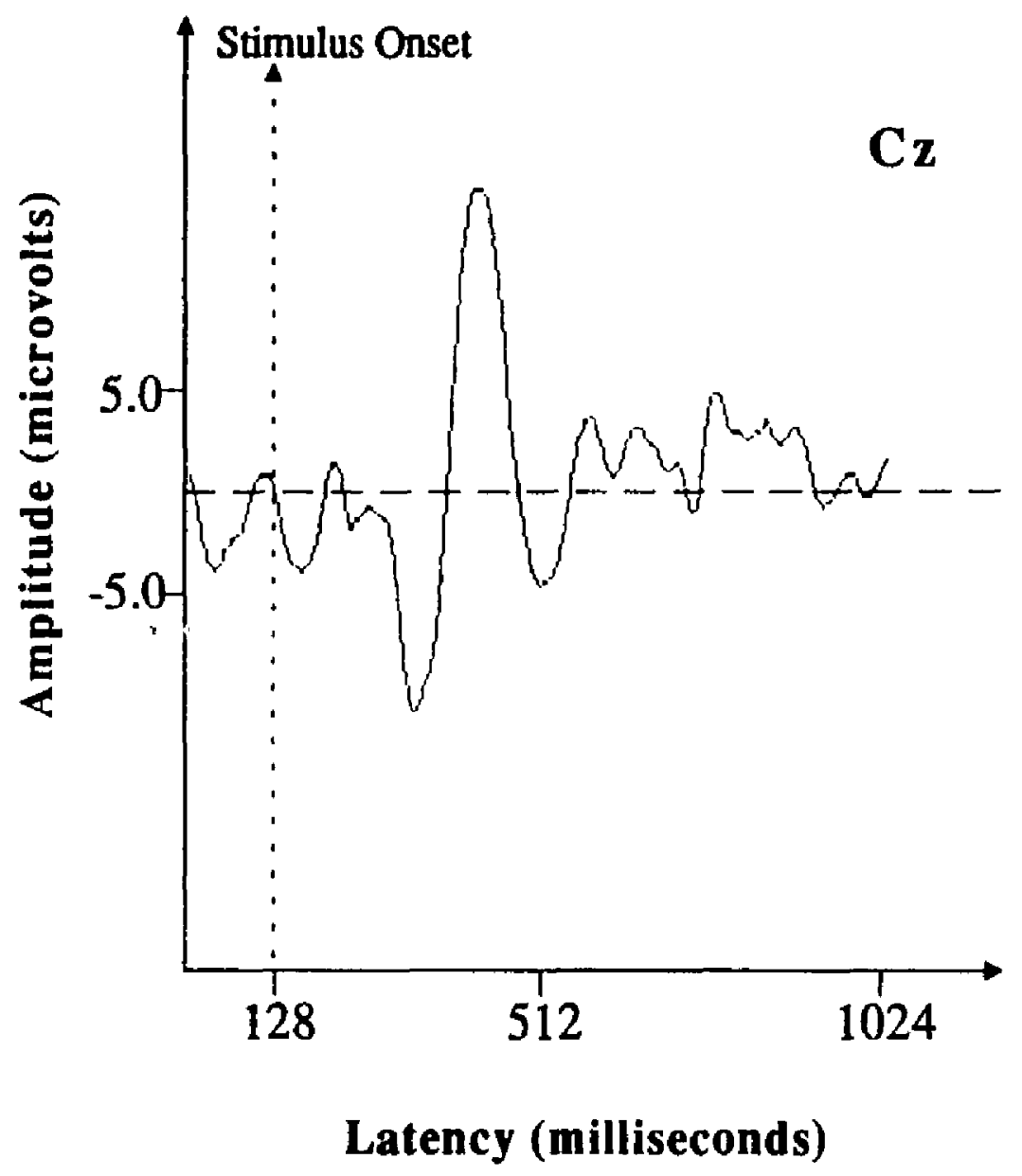

FIGURE 17. Sample waveform from Subject 4. This waveform represents only the responses to plain [da] in Condition 4 (plain [da] vs. plain [ga]). 


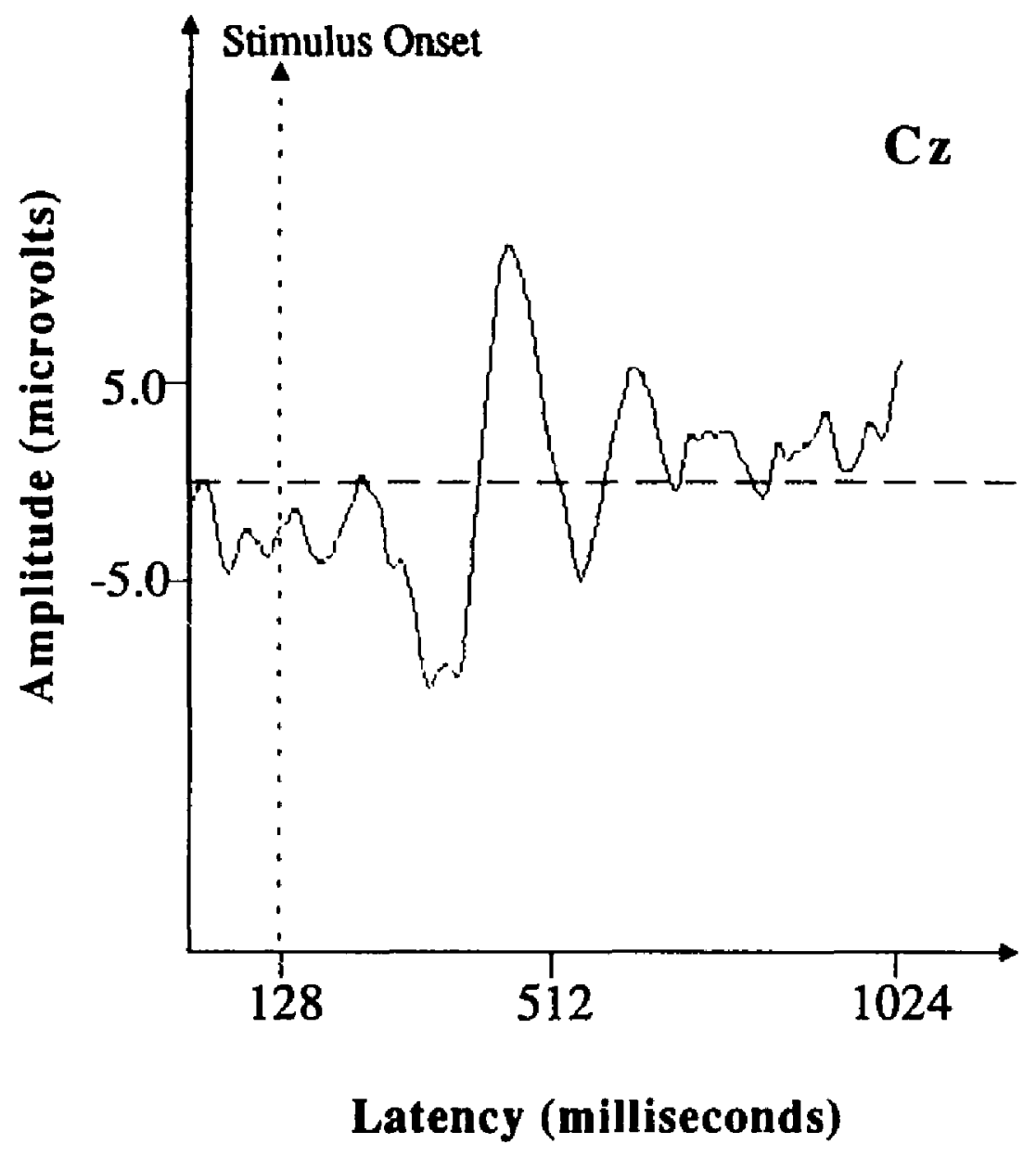

FIGURE 18. Sample waveform from Subject 4. This waveform represents only the responses to plain [ga] in Condition 4 (plain [da] vs. plain [ga]). 


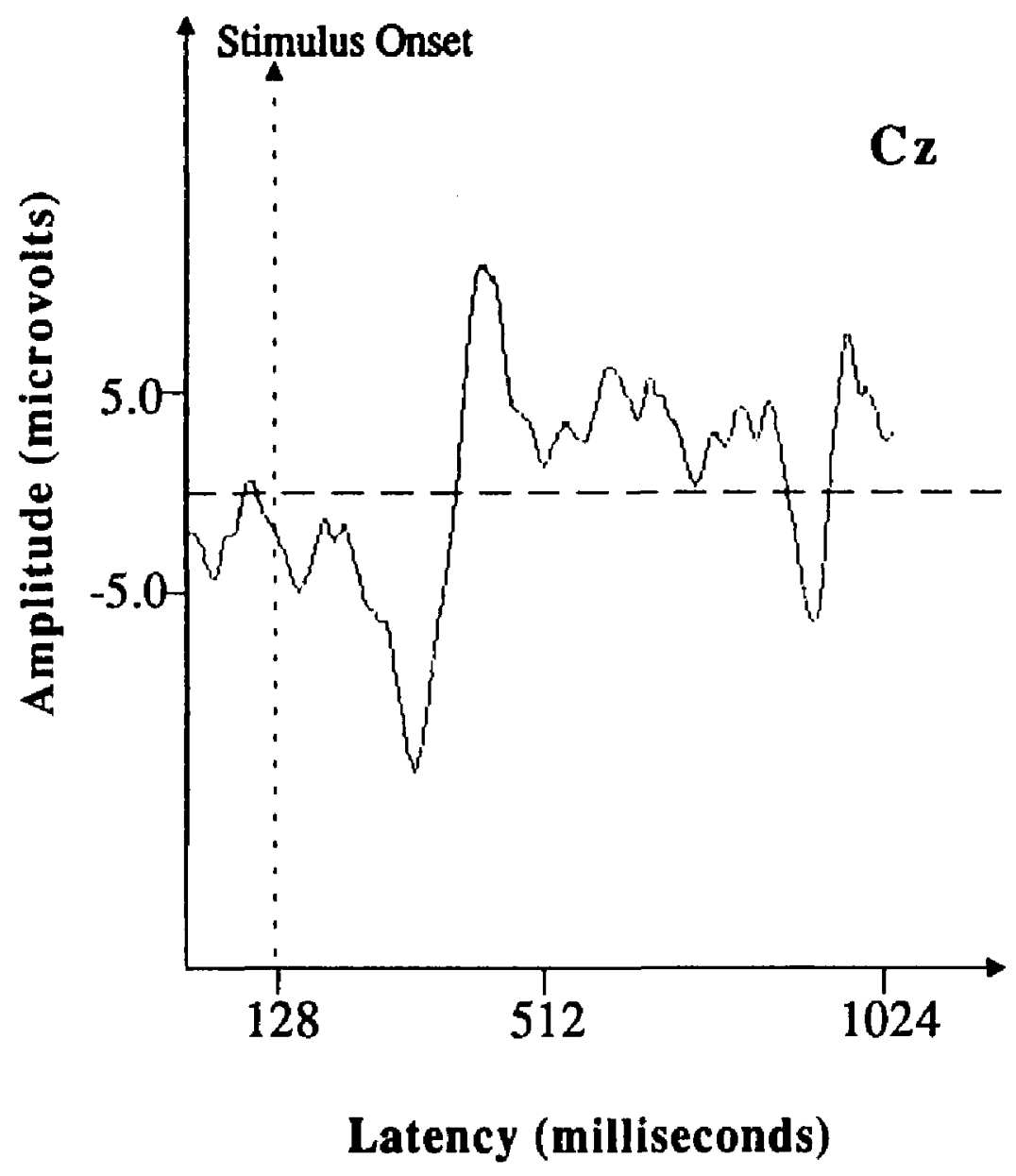

FIGURE 19. Sample waveform from Subject 4. This waveform represents only the responses to tone glide [da] in Condition 5 (tone glide [da] vs. tone glide [ga]). 


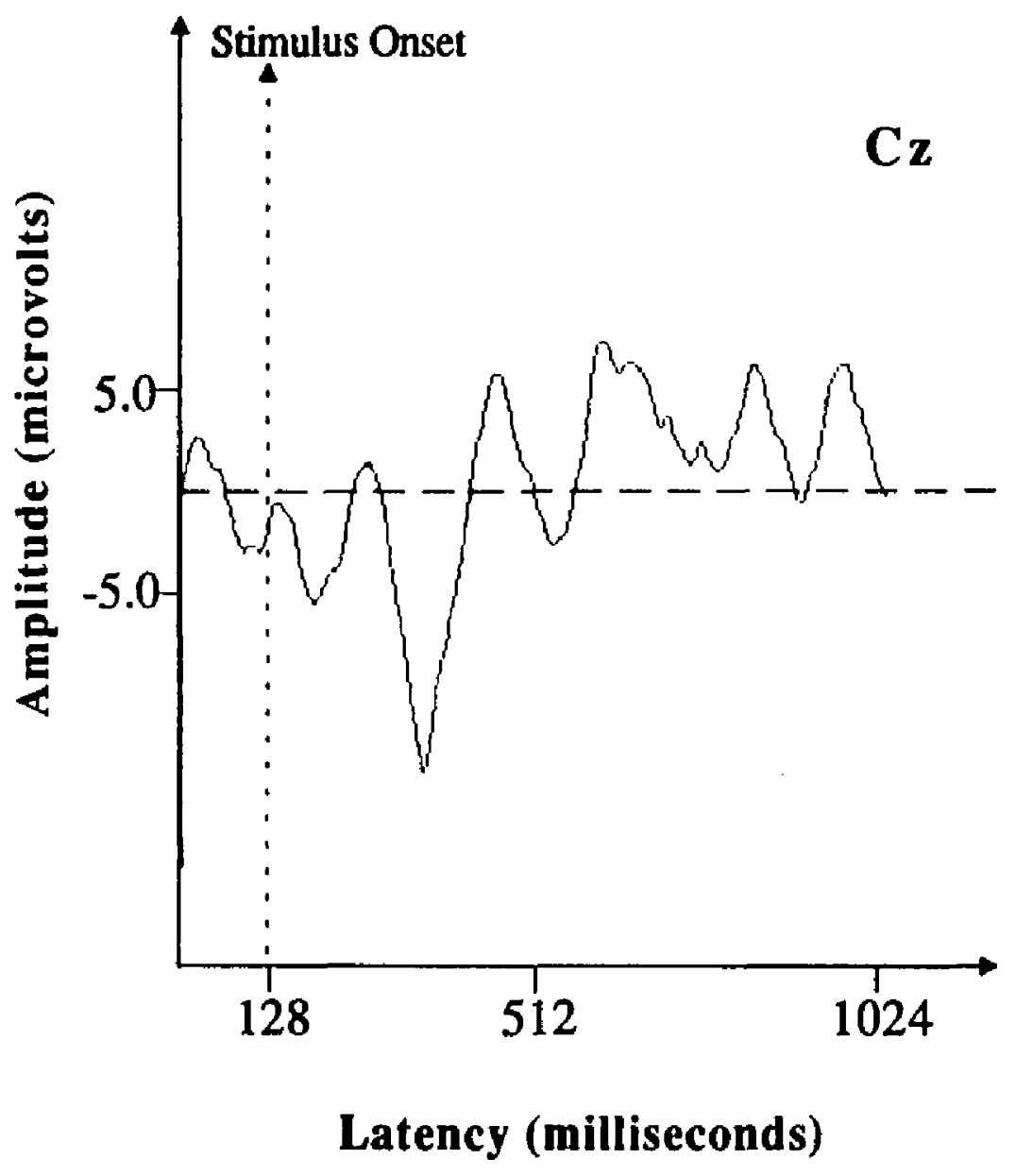

FIGURE 20. Sample waveform from Subject 4. This waveform represents only the responses to tone glide [ga] in Condition 5 (tone glide [da] vs. tone glide [ga]). 


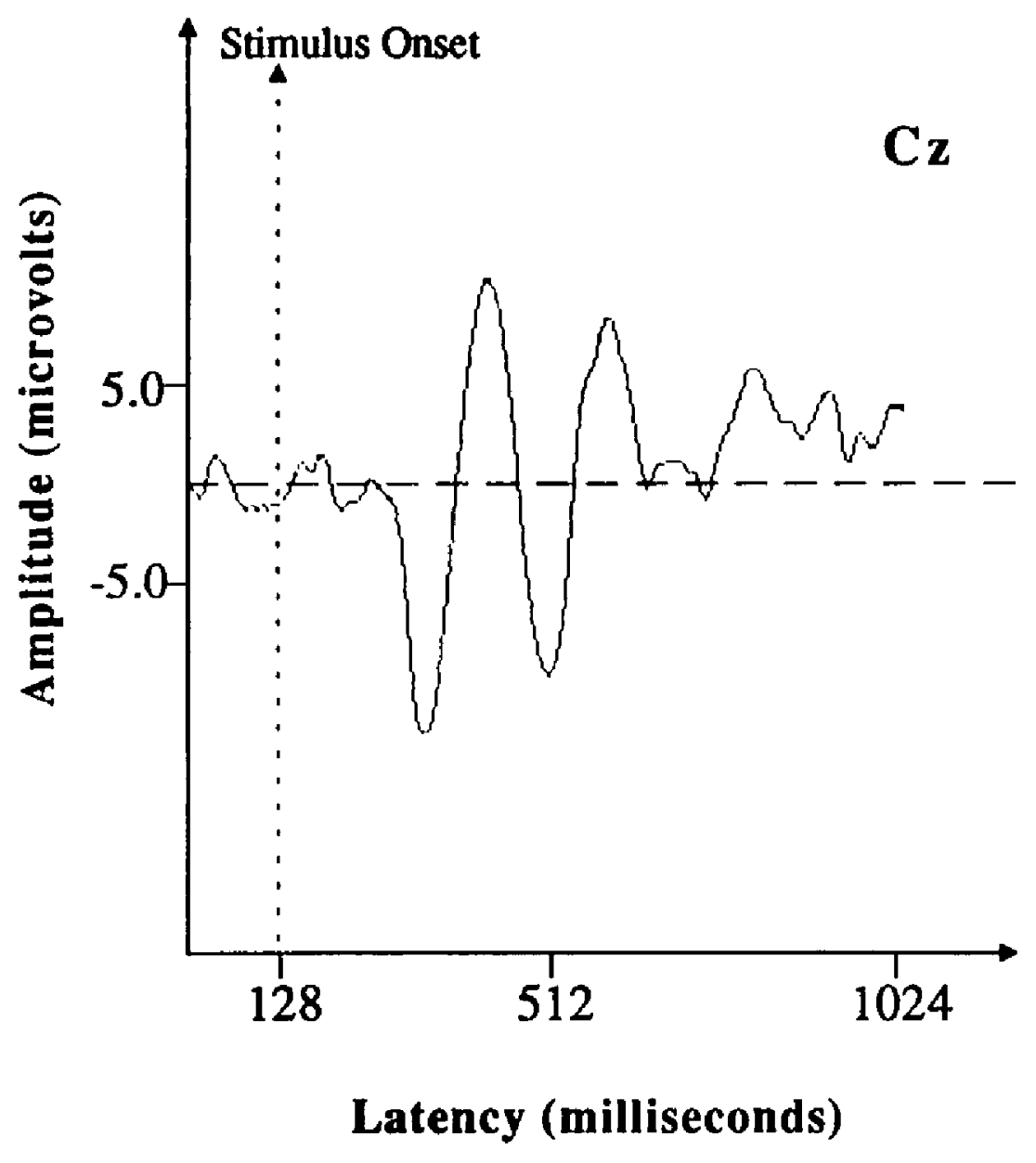

FIGURE 21. Sample waveform from Subject 13. This waveform represents only the response to plain [ga] in Condition 2 (plain [ga] vs. duplex [ga]). 


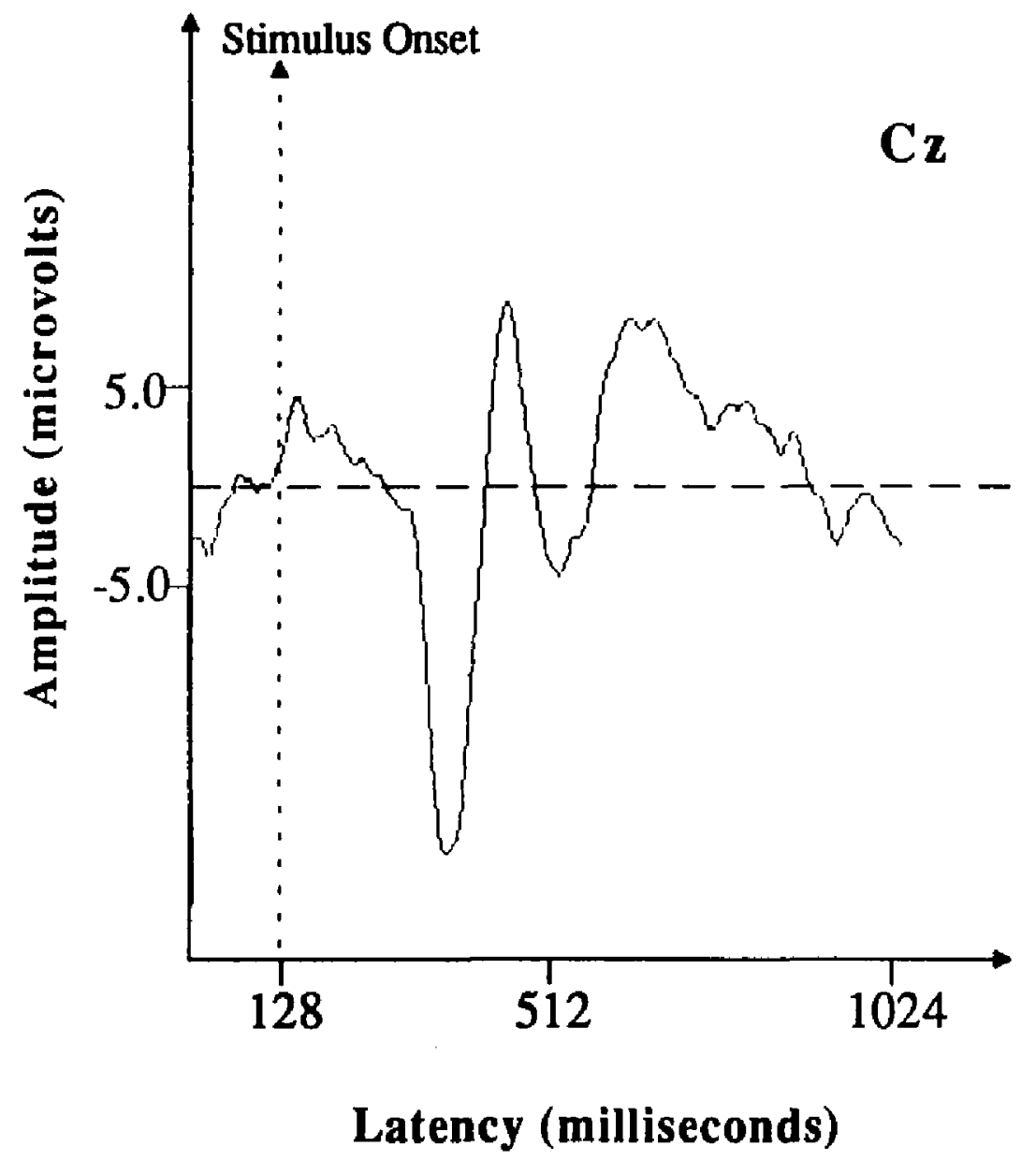

FIGURE 22. Sample waveform from Subject 13 . This waveform represents only the responses to duplex [ga] in Condition 2 (plain [ga] vs. duplex [ga]. 


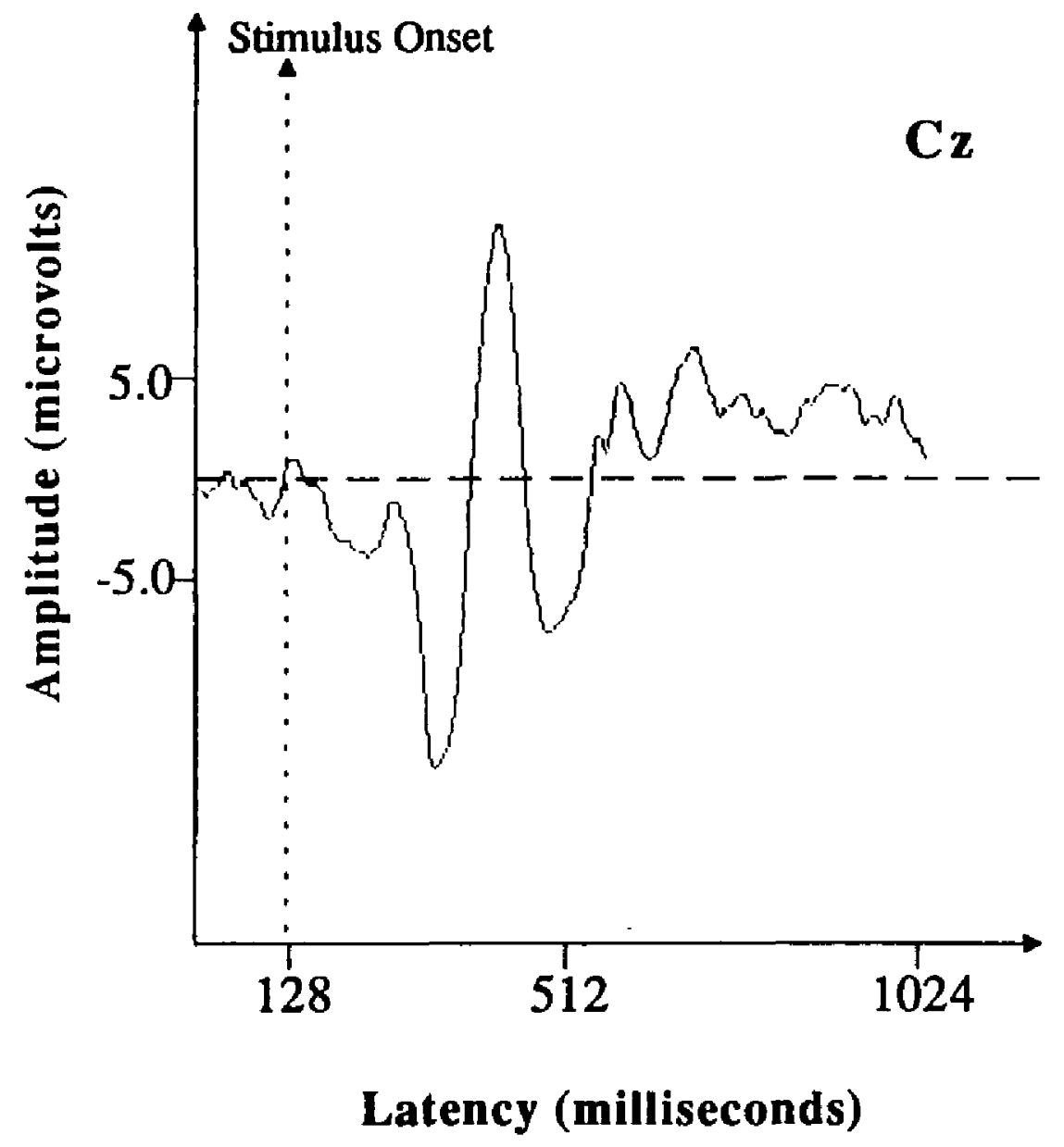

FIGURE 23. Sample waveform from Subject 13. This waveform represents only the responses to duplex [da] in Condition 3 (duplex [da] vs. duplex [ga]. 


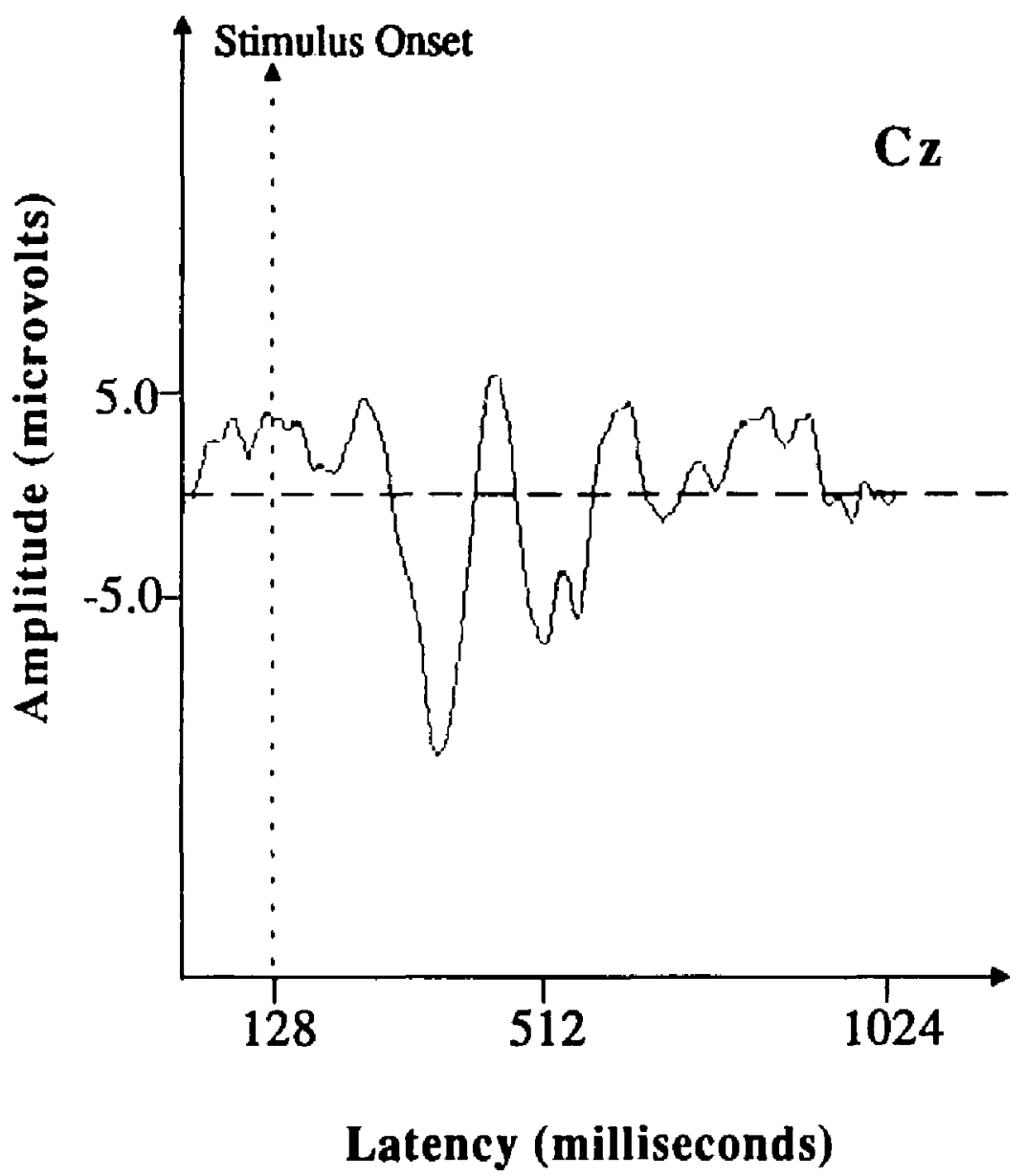

FIGURE 24. Sample waveform from Subjoct 13. This waveform represents only the responses to duplex [ga] in Condition 3 (duplex [da] vs. duplex [ga]. 


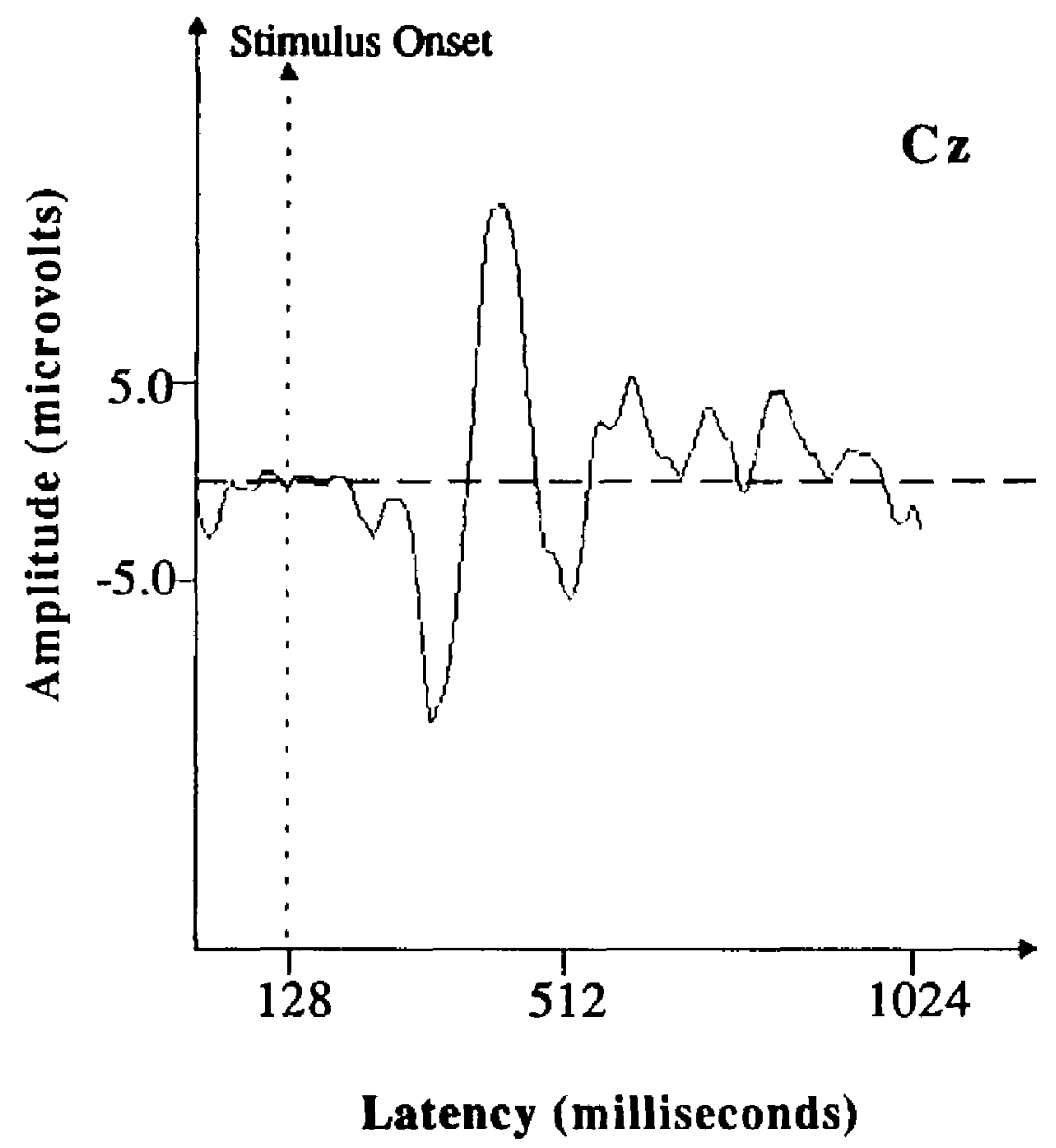

FIGURE 25. Sample waveform from Subject 13. This waveform represents only the responses to plain [da] in Condition 4 (plain [da] vs. plain [ga]). 


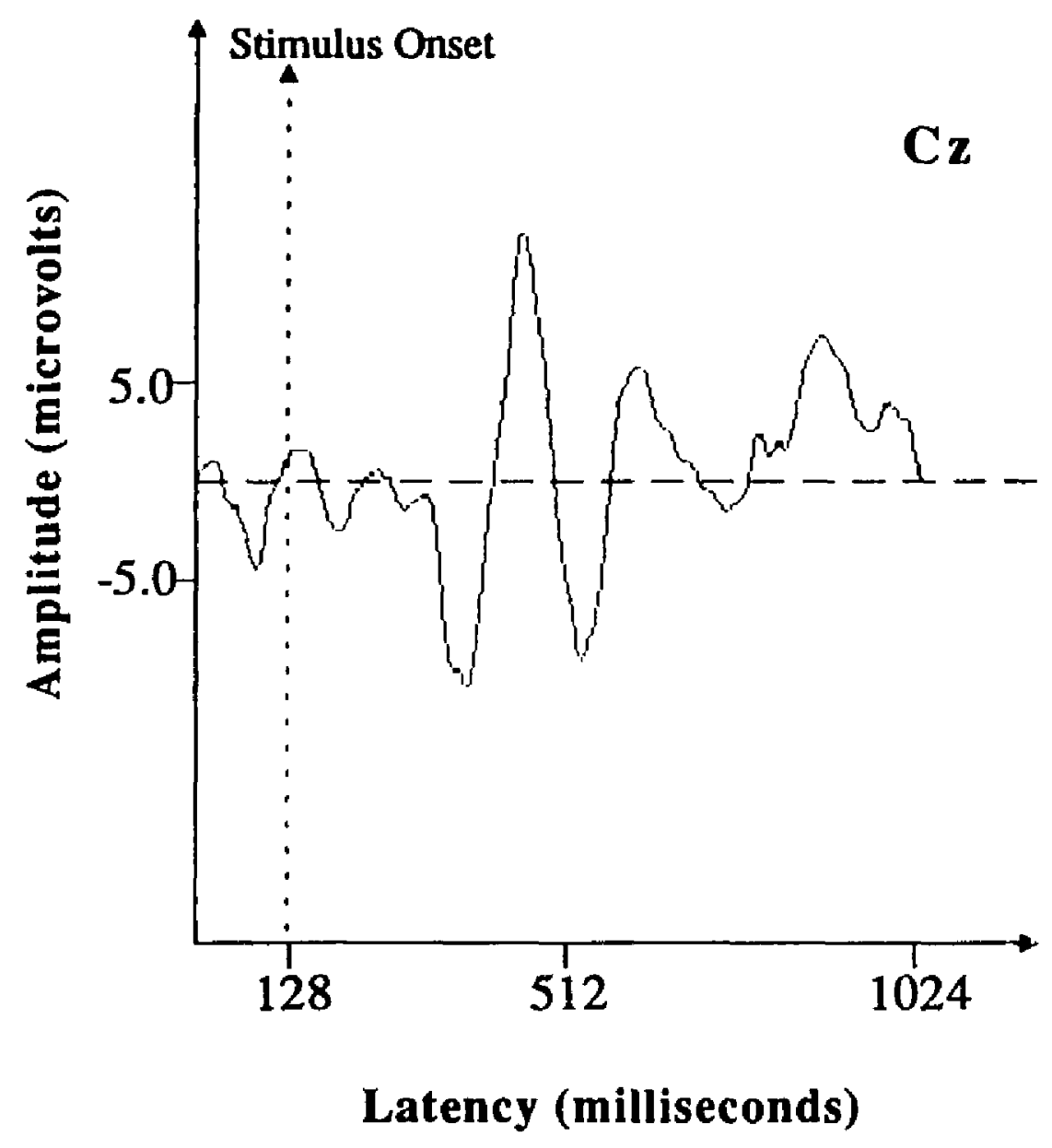

FIGURE 26. Sample waveform from Subject 13. This waveform represents only the responses to plain [ga] in Condition 4 (plain [da] vs. plain [ga]). 


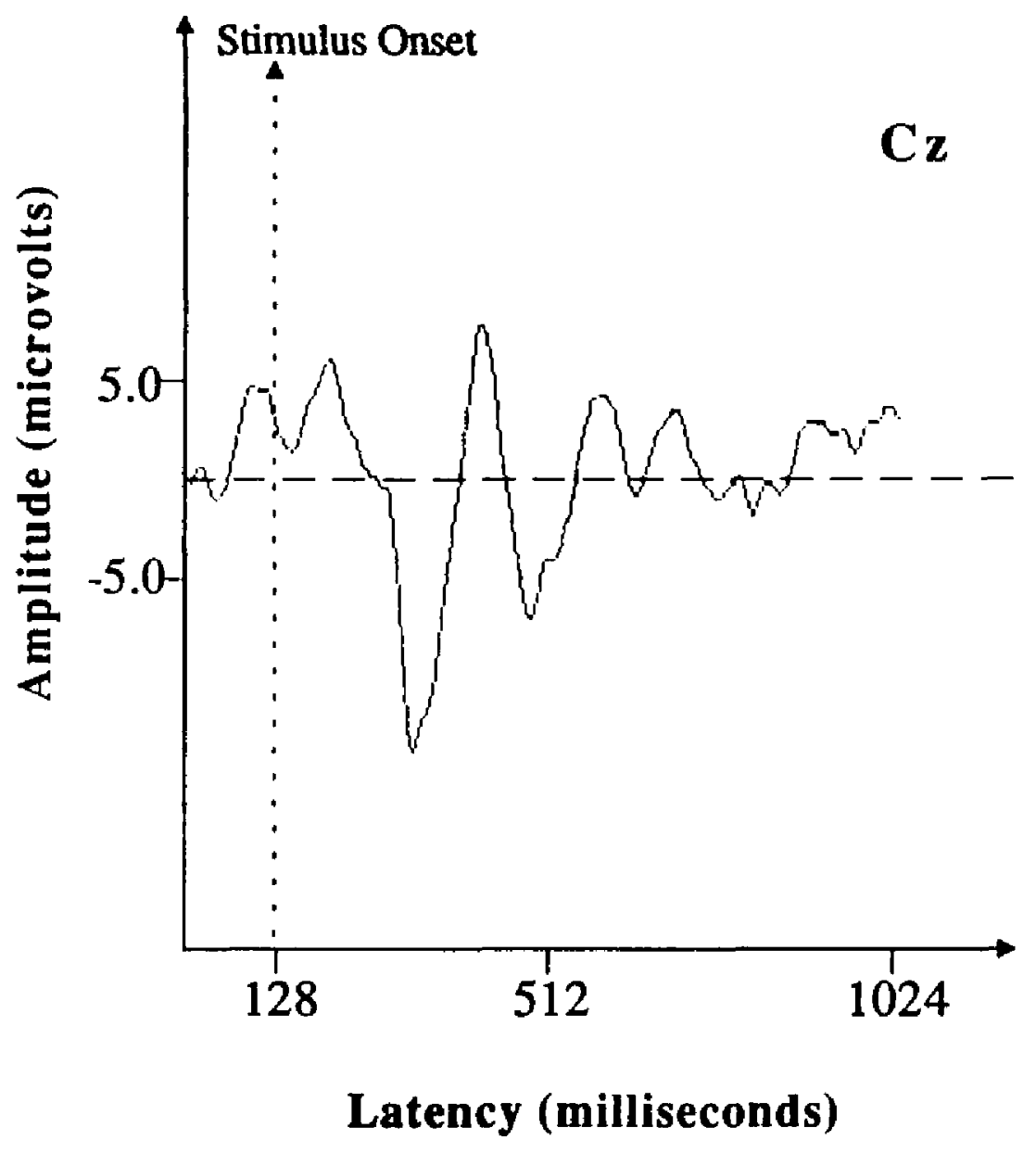

FIGURE 27. Sample waveform from Subjoct 13. This waveform represents only the responses to tone glide [da] in Condition 5 (tone glide [da] vs. tone glide [ga]. 


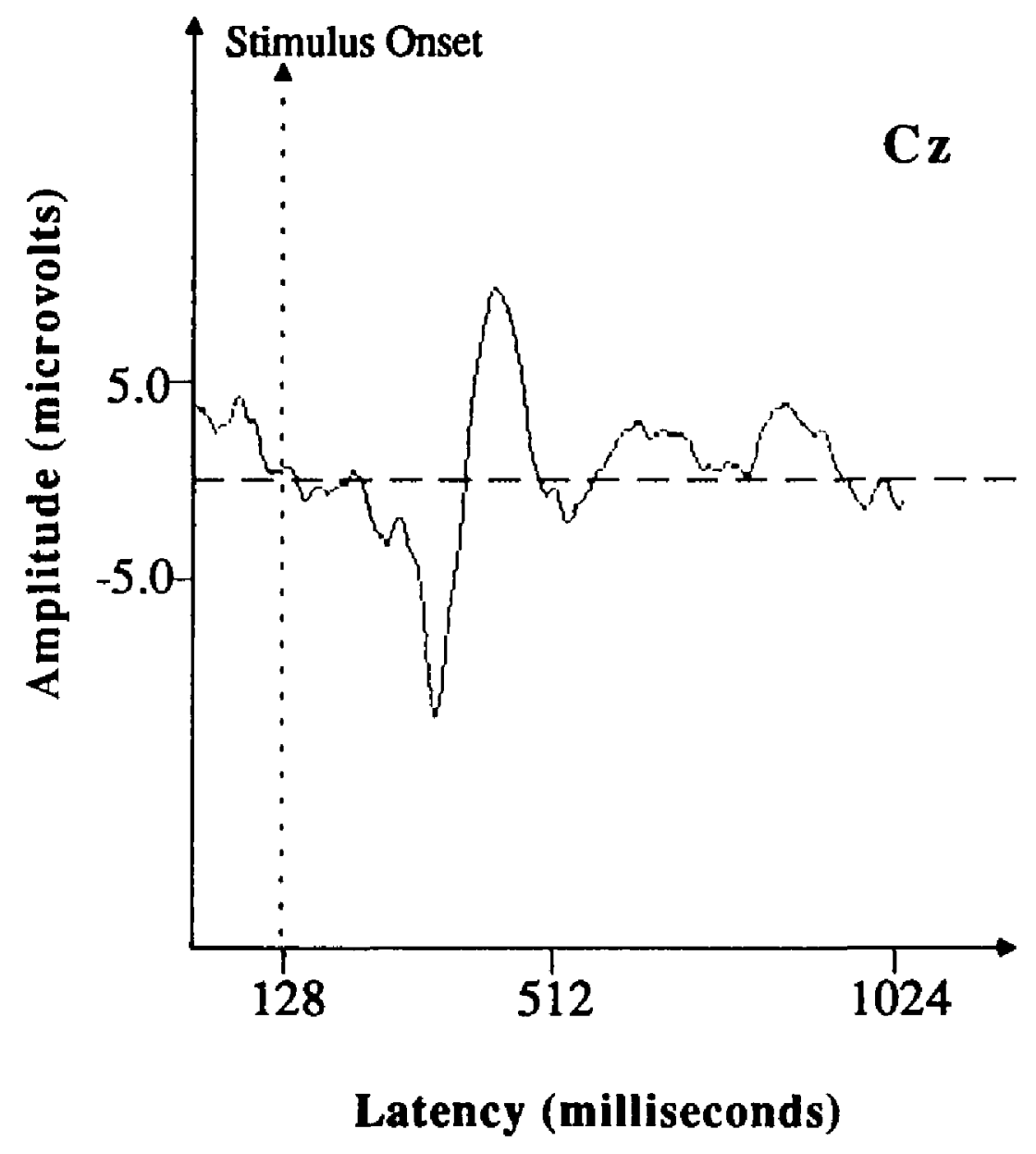

FIGURE 28. Sample waveform from Subject 13. This waveform represents only the responses to tone glide [ga] in Condition 5 (tone glide [da] vs. tone glide [ga]. 


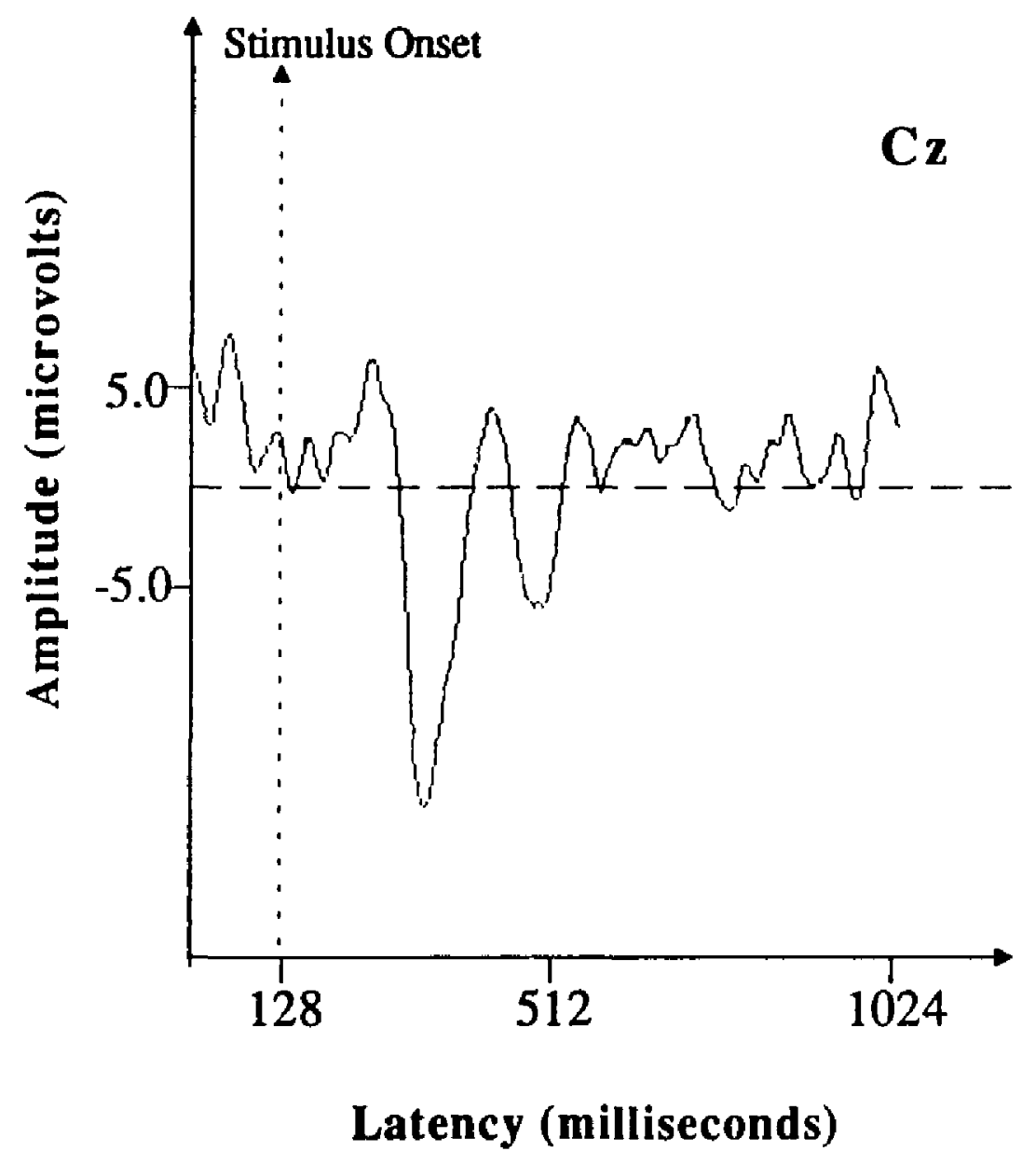

FIGURE 29. Sample waveform from Subject 19. This waveform represents only the responses to plain [da] in Condition 1 (plain [da] vs. duplex [da]). 


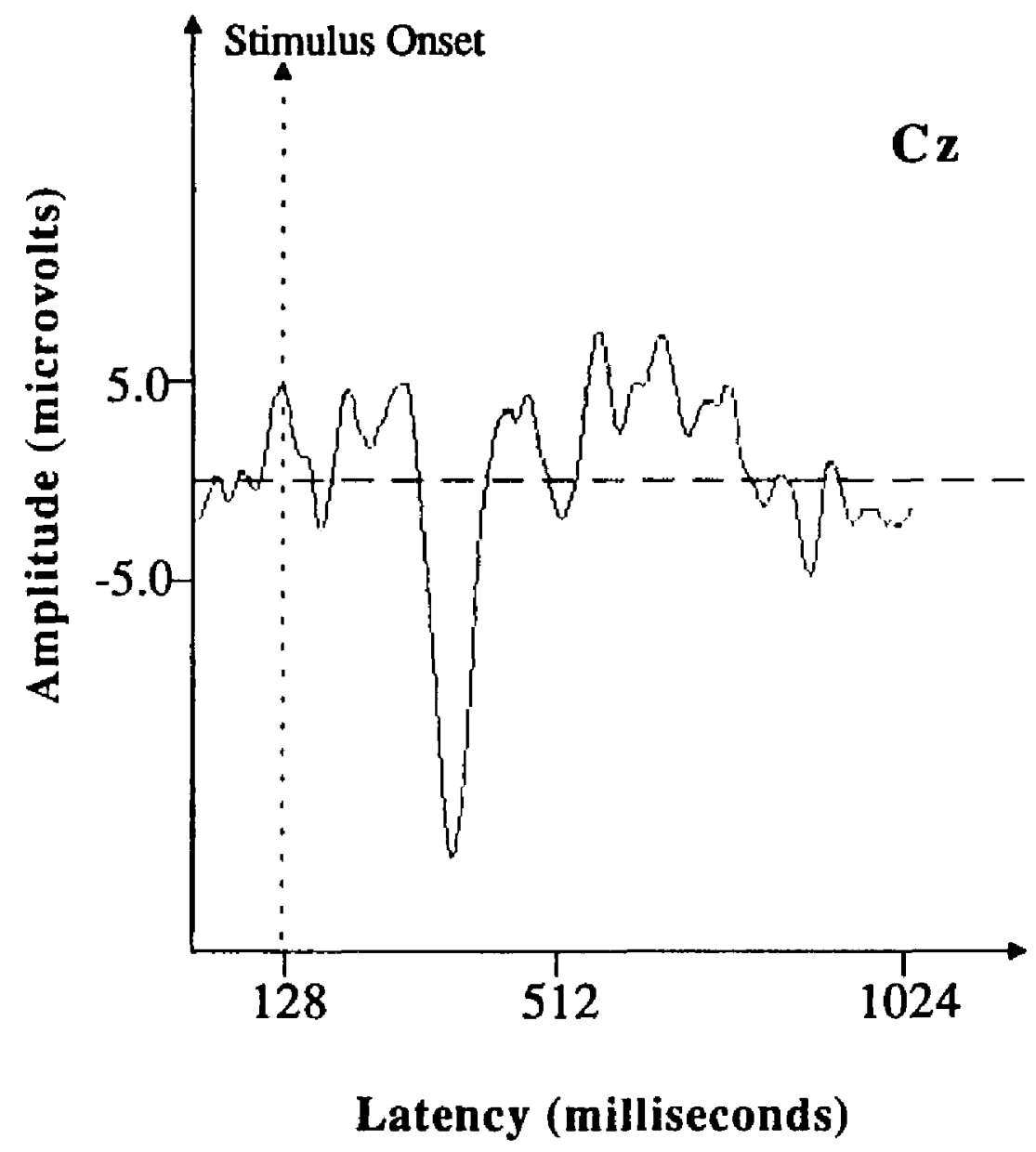

FIGURE 30. Sample waveform from Subject 19. This waveform represents only the responses to duplex [da] in Condition 1 (plain [da] vs. duplex [da]). 


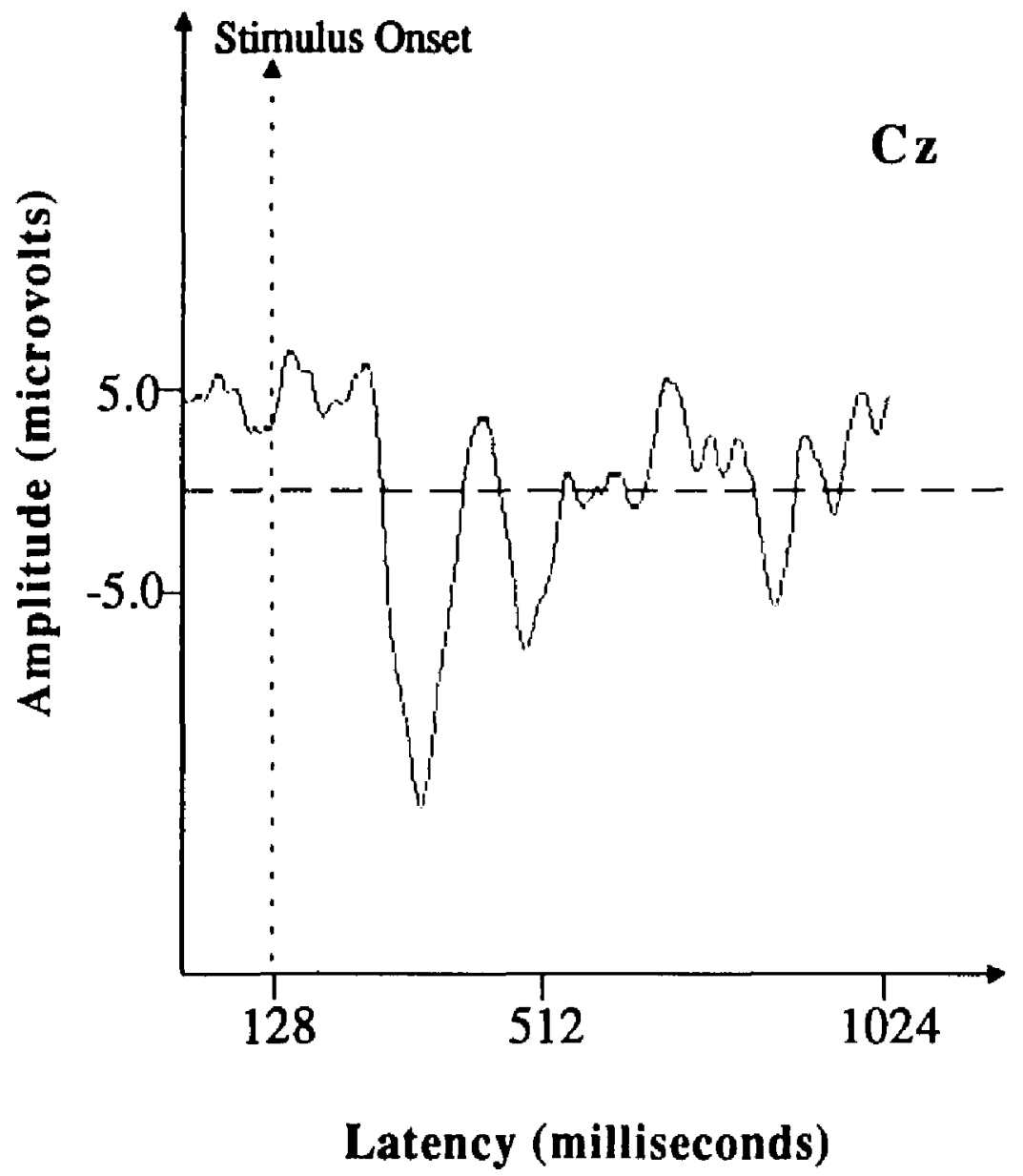

FIGURE 31. Sample waveform from Subject 19. This waveform represents only the responses to plain [ga] in Condition 2 (plain [ga] vs. duplex [ga]). 


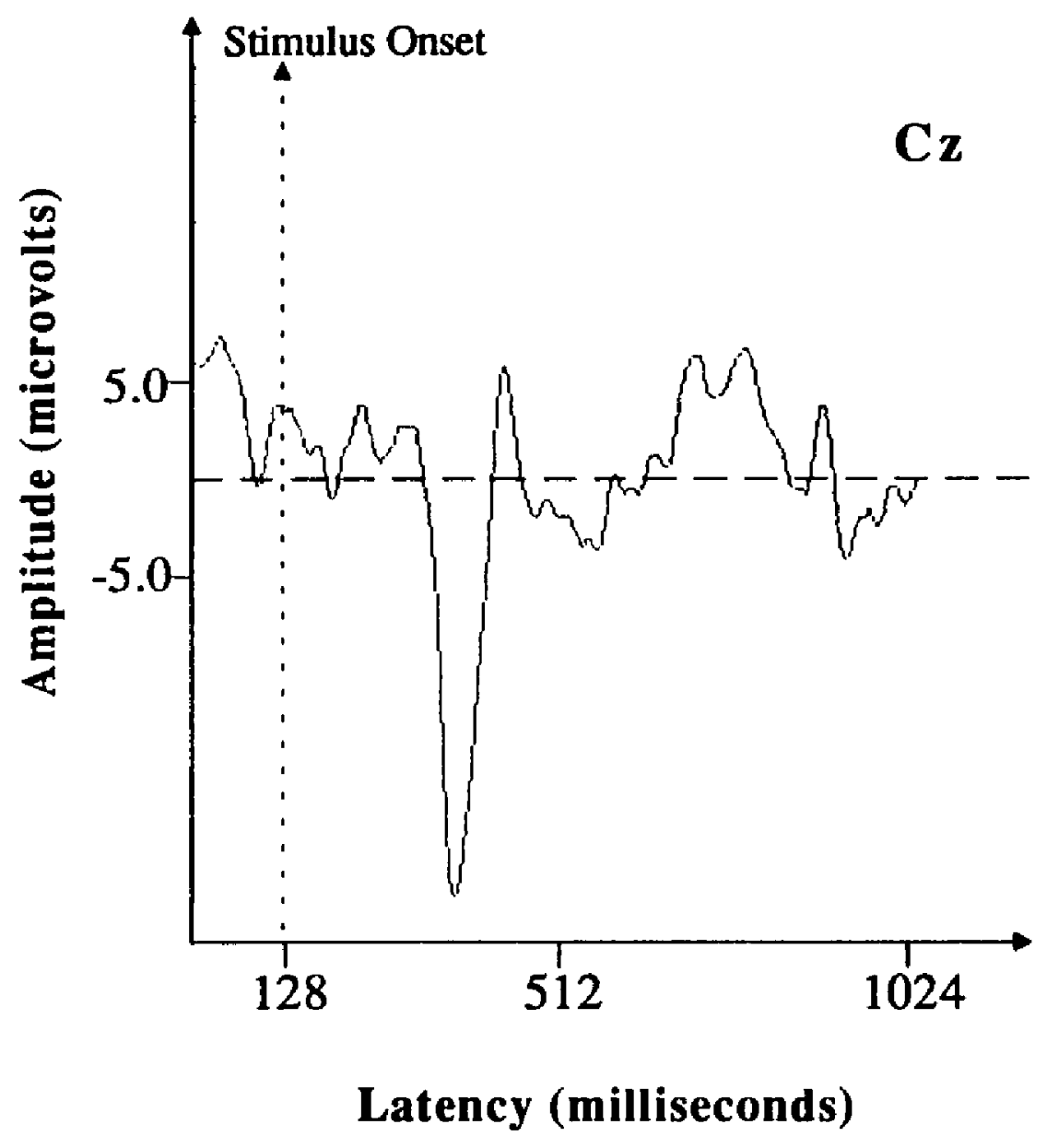

FIGURE 32. Sample waveform from Subject 19. This waveform represents only the responses to duplex [ga] in Condition 2 (plain [ga] vs. duplex [ga]). 


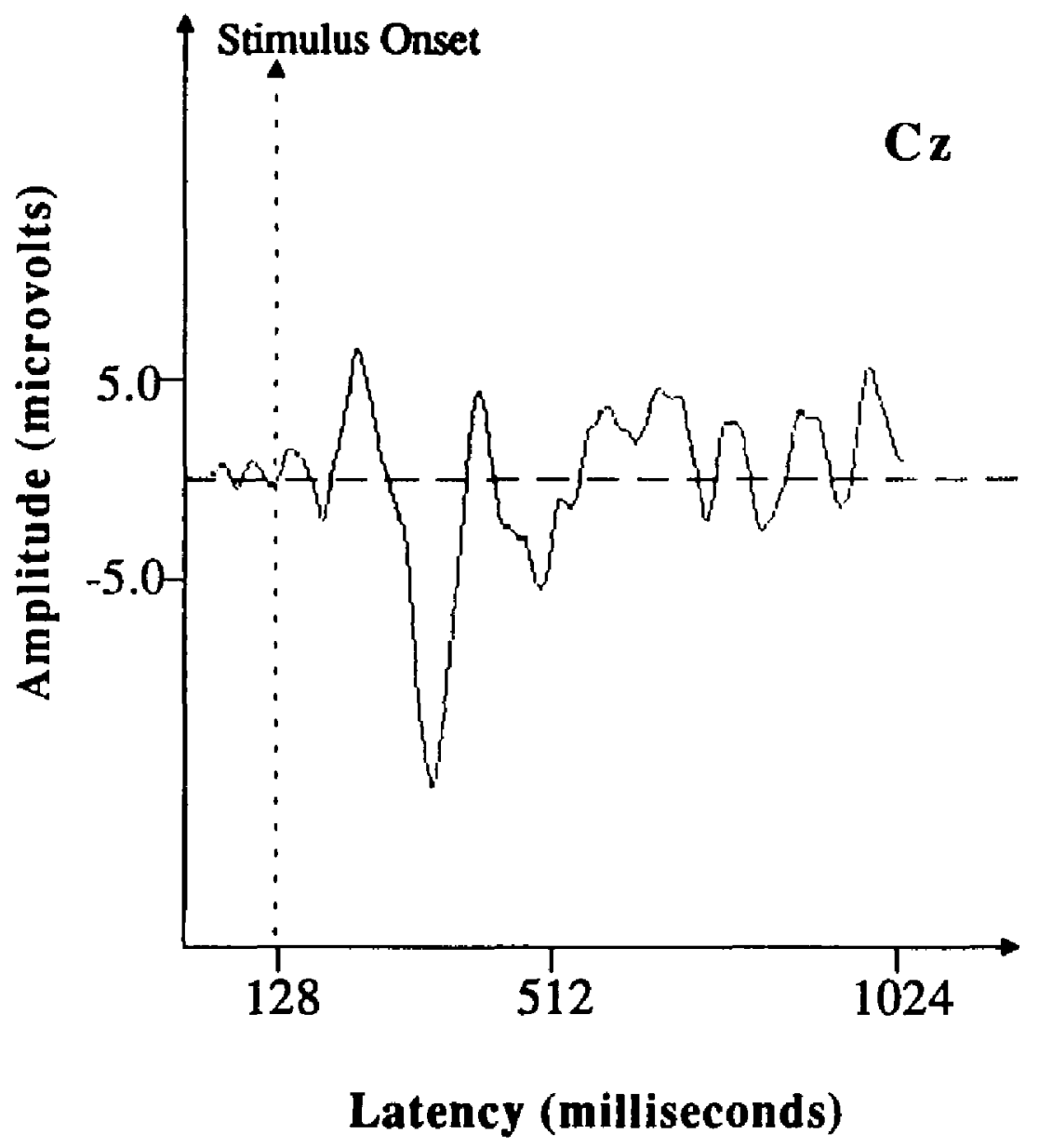

FIGURE 33. Sample waveform from Subject 19. This waveform represents only the responses to duplex [da] in Condition 3 (duplex [da] vs. duplex [ga]). 


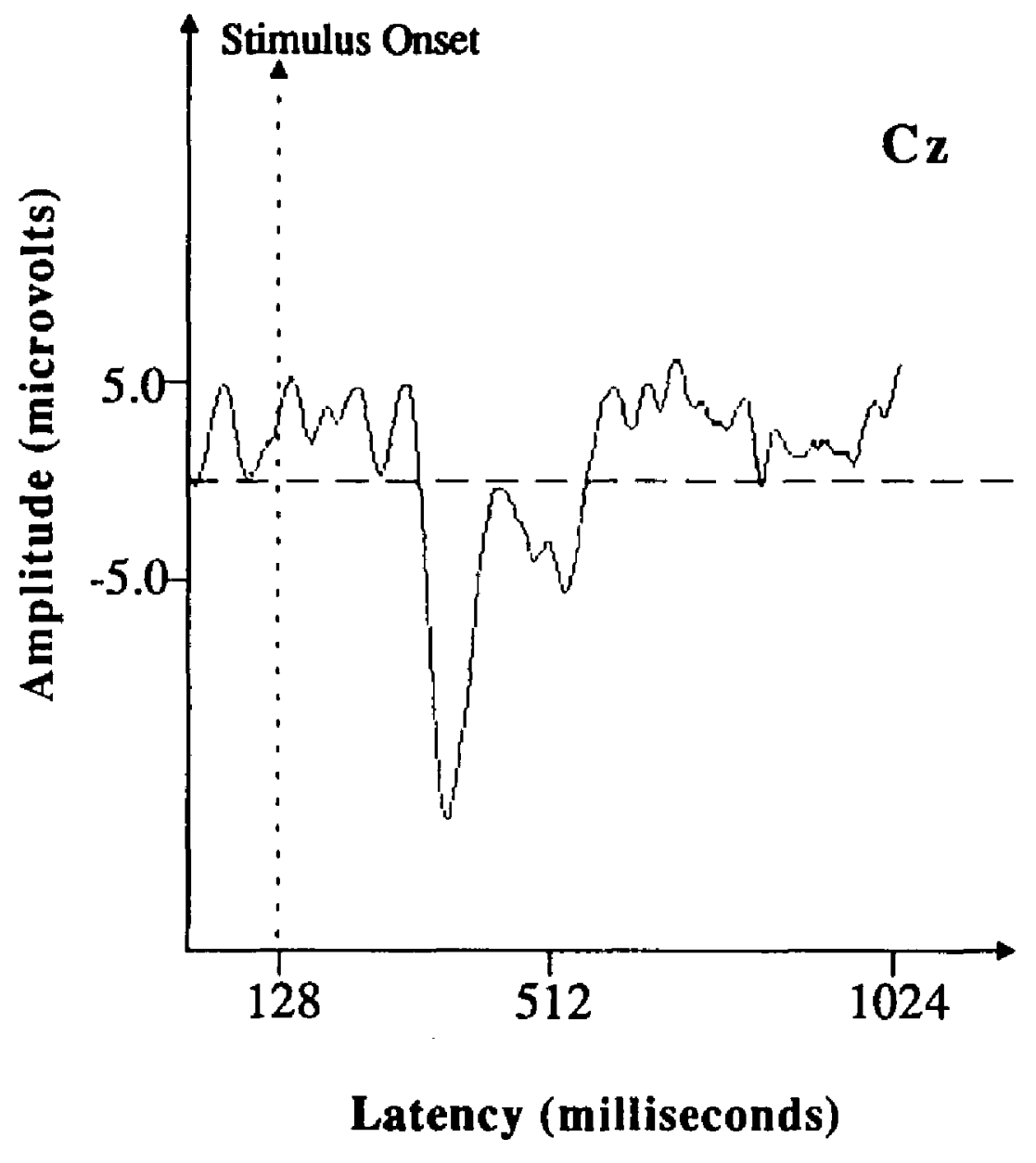

FIGURE 34. Sample waveform from Subject 19. This waveform represents only the responses to duplex [ga] in Condition 3 (duplex [da] vs. duplex [ga]). 


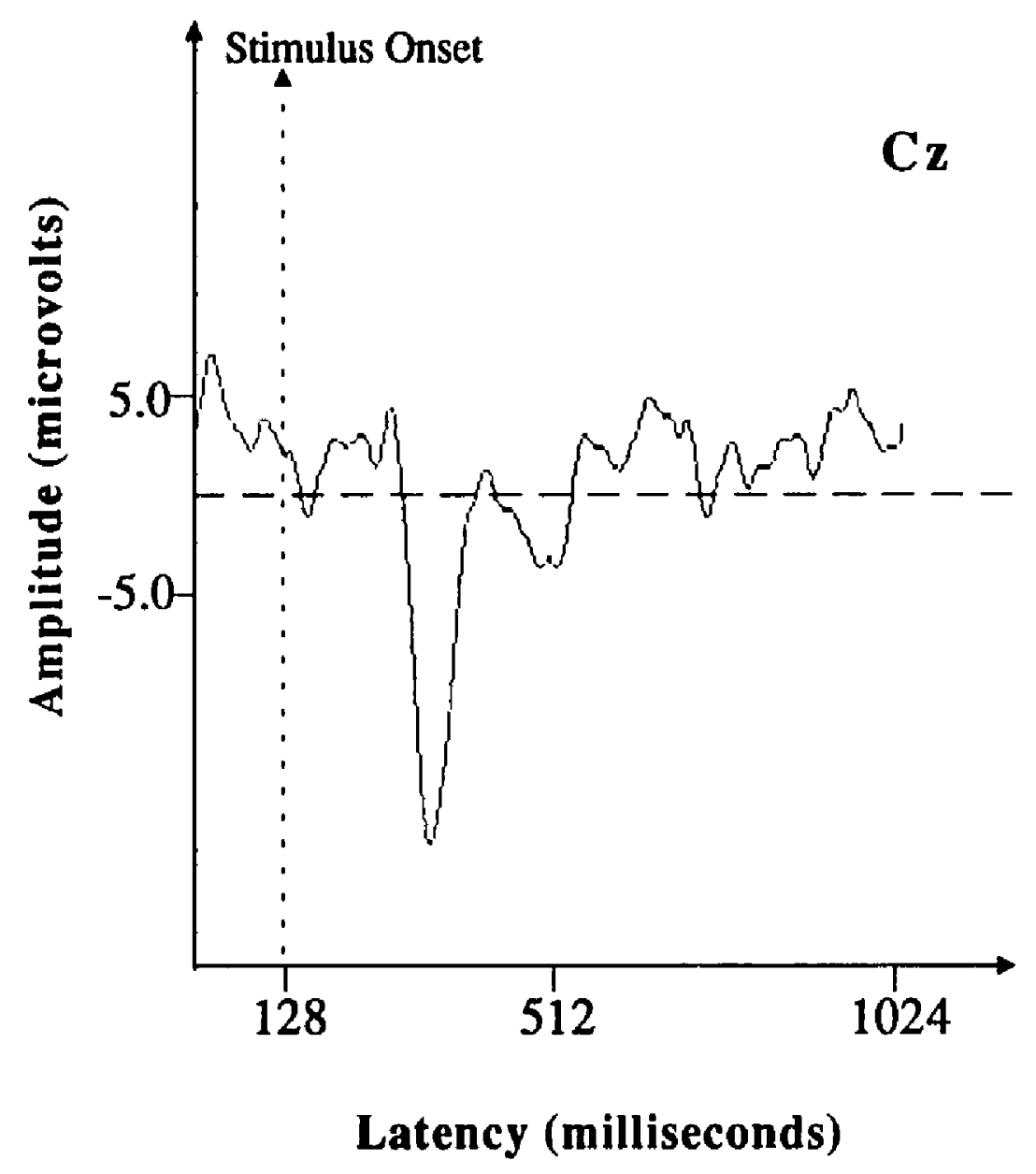

FIGURE 35. Sample waveform from Subject 19. This waveform represents only the responses to plain [da] in Condition 4 (plain [da] vs. plain [ga]). 


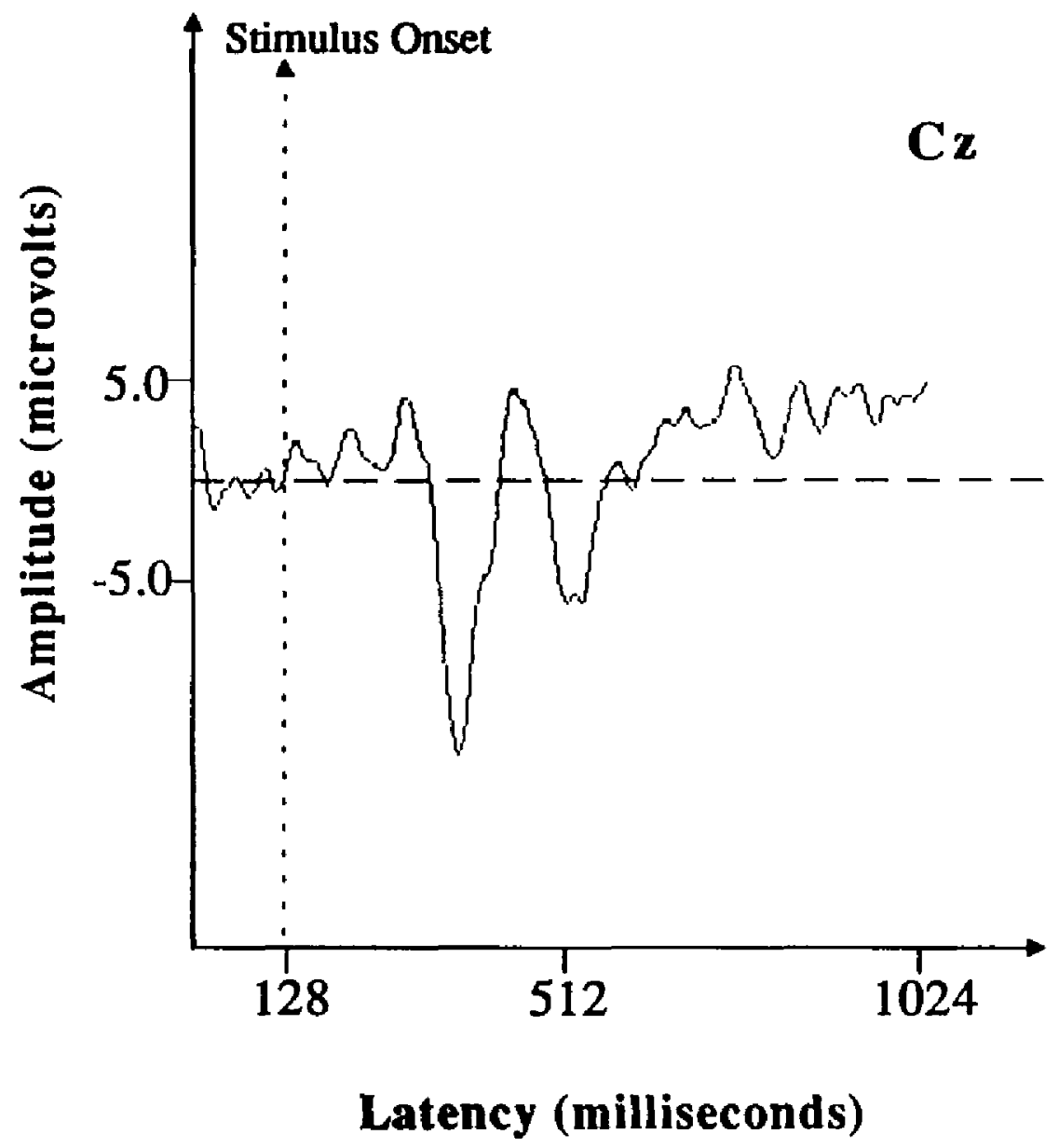

FIGURE 36. Sample waveform from Subject 19. This waveform represents only the responses to plain [ga] in Condition 4 (plain [da] vs. plain [ga]). 


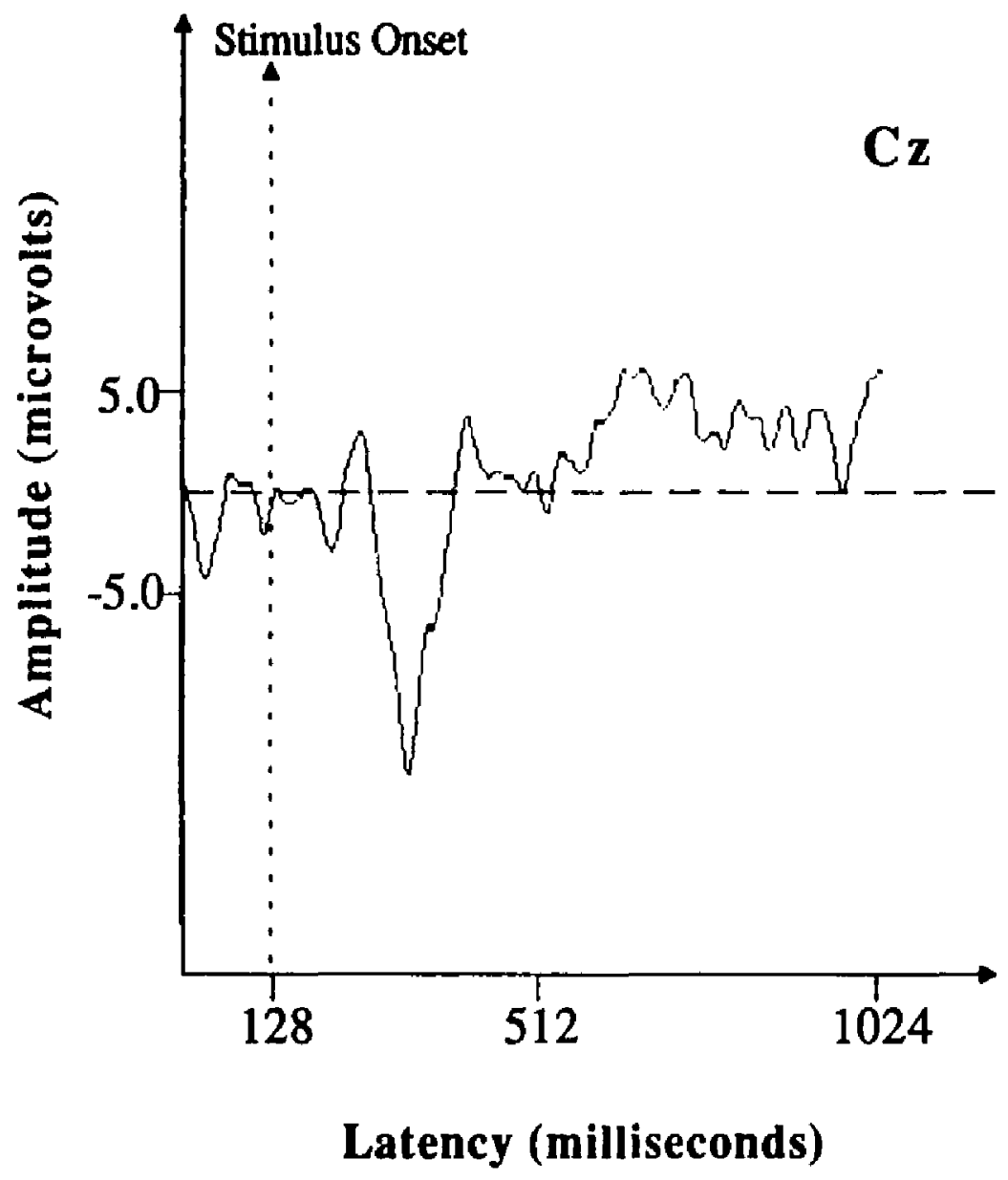

FIGURE 37. Sample waveform from Subject 19. This waveform represents only the responses to tone glide [da] in Condition 5 (tone glide [da] vs. tone glide [ga]). 


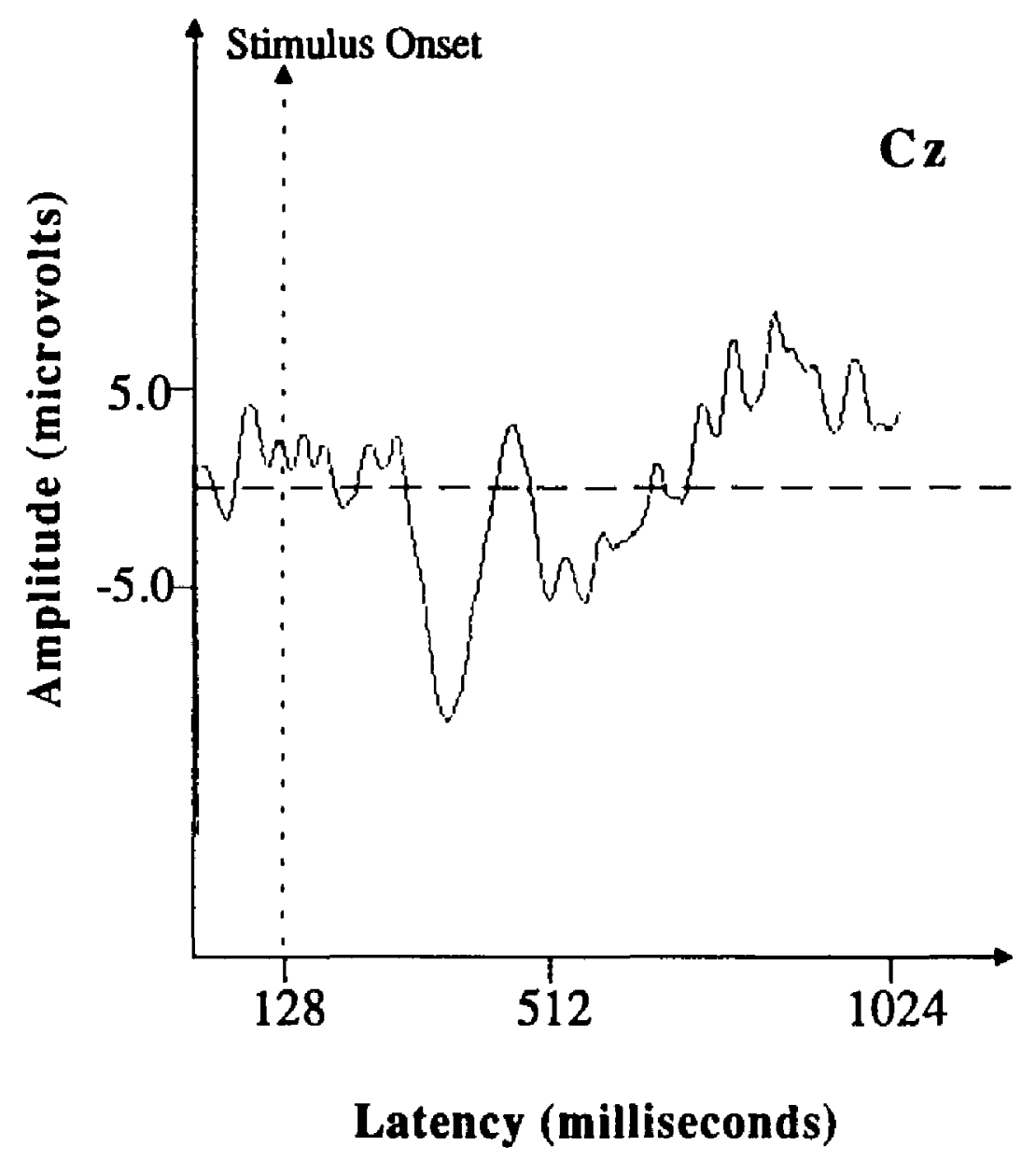

FIGURE 38. Sample waveform from Subject 19. This waveform represents only the responses to tone glide [ga] in Condition 5 (tone glide [da] vs. tone glide [ga]). 
APPENDIX C

GRAND AVERAGE ERP WAVEFORMS FOR EACH STIMULUS WITHIN EACH CONDITION 


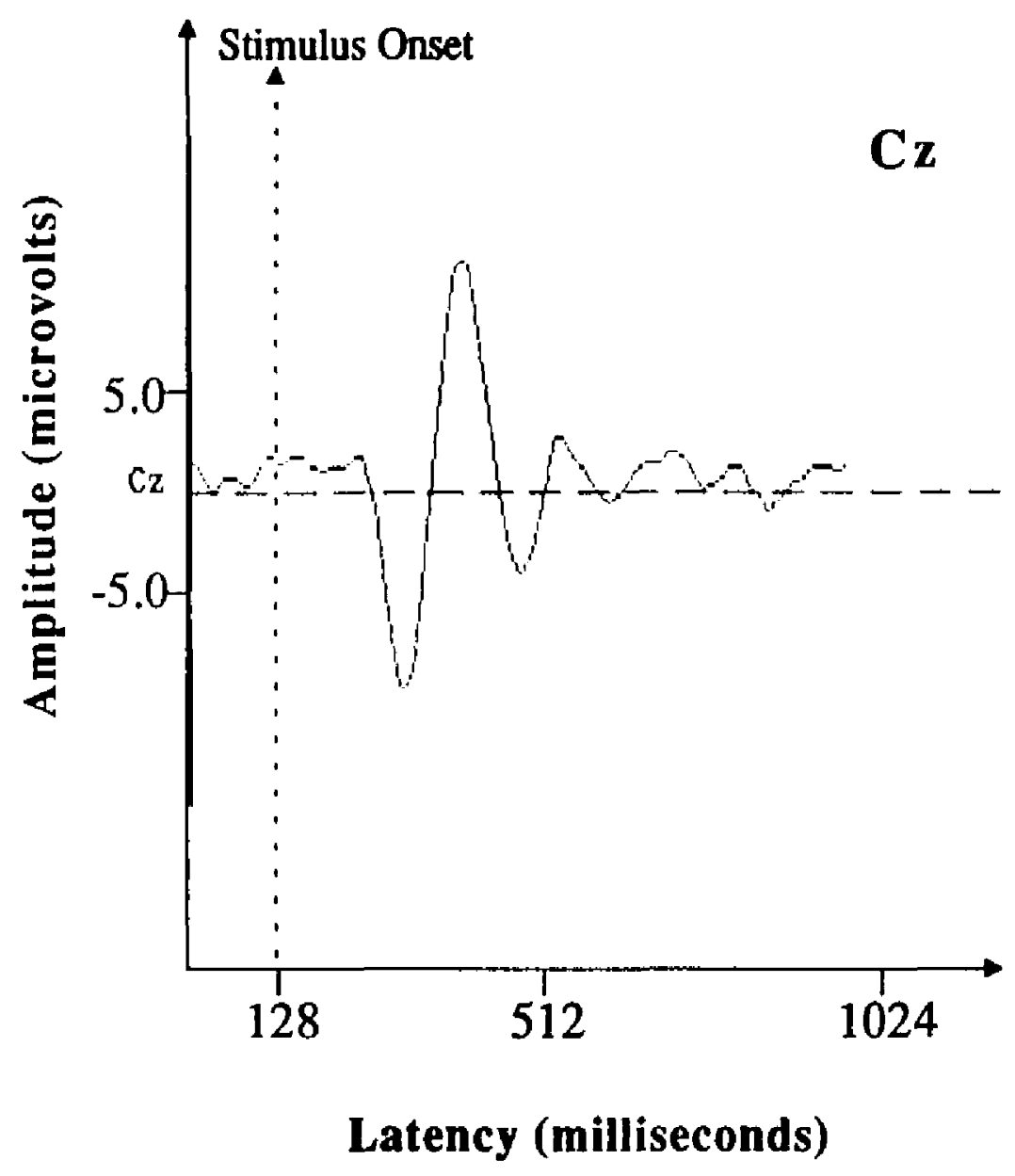

FIGURE 39. Grand average waveform across all subjocts in Condition 1 (plain [da] vs. duplex [da]). This waveform represents only the responses to plain [da]. 


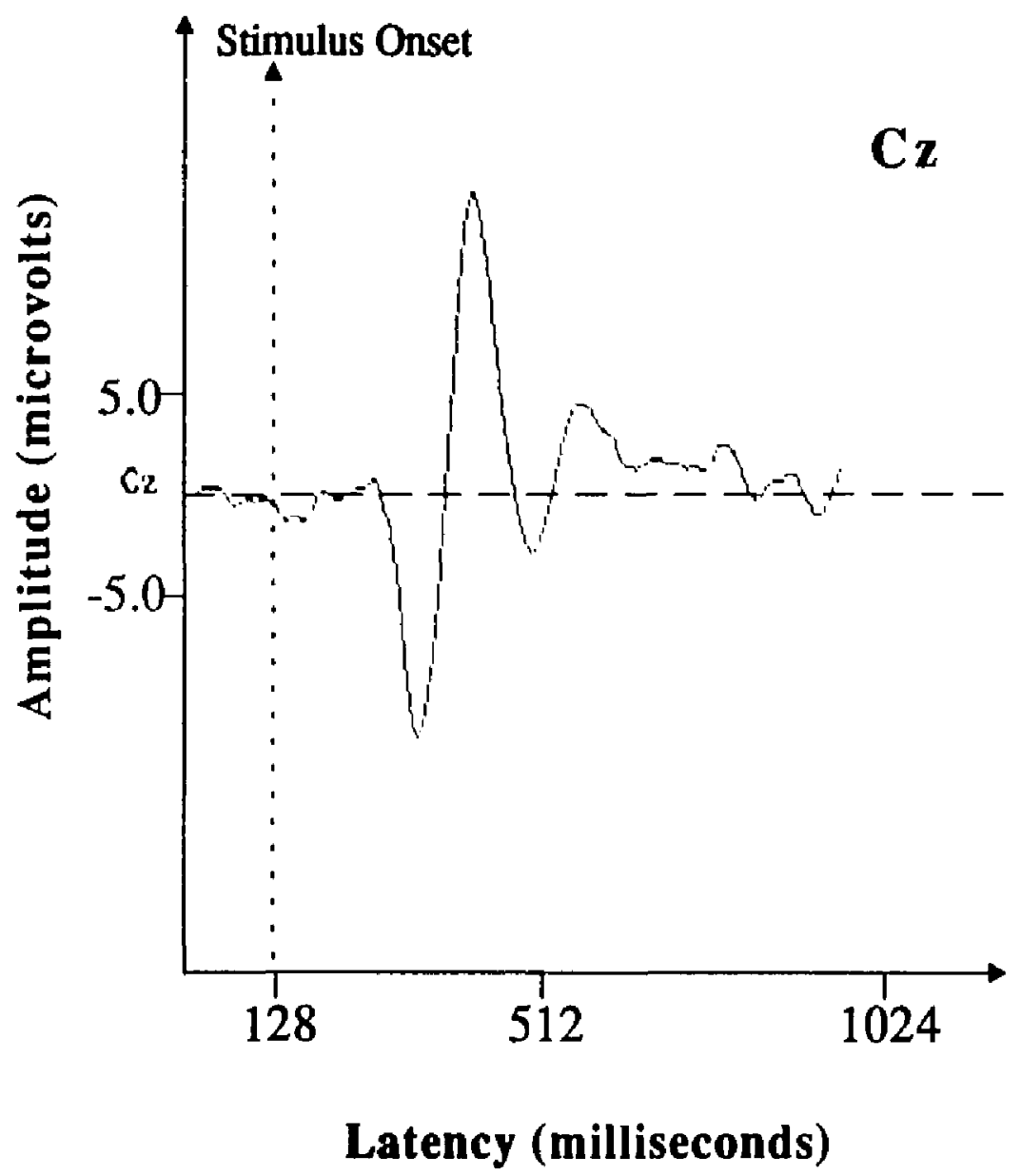

FIGURE 40. Grand average waveform across all subjects in Condition 1 (plain [da] vs. duplex [da]). This waveform represents only the responses to duplex [da]. 


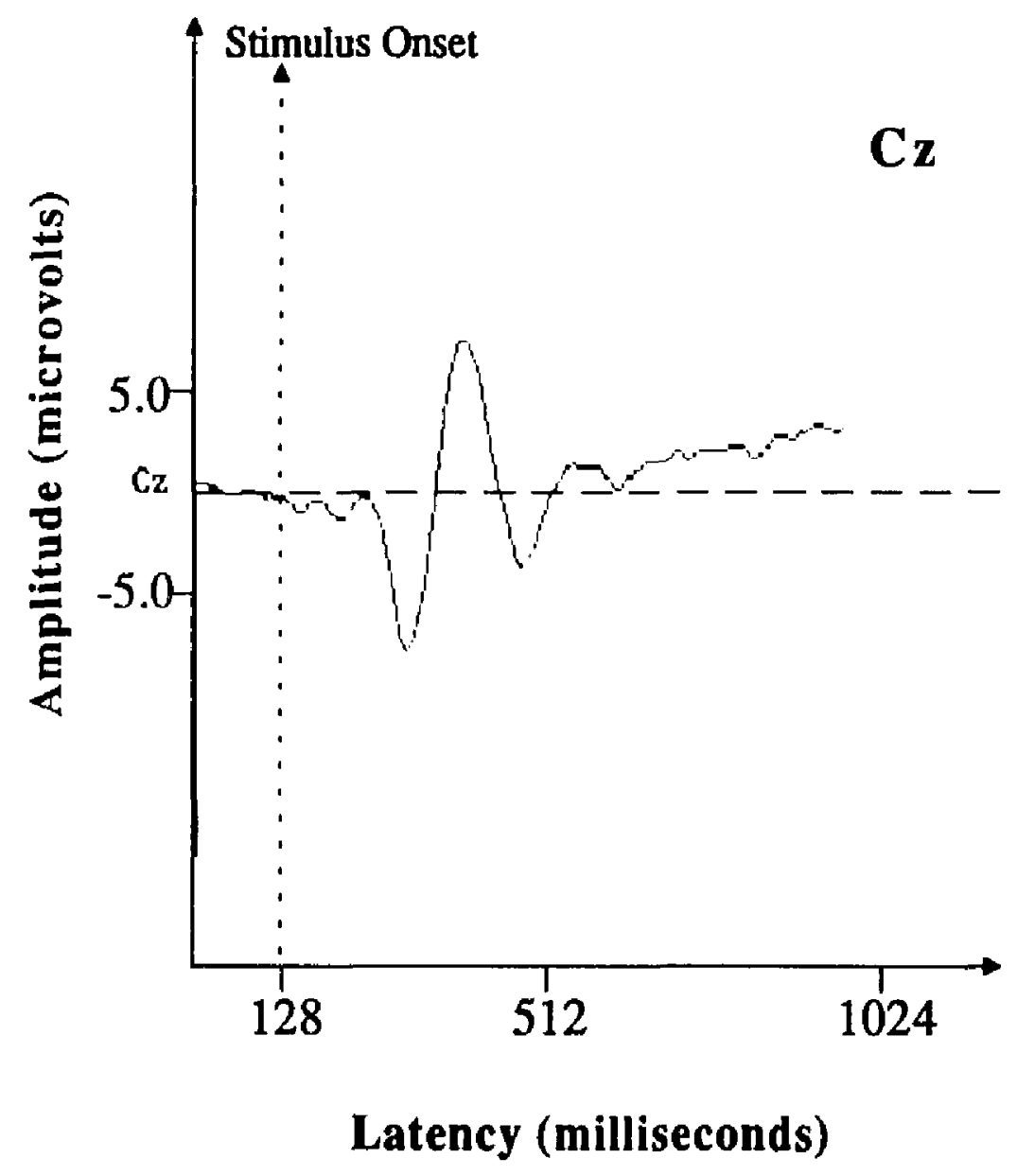

FIGURE 41. Grand average waveform across all subjects in Condition 2 (plain [ga] vs. duplex [ga]). This waveform represents only the responses to plain [ga]. 


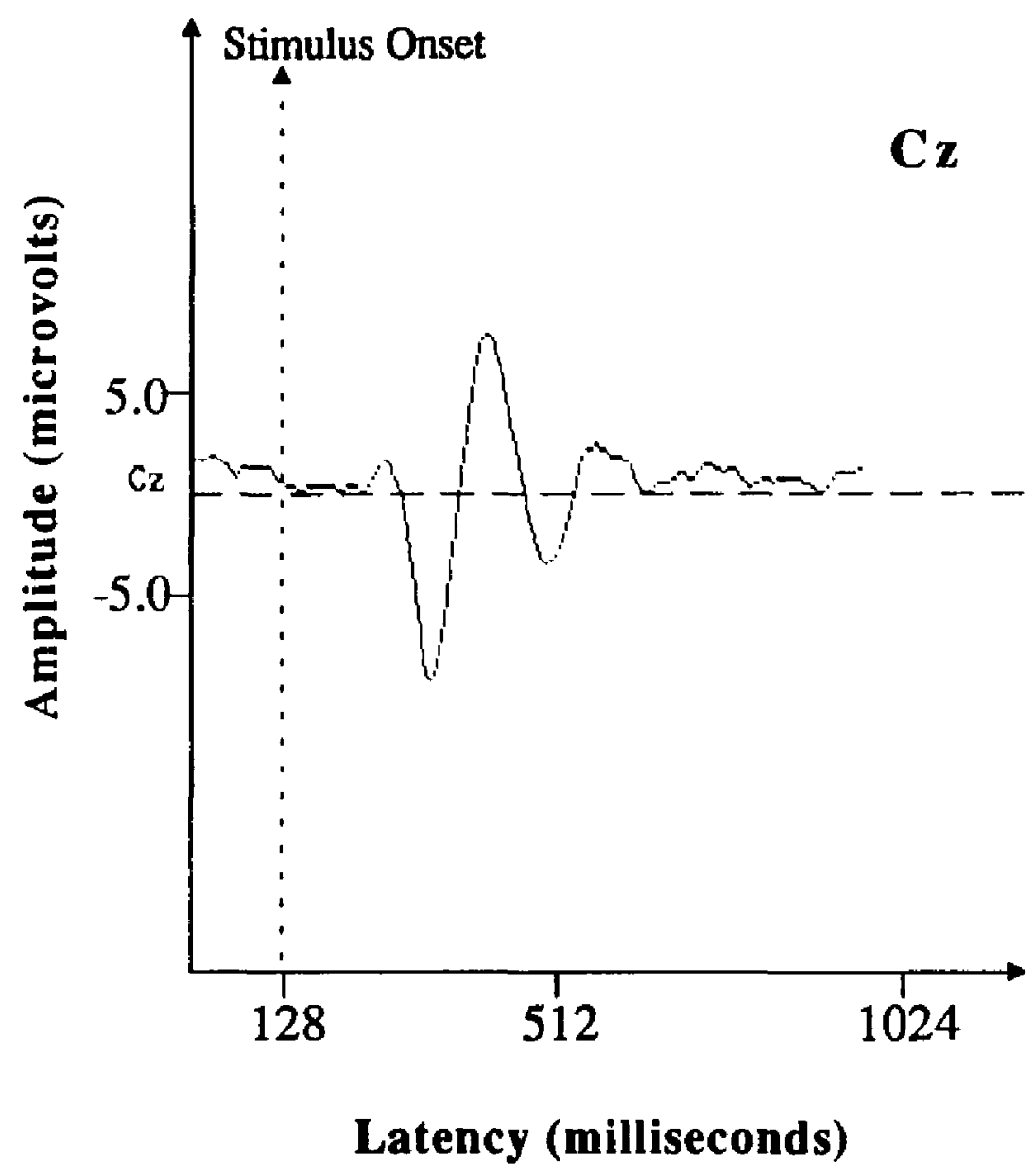

FIGURE 42. Grand average waveform across all subjects in Condition 2 (plain [ga] vs. duplex [ga]). This waveform represents only the responses to duplex [ga]. 


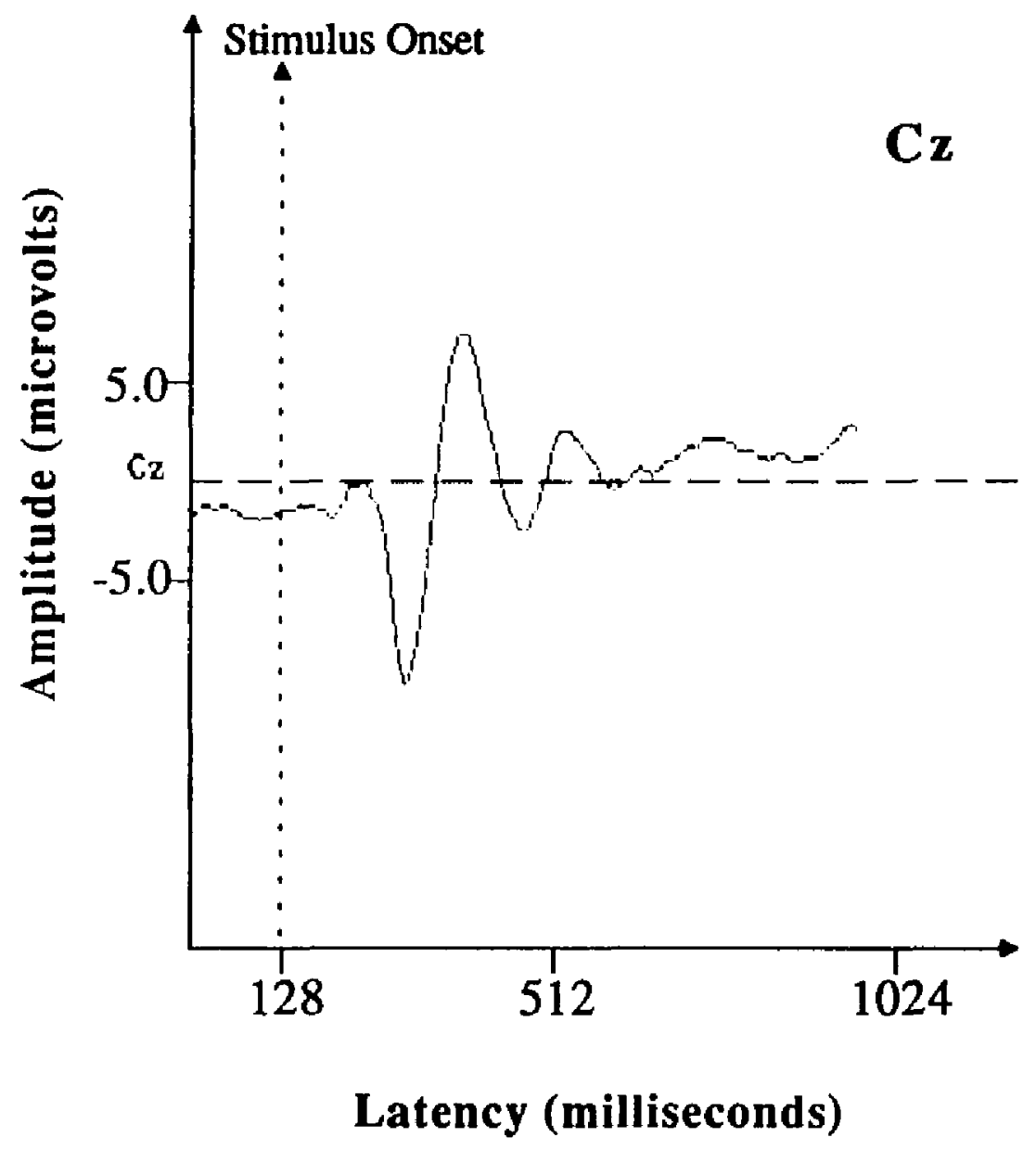

FIGURE 43. Grand average waveform scross all subjects in Condition 3 (duplex [da] vs. duplex [ga]). This waveform represents only the responses to duplex [da]. 


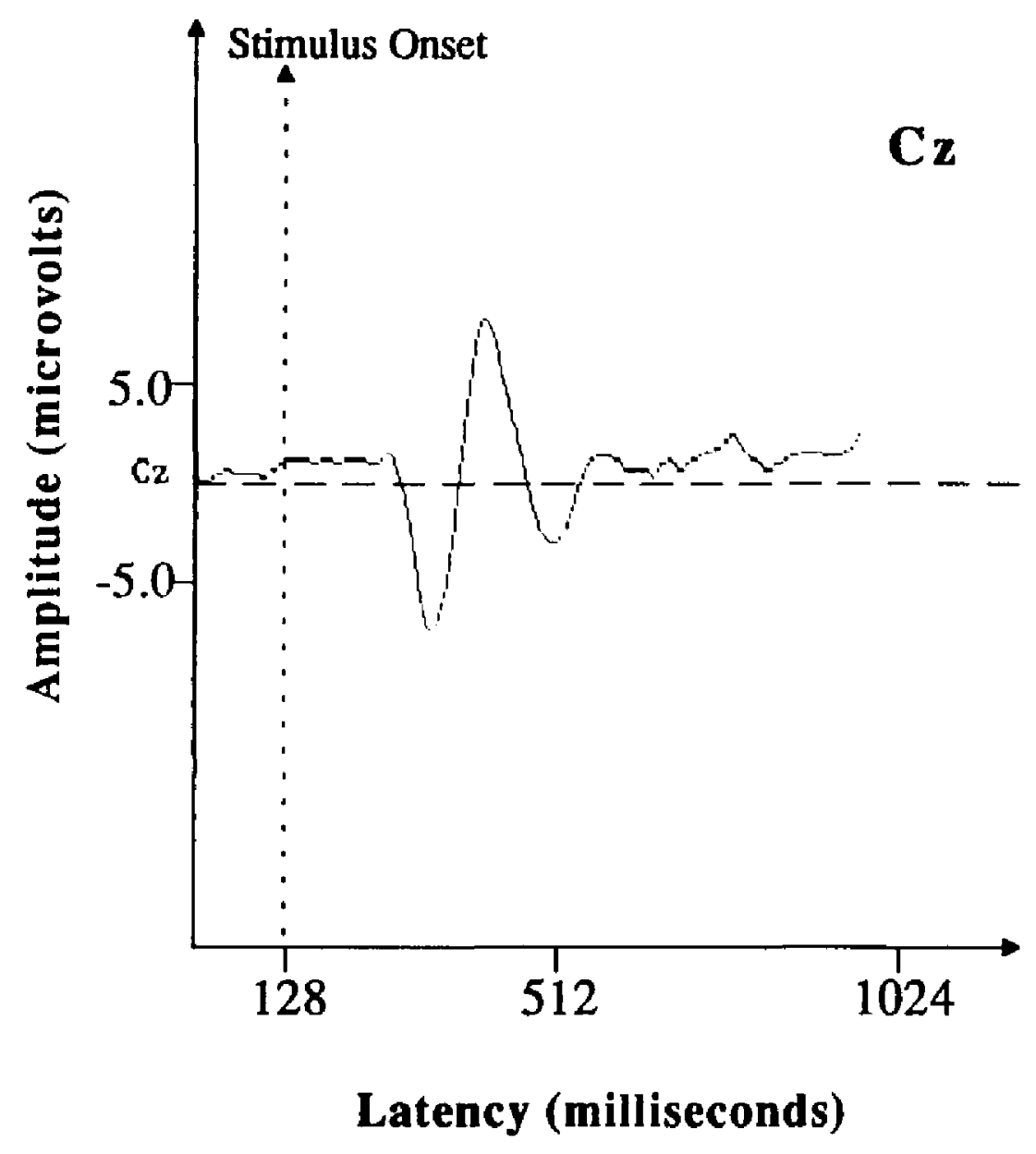

FIGURE 44. Grand average waveform across all subjects in Condition 3 (duplex [da] vs. duplex [ga]). This waveform represents only the responses to duplex [ga]. 


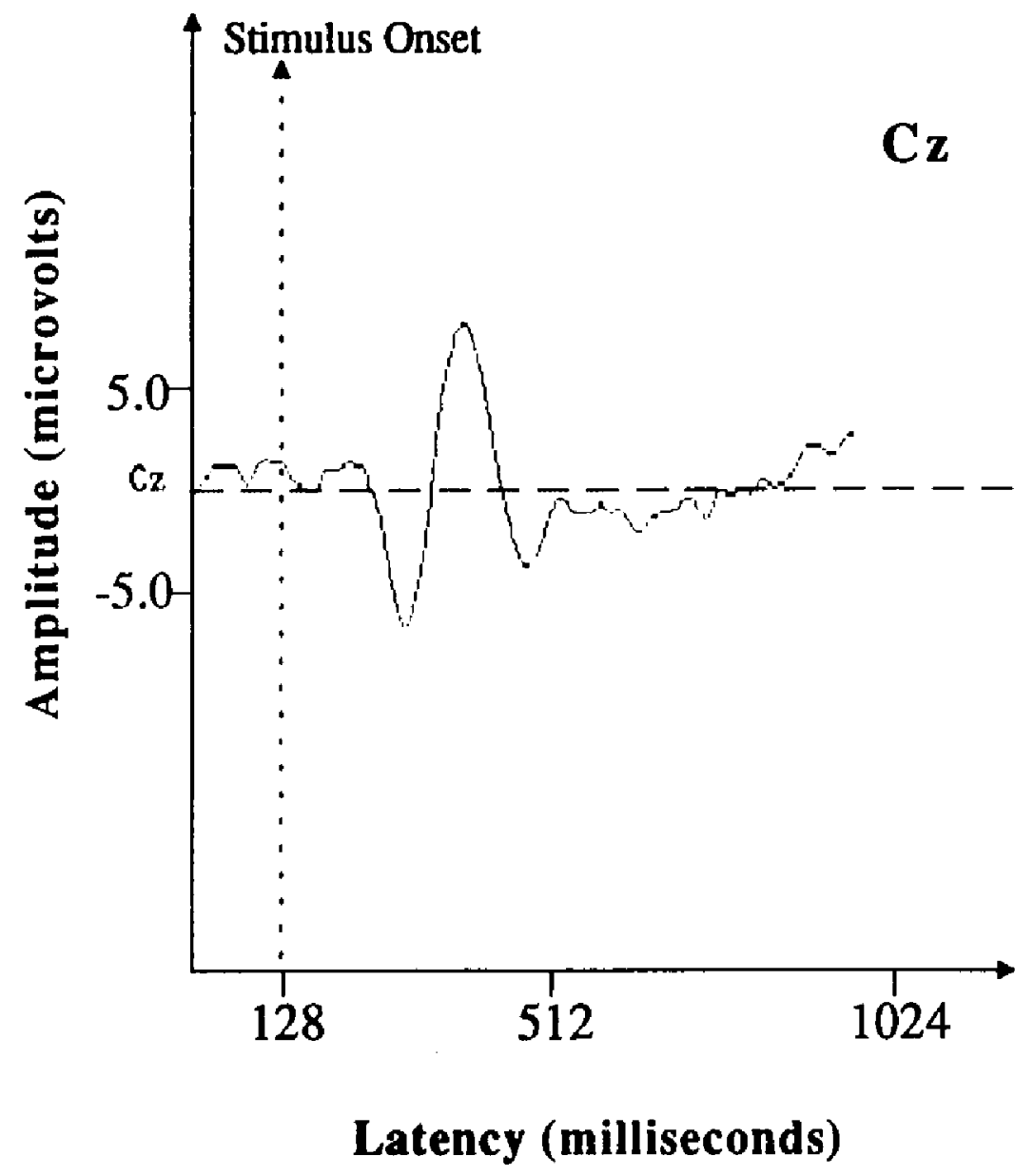

FIGURE 45. Grand average waveform across all subjects in Condition 4 (plain [da] vs. plain [ga]). This waveform represents only the responses to plain [da]. 


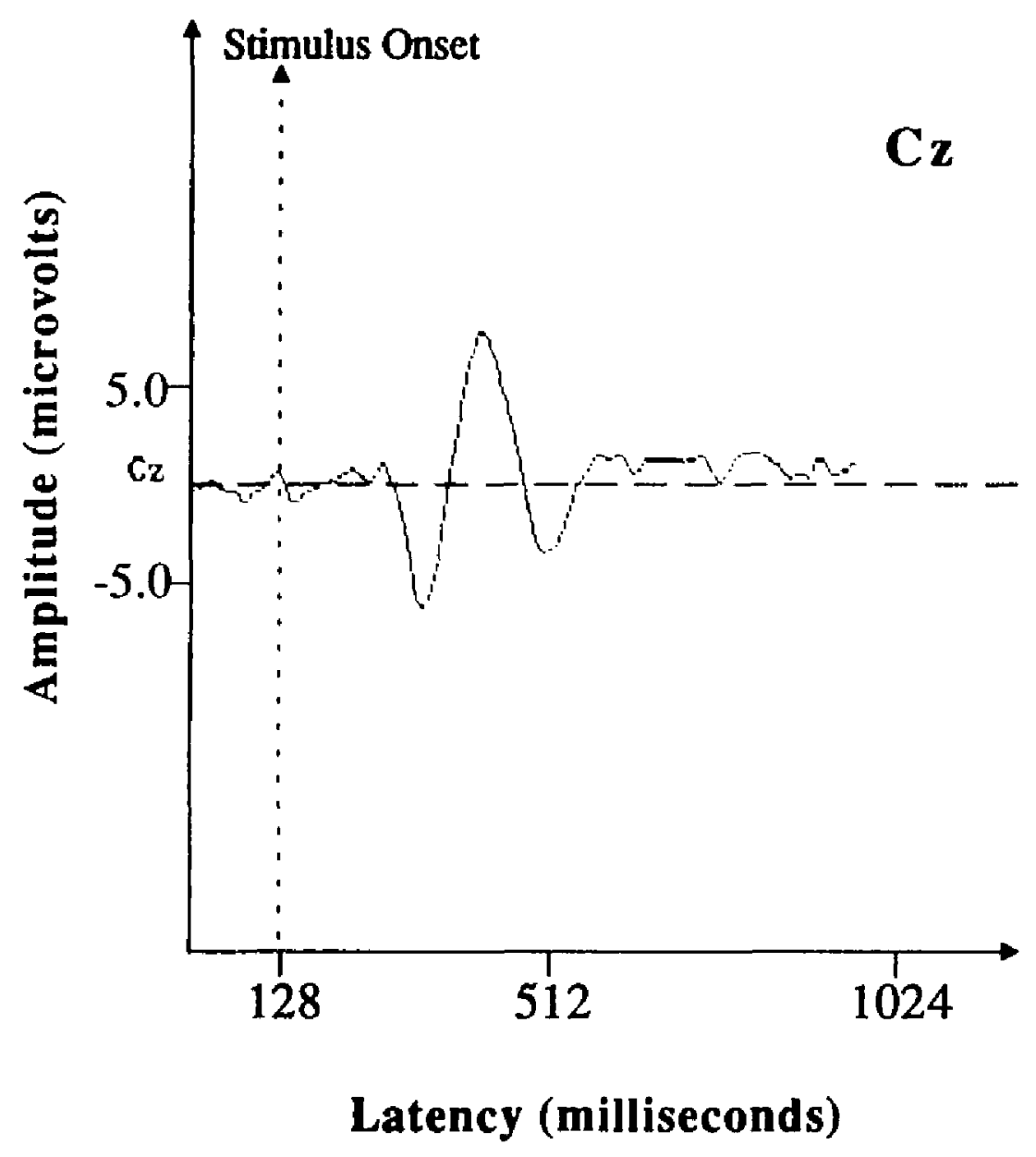

FIGURE 46. Grand average waveform across all subjects in Condition 4 (plain [da] vs. plain [ga]). This waveform represents only the responses to plain [ga]. 


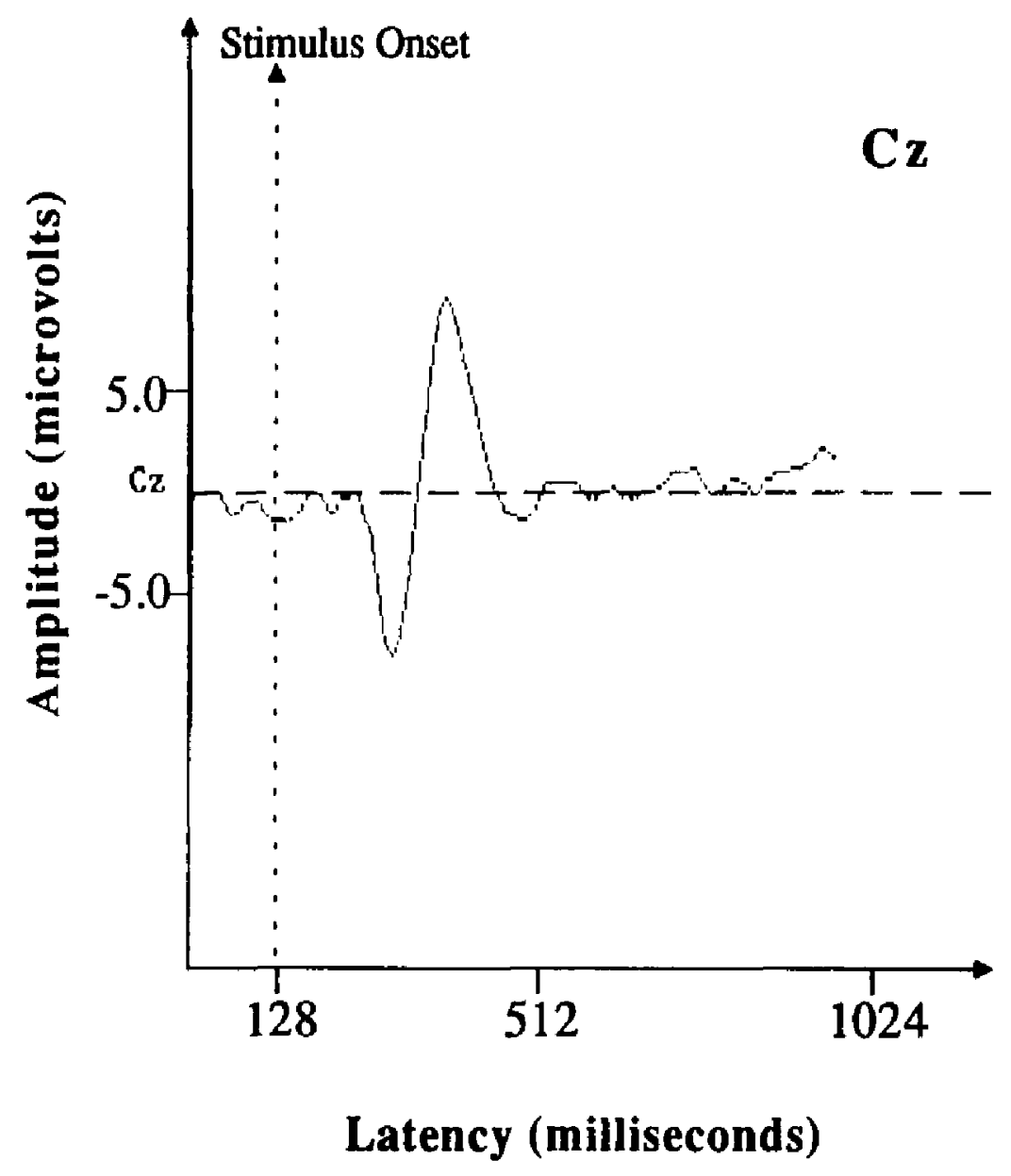

FIGURE 47. Grand average waveform across all subjects in Condition 5 (tone glide [da] vs. tone glide [ga]). This waveform represents only the responses to tone glide [da]. 


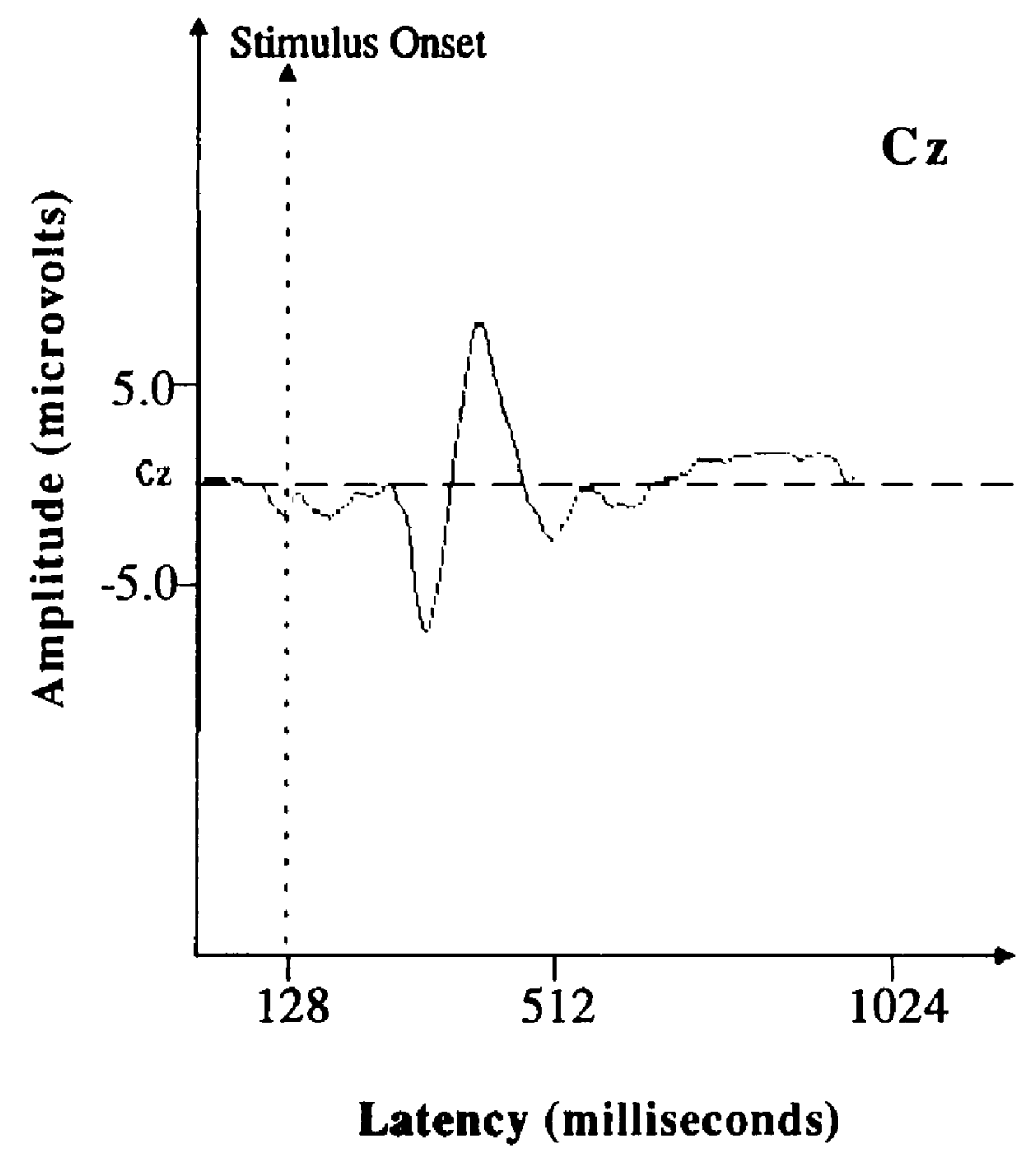

FIGURE 48. Grand average waveform across all subjects in Condition 5 (tone glide [da] vs. tone glide [ga]). This waveform represents only the responses to tone glide [ga]. 


\section{BIBLIOGRAPHY}

Boddy, J. and Weinberg, H. (1981). Brain potentials, perceptual mechanisms and semantic categorisation. Biological Psychology, 12, 43-61.

Browman, C. P., and Goldstein, L. M. (1985). Dynamic modeling of phonetic structure. In V. Fromkin (Ed.), Phonetic Linguistics. New York: Academic Press.

Chomsky, N., and Miller, G. A. (1963). Introduction to the formal analysis of natural languages. In R. D. Luce, R. Bush, and E. Galanter (Eds.), Handbook of mathematical psychology (Vol. 2). New York: Wiley.

Cohn, R. (1971). Differential cerebral processing of noise and verbal stimuli. Science, 172, 599-601.

Cole, R. A. and Scott, B. (1974). The phantom in the phoneme: Invariant cues for stop consonants. Perception and Psychophysics, 15, 101-107.

Cutting, J. E. (1974). Two left-hemisphere mechanisms in speech perception. Perception and Psychophysics, 16, 601-612.

Darwin, C. J. (1992). The relationship between speech perception and the perception of other sounds. In Mattingly and Studdert-Kennedy (Eds.), Modularity and the Motor Theory of Speech Perception (pp. 239-259). New Jersey: Lawrence Erlbaum Associates. 
Elman, J. L., and McClelland, J. L. (1984). Speech perception as a cognitive process: The interactive activation model. In N. Lass (Ed.),Language and Speech, 10 (pp. 337-374). New York: Academic Press.

Fant, G. (1967). Auditory patterns of speech. In W. Wathen-Dunn (Ed.), Models for the Perception of Speech and Visual Form. Cambridge, MA: MIT Press.

Fischler, I., Bloom, P. A., Childers, D. A., Roucos, S. E., and Perry, N. W. (1982). Potentials related to sentence verification: Lexical versus sentential processes and the N400. (In press).

Fleck, K. M. and Polich, J. (1988). P300 and the menstrual cycle. Electroencephalography and clinical Neurophysiology, 71, 157-160.

Fodor, J. (1983). The Modularity of Mind. Cambridge, MA: MIT Press.

Fowler, C. A. (1986). An event approach to the study of speech perception from a direct-realist perspective. Journal of Phonetics, 14, 3-28.

Fowler, C. A. (1989). Real objects of speech perception: A commentary on Diehl and Kluender. Biological Psychology, 1 (2), 145-160.

Fowler, C. A., and Rosenblum, L. D. (in press). Duplex perception: A comparison of monosyllables and slamming doors. Journal of Experimental Psychology: Human Perception and Performance.

Galambos, R., Benson, P., Smith, T. S., Schulman-Galambos, C., and Osier, H. (1975). On hemispheric differences in evoked potentials to speech stimuli. Electroencephalography and clinical Neurophysiology, 39, 279-283. 
Garnsey, S. M., Tanenhaus, M. K., and Chapman, R. M. (1989). Evoked potentials and the study of sentence comprehension. Journal of Psycholinguistic Research, 18 (1), 51-60.

Grabow, J. D., Aronson, A. E., Rose, D. E., and Greene, K. L. (1980). Summated potentials evoked by speech sounds for determining cerebral dominance for language. Electroencephalography and clinical Neurophysiology, 49, 38-47.

Greenberg, H. J. (1970). Electroencephalographic changes during learning of speech and nonspeech stimuli. Journal of Verbal Learning Behavior, 274-281.

Hillyard, S. A. and Woods, D. L. (1979). Electrophysiological analysis of human brain function. In Gazzaniga (Ed.), Handbook of Behavioral Neurology, Vol. 2. New York: Plenum Press.

Jasper, H. H. (1958). The ten-twenty electrode system of the International Federation. Electroencephalography and clinical Neurophysiology, $10,371-375$.

Johnston, V. S. and Wang, X. T. (1991). The relationship between menstrual phase and the P3 component of ERPs. Psychophysiology, $28,4,400-409$.

Julesz, B. (1960). Binocular depth perception of computer-generated patterns. Bell System Technical Journal, 39, 1125-1162.

Julesz, B. (1971). Foundations of Cyclopean Perception. Chicago: University of Chicago Press.

Kewley-Port, D. (1980). Representations of spectral change as cues to place of articulations in stop consonants. Research on speech perception (Technical Rep. No. 3). Bloomington, IN: Speech Research Laboratory. 
Kewley-Port, D. and Luce, P. A. (1984). Time-varying features of initial stop consonants in auditory running spectra: A first report. Perception and Psychophysics, 35, 353-360.

Kimura, D. (1964). Left-right differences in the perception of melodies. Quarterly Journal of Experimental Psychology, 16, 335-358.

Kimura, D. (1967). Functional asymmetry of the brain in dichotic listening.Cortex, 3, 163-178.

Klatt, D. H. (1978). Synthesis of consonant + vowel syllables. Unpublished manuscript, Massachusetts Institute of Technology.

Klatt, D. H. (1980a). Speech perception: A model of acoustic-phonetic analysis and lexical access. In R. A. Cole (Ed.), Perception and Production of Fluent Speech. Hillsdale, NJ: Erlbaum.

Klatt, D. H. (1980b). Software for a cascade/parallel formant synthesizer. Journal of the Acoustical Society of America, 67, 971-995.

Kluender, K. R. and Greenberg, S. (1989). A specialization for speech perception?. [Letter to the editor]. Science, 244, 1530-1531.

Kluender, K. R., Diehl, R. L., and Killeen, P. R. (1987). Japanese quail can learn phonetic categories. Science, 237, 1195-1197.

Kuriki, S. and Murase, M. (1989). Neuromagnetic study of the auditory responsed in right and left hemispheres of the human brain evoked by pure tones and speech sounds. Experiments in Brain Research, 77, 127-134.

Kutas, M. and Hillyard, S. A. (1988). An electrophysiological probe of incidental semantic association. Journal of Cognitive Neuroscience, $1(1), 38-49$. 
Kutas, M. and Hillyard, S. A. (1983). Event-related brain potentials to grammatical errors and semantic anomalies. Memory \& Cognition, $11(5), 539-550$.

Kutas, M. and Hillyard, S. A. (1980). Reading senseless sentences: Brain potentials reflect semantic incongruity. Science, 207 (11), 203-205.

Kutas, M., Van Petten, C., and Besson, M. (1988). Event-related potential asymmetries during the reading of sentences. Electroencephalography and clinical Neurophysiology, 69, 218-233.

Ladefoged, P. (1962). Elements of Acoustic Phonetics. Chicago: University of Chicago Press.

Liberman, A. M. (1979). Duplex perception and integration of cues: Evidence that speech is different from nonspeech and similar to language. In Fischer-Jorgenson, J. Rischel, and N. Thorsen (Eds.), Proceedings of the IXth International Congress of Phonetic Sciences. Copenhagen: University of Copenhagen.

Liberman, A. M. and Mattingly, I. G. (1989). A specialization for speech perception. Science, $243,489-494$.

Liberman, A. M. and Mattingly, I. G. (1985). The motor theory of speech perception revised. Cognition, 21, 1-36.

Liberman, A. M., Cooper, F. S., Shankweiler, D. P., and Studdert-Kennedy, M. (1967). Perception of the speech code. Psychological Review, 74, 431-461.

Liberman, A. M., Delattre, P. C., Cooper, F. C., and Gerstman, L. J. (1954). The role of consonant-vowel transitions in the perception of the stop and nasal consonants. Psychological Monographs, 68, 1-13. 
Luce, P. A. and Pisoni, D. B. (1987). Speech perception: New directions in research, theory, and applications. In $\mathbf{H}$. Winitz (Ed.), Human Communication and Its Disorders (pp. 1-77). Norwood, NJ: Ablex.

Mann, V. A. and Liberman, A. M. (1983). Some differences between phonetic and auditory modes of perception. Cognition, 14, 211-235.

Marler, P. (1970). Birdsong and speech development: Could there be parallels? American Scientist, 58, 669-673.

Marslen-Wilson, W. D. (1980). Speech understanding as a psychological process. In J. Simon (Ed.), Spoken Language Generation and Understanding (pp. 39-67). New York: Reidel.

Mattingly, I. G., Liberman, A. M., Syrdal, A. K., and Halwes, T. (1971). Discrimination in speech and nonspeech modes. Cognitive Psychology, 2, 131-157.

Mattingly, I. G. and Studdert-Kennedy, M. (Eds.). (1992). Modularity and the Motor Theory of SpeechPerception. New Jersey: Lawrence Erlbaum Associates.

Molfese, D. L. (1984). Left hemisphere sensitivity to consonant sounds not displayed by the right hemisphere: Electrophysiological correlates. Brain and Language, 22, 109-127.

Molfese, D. L. (1983). Event related potentials and language processes. In Gaillard and Ritter (Eds.), Tutorials in Event Related Potential Research: Endogenous Components (pp. 345-368), Amsterdam: North-Holland Publishing Co.

Molfese, D. L. (1978). Left and right hemisphere involvement in speech perception: Electrophysiological correlates. Perception and Psychophysics, 23 (3), 237-243. 
Molfese, D. L., Freeman, R. B., and Palermo, D. S. (1975). The ontogeny of brain lateralization for speech and nonspeech stimuli. Brain and Language, 2, 356-368.

Molfese, D. L. and Molfese, V. J. (1988). Right-hemisphere responses from preschool children to temporal cues to speech and nonspeech materials: Electrophysiological correlates. Brain and Language, 33, 245-259.

Molfese, D. L. and Schmidt, A. (1983). An auditory evoked potential study of consonant perception in different vowel environments. Brain and Language, 18, 57-70.

Neville, H. J. (1974). Electrographic correlates of lateral asymmetry in the processing of verbal and nonverbal auditory stimuli. Journal of Psycholinguistic Research, 3, 151-163.

Novak, G. P., Kurtzberg, D., Kreuzer, J. A., and Vaughan, H. G., Jr. (1989). Cortical responses to speech sounds and their formants in normal infants: maturational sequence and spatiotemporal analysis. Electroencephalography and clincial Neurophysiology, 73, 295-305.

Nunez, P. L. (1981). Electric Fields of the Brain: The Neurophysics of $E E G$. New York: Oxford University Press.

Nusbaum, H. C., Schwab, E. C., and Sawusch, J. R. (1983). The role of "chirp" identification in duplex perception. Perception and Psychophysics, 33 (4), 323-332.

Ojemann, G. A. (1983). Brain organization for language from the perspective of electrical stimulation mapping. The Behavioral and Brain Sciences, pp. 189-230. Cambridge University Press.

Oldfield, R. C. (1971). The assessment and analysis of handedness: The Edinburgh Inventory. Neuropsychologia, 9, 97-113. 
Pickett, J. M. (1980). The Sounds of Speech Communcation. Baltimore, MD: University Park Press.

Picton, T. W. and Stuss, D. T. (1984). Event-related potentials in the study of speech and language: A critical review. In Caplan, Lecours, and Smith (Eds.), Biological Perspectives on Language (pp. 303-360), Cambridge, MA: MIT Press.

Pisoni, D. B. and Luce, P. A. (1987). Acoustic-phonetic representations in word recognition. Cognition, 25, 21-52.

Pisoni, D. B. and Sawusch, J. R. (1975). Some stages of processing in speech perception. In Cohen and Nooteboom (Eds.), Structure andProcessing Speech Perception (pp. 16-35). Heidelberg: SpringerVerlag.

Poggio, G. F. (1984). Processing of stereoscopic information in primate visual cortex. In G. M. Edelman, W. E. Gall, and W. M. Cowan (Eds.), Dynamic Aspects of Neocortical Function, New York: Wiley.

Rand, T. C. (1974). Dichotic release from masking for speech. Journal of the Acoustical Society of America, 55, 678-680.

Ratliff, S. S. and Greenberg, H. J. (1972). The averaged encephalic response to linguistic and nonlinguistic auditory stimuli. Journal of Auditory Research, XII (I), 14-25.

Remez, R. E., Rubin, P. E., Pisoni, D. B., and Carrell, T. D. (1981). Speech perception without traditional speech cues. Science, 212, 947-950.

Repp, B. H., Milburn, C., and Ashkenas, J. (1983). Duplex perception: Confirmation of fusion. Perception and Psychophysics, 33 (4), 333-337. 
Roth, W. T., Koppel, B. S., and Bertozzi, P. E. (1570). The effect of attention on the averaged evoked response to speech sounds. Electroencephalography and clinical Neurophysiology, 29, 38-46.

Searle, C. L., Jacobson, J. F., and Rayment, S. G. (1979). Stop consonant discrimination based on human audition. Journal of the Acoustical Society of America, 65, 799-809.

Studdert-Kennedy, M. (1974). The perception of speech. In T. A. Sebeok (Ed.), Current Trends in Linguistics. The Hague: Mouton.

Stuss, D. T., Sarazin, F. F., Leech, E. E., and Picton, T. W. (1983). Eventrelated potentials during naming and mental rotation. Electroencephalography and clinical Neurophysiology, 56, 133-146.

Suga, N. (1984). The extent to which bisonar information is represented in the auditory cortex. In G. M. Edelman, W. E. Gall, and W. M. Cowan, (Eds.), Dynamic Aspects of Neocortical Function. New York: Wiley.

Thorpe, W. H. (1958). The leaming of song patterns by birds, with especial reference to the song of the chaffinch, Fringilla coelebs. Journal of the Acoustical Society of America, 100, 535-570.

Torello, M. W. and McCarley, R. W. (1986). The use of topographical mapping techniques in clinical studies in psychiatry. In F. H. Duffy (Ed.), Topographic mapping of the brain (pp. 383-389), Boston: Butterworth Publishers.

Tobin, H. (1968). Late responses to speech stimuli as demonstrated by electroencephalography utilizing a summing computer technique. Unpublished doctoral dissertation, Purdue University, Purdue, Indiana. 
Wall, L. G., Fox, R. A., Moenter, D., and Dalebout, S. D. (1991). Effect of speech distinctions and age differences on auditory event-related potentials. Journal of the American Academy of Audiology, 2, 237-245.

Whalen, D. H. and Liberman, A. M. (1987). Speech perception takes precedence over nonspeech perception. Science, 237, 169-171.

Wicklegren, W. A. (1969). Context-sensitive coding, associative memory, and serial order in (speech) behavior. Psychological Review, 76, $1-15$.

Zatorre, R. J., Evans, A. C., Meyer, E., and Gjedde, A. (1992). Lateralization of phonetic and pitch discrimination in speech processing. Science, 256, 846-849. 